\title{
All-organic Composites of Ferro- and Piezoelectric Phosphonium Salts for Mechanical Energy Harvesting Application
}

Thangavel Vijayakanth, † Farsa Ram,, ," Balu Praveenkumar, ${ }^{*,}{ }^{\perp}$ Kadhiravan Shanmuganathan, ${ }^{*}$, ," Ramamoorthy Boomishankar*,t,

†Department of Chemistry and $¥$ Centre for Energy Science, Indian Institute of Science Education and Research (IISER), Pune, Dr. Homi Bhabha Road, Pune - 411008, India

E-mail: boomi@iiserpune.ac.in

\$Polymer Science and Engineering Division and "Academy of Scientific and Innovative Research, CSIR-National Chemical Laboratory, Dr. Homi Bhabha Road, Pune - 411008, India

E-mail: k.shanmuganathan@ncl.res.in

${ }^{\perp}$ PZT Centre, Armament Research and Development Establishment, Dr. Homi Bhabha Road, Pune - 411021, India

E-mail: praveenkumar@arde.drdo.in

\section{Supporting Information}

\begin{tabular}{|c|c|c|}
\hline S.N. & Table of Contents & Page No. \\
\hline 1 & Experimental Section & $2-5$ \\
\hline 2 & X-ray Crystallographic Information & $6-11$ \\
\hline 3 & Characterization Data & $13-28$ \\
\hline 4 & Ferroelectric, Dielectric and Piezoelectric \\
studies & $29-33$ \\
\hline 5 & $\begin{array}{r}\text { Phosphonium salt-TPU polymer composites } \\
\text { and their studies }\end{array}$ & $34-46$ \\
\hline 6 & $\begin{array}{r}\text { Durability, fatigue test, capacitor charging and } \\
\text { viscoelastic properties Data }\end{array}$ & $47-48$ \\
\hline
\end{tabular}




\section{EXPERIMENTAL SECTION}

\section{Synthesis of organoamino phosphonium salts with tetrahedral anions}

\section{Binary salt 1: Triphenyl isopropylaminophosphonium tetrafluoroborate (TPAP·BF 4 )}

To a stirred solution of TPAP. $\mathrm{Br}(3 \mathrm{~g}, 0.0075 \mathrm{~mol})$ in methanol $(25 \mathrm{~mL}), \mathrm{NaBF}_{4}(1.24 \mathrm{~g}, 0.0113 \mathrm{~mol})$ in water $(10 \mathrm{~mL})$ was slowly added with the formation of $\mathrm{NaBr}$ precipitate. The reaction mixture was stirred at room temperature for a further period of 10-15 min. and filtered through celite. The obtained clear solution was left for the crystallization at room temperature. Colorless block-shaped crystals of TPAP.BF 4 were obtained after 5 days. Yield: $2.97 \mathrm{~g}(97 \%)$.

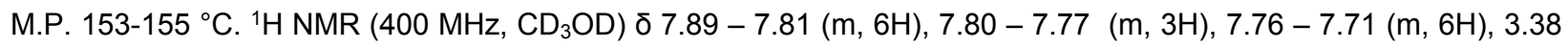
- $3.30(\mathrm{~m}, 1 \mathrm{H}), 1.19(\mathrm{~d}, J=6.4 \mathrm{~Hz}, 6 \mathrm{H}) .{ }^{13} \mathrm{C}$ NMR $\left(100 \mathrm{MHz}, \mathrm{CD}_{3} \mathrm{OD}\right) \delta 134.97,133.40,129.86,120.94,45.69$, 23.85. ${ }^{31} \mathrm{P}$ NMR (162 MHz, CD $\left.\mathrm{CD}_{3} \mathrm{OD}\right) \delta 36.55 .{ }^{19} \mathrm{~F} \mathrm{NMR}\left(377 \mathrm{MHz}, \mathrm{CD}_{3} \mathrm{OD}\right) \delta-154.70$ (s), -154.76 (s), -155.66 (dd, $J=$ 22.3, $11.1 \mathrm{~Hz}) .{ }^{11} \mathrm{~B}$ NMR $\left(128 \mathrm{MHz}, \mathrm{CD}_{3} \mathrm{OD}\right) \delta 0.43-0.17(\mathrm{~m}),-1.14(\mathrm{~s})$. FT-IR data in $\mathrm{KBr}$ pellet $\left(\mathrm{cm}^{-1}\right): 3211,2966$, 2879, 2815, 2734, 1587, 1462, 1370, 1113, 1083, 996, 749, 724 and 691. MALDI-TOF = 320.1563 [M+]. Anal. Calcd. For $\mathrm{C}_{21} \mathrm{H}_{23} \mathrm{BF}_{4} \mathrm{NP}$ : C 61.94; H 5.69; N 3.44. Found: C 61.91; H 5.63; N 3.43.

\section{Binary salt 2: Triphenyl isopropylaminophosphonium perchlorate (TPAP. $\mathrm{ClO}_{4}$ )}

To a stirred solution of TPAP. $\mathrm{Br}(3 \mathrm{~g}, 0.0075 \mathrm{~mol})$ in methanol $(25 \mathrm{~mL}), \mathrm{NaClO}_{4}(1.38 \mathrm{~g}, 0.0113 \mathrm{~mol})$ in water $(10 \mathrm{~mL})$ was slowly added with the formation of $\mathrm{NaBr}$ precipitate. The reaction mixture was stirred at room temperature for a further period of 10- $15 \mathrm{~min}$. and filtered through celite. The obtained clear solution was left for the crystallization at room temperature. Colorless block-shaped crystals of TPAP. $\mathrm{ClO}_{4}$ were obtained after one week. Yield: $3.09 \mathrm{~g}(98 \%)$. M.P. $155-158^{\circ} \mathrm{C} .{ }^{1} \mathrm{H}$ NMR $\left(400 \mathrm{MHz}, \mathrm{CD}_{3} \mathrm{OD}\right) \delta 7.89-7.81(\mathrm{~m}, 6 \mathrm{H}), 7.80-7.77$ (m, 3H), $7.76-7.71(\mathrm{~m}, 6 \mathrm{H}), 3.37$ - $3.31(\mathrm{~m}, 1 \mathrm{H}), 1.19(\mathrm{~d}, J=6.4 \mathrm{~Hz}, 6 \mathrm{H}) .{ }^{13} \mathrm{C} \mathrm{NMR}\left(100 \mathrm{MHz}, \mathrm{CD}_{3} \mathrm{OD}\right) \delta 135.03,133.48,129.94,121.00,45.76$, 23.89. ${ }^{31} \mathrm{P}$ NMR $\left(162 \mathrm{MHz}, \mathrm{CD}_{3} \mathrm{OD}\right) \delta$ 36.55. FT-IR data in $\mathrm{KBr}$ pellet $\left(\mathrm{cm}^{-1}\right): 3257,2970,2883,2360,1588,1484$, $1438,1391,1110,1041,997,903,751,725,692$ and 624. MALDI-TOF $=320.1563\left[\mathrm{M}^{+}\right]$. Anal. Calcd. For $\mathrm{C}_{21} \mathrm{H}_{23} \mathrm{CINO}_{4} \mathrm{P}: \mathrm{C} 60.08 ; \mathrm{H}$ 5.52; N 3.34. Found: C 60.02; H 5.53; N 3.31 .

\section{Binary salt 3: Triphenyl isopropylaminophosphonium periodate $\left(T P A P \cdot \mid \mathrm{O}_{4}\right)$}

To a stirred solution of TPAP. $\mathrm{Br}(1 \mathrm{~g}, 0.0025 \mathrm{~mol})$ in methanol $(25 \mathrm{~mL}), \mathrm{NalO}_{4}(0.81 \mathrm{~g}, 0.0038 \mathrm{~mol})$ in water $(10 \mathrm{~mL})$ was slowly added with the formation of $\mathrm{NaBr}$ precipitate. The reaction mixture was stirred at room temperature for a further period of 10-15 min. and filtered through celite. The obtained clear solution was left for the crystallization at room temperature. Colorless plate like crystals of TPAP. $1 \mathrm{O}_{4}$ were obtained after one week. Yield: $1.25 \mathrm{~g}(97 \%)$. M.P. $150-156{ }^{\circ} \mathrm{C} .{ }^{1} \mathrm{H}$ NMR $\left(400 \mathrm{MHz}, \mathrm{CD}_{3} \mathrm{OD}\right) \delta 7.90-7.81(\mathrm{~m}, 6 \mathrm{H}), 7.79-7.77(\mathrm{~m}, 3 \mathrm{H}), 7.75-7.73(\mathrm{~m}, 6 \mathrm{H}), 3.36-3.28$ (m, 1H), 1.19 (d, J = 6.4 Hz, 6H). ${ }^{13} \mathrm{C}$ NMR (100 MHz, CD $\left.{ }_{3} \mathrm{OD}\right) \delta$ 135.04, 133.49, 129.94, 121.01, 45.76, 23.90. ${ }^{11} \mathrm{P}$ NMR (162 MHz, $\left.\mathrm{CD}_{3} \mathrm{OD}\right) \delta$ 36.56. FT-IR data in $\mathrm{KBr}$ pellet $\left(\mathrm{cm}^{-1}\right): 3169,2967,2878,2815,1586,1484,1388,1176$, 1042, 996, 846, 755, 726 and 691. MALDI-TOF = $320.1563\left[\mathrm{M}^{+}\right]$. Anal. Calcd. For $\mathrm{C}_{21} \mathrm{H}_{23} \mathrm{INO}_{4} \mathrm{P}: \mathrm{C} 49.33 ; \mathrm{H} 4.53 ; \mathrm{N}$ 2.74. Found: C 49.31; H 4.51; N 2.75. 
<smiles>CC(C)NP(=O)(c1ccccc1)c1ccccc1</smiles>

TPAP.Br

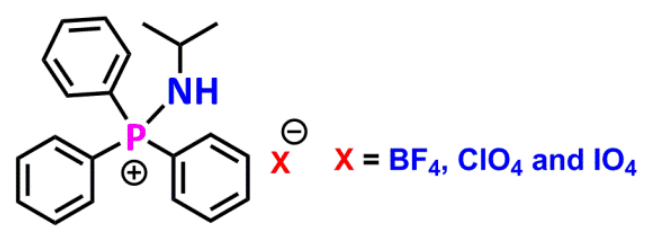

TPAP.X

Scheme S1. Synthesis of organic binary salts TPAP $X\left(X=\mathrm{BF}_{4}, \mathrm{ClO}_{4}\right.$ and $\left.\mathrm{IO}_{4}\right)$.

\section{Binary salt 4: Diphenyl diisopropylaminophosphonium tetrafluoroborate (DPDP·BF 4 )}

To a stirred solution of DPDP. $\mathrm{Br}(3 \mathrm{~g}, 0.0077 \mathrm{~mol})$ in methanol $(20 \mathrm{~mL}), \mathrm{NaBF}_{4}(1.27 \mathrm{~g}, 0.0116 \mathrm{~mol})$ in water $(10 \mathrm{~mL})$ was slowly added with the formation of $\mathrm{NaBr}$ precipitate. The reaction mixture was stirred at room temperature for a further period of 10-15 min. and filtered through celite. The obtained clear solution was left for the crystallization at room temperature. Colorless block-shaped crystals of $\mathrm{DPDP}^{\mathrm{B}} \mathrm{BF}_{4}$ were obtained after one week. Yield: $2.95 \mathrm{~g}(98 \%)$. M.P. $173-178{ }^{\circ} \mathrm{C} .{ }^{1} \mathrm{H}$ NMR $\left(400 \mathrm{MHz}, \mathrm{CD}_{3} \mathrm{OD}\right) \delta 7.87-7.80(\mathrm{~m}, 4 \mathrm{H}), 7.79-7.75(\mathrm{~m}, 2 \mathrm{H}), 7.68-7.63(\mathrm{~m}, 4 \mathrm{H}), 3.51-$ $3.39(\mathrm{~m}, 2 \mathrm{H}), 1.21(\mathrm{~d}, J=6.5 \mathrm{~Hz}, 12 \mathrm{H}) .{ }^{13} \mathrm{C}$ NMR $\left(100 \mathrm{MHz}, \mathrm{CD}_{3} \mathrm{OD}\right) \delta 134.36,132.41,129.46,124.58,43.90,23.65$. ${ }^{31} \mathrm{P}$ NMR (162 MHz, CD $\mathrm{OD}$ ) $\delta 33.20 .{ }^{19} \mathrm{~F}$ NMR (377 MHz, CD $3 \mathrm{OD}$ ) $\delta-154.55$ (s, $\left.{ }^{10} \mathrm{~B}\right),-154.60$ (s, $\left.{ }^{11} \mathrm{~B}\right),-155.53$ (dd, $J$ $=22.4,11.2 \mathrm{~Hz}) .{ }^{11} \mathrm{~B}$ NMR $\left(128 \mathrm{MHz}, \mathrm{CD}_{3} \mathrm{OD}\right) \delta$ 0.41-0.15 (m), -1.16 (s). FT-IR data in KBr pellet $\left(\mathrm{cm}^{-1}\right): 3297,3140$, 2975, 2835, 1590, 1464, 1389, 1127, 1055, 916, 890, 753, 726 and 696. MALDI-TOF = 301.1828 [M+]. Anal. Calcd. For $\mathrm{C}_{18} \mathrm{H}_{26} \mathrm{BF}_{4} \mathrm{~N}_{2} \mathrm{P}$ : C 55.69; H 6.75; N 7.22. Found: C 55.61; H 6.71; N 7.20.

\section{Binary salt 5: Diphenyl diisopropylaminophosphonium perchlorate (DPDP. $\mathrm{ClO}_{4}$ )}

To a stirred solution of DPDP. $\mathrm{Br}(3 \mathrm{~g}, 0.0077 \mathrm{~mol})$ in methanol $(20 \mathrm{~mL}), \mathrm{NaClO}_{4}(1.42 \mathrm{~g}, 0.0116 \mathrm{~mol})$ in water $(10 \mathrm{~mL})$ was slowly added with the formation of $\mathrm{NaBr}$ precipitate. The reaction mixture was stirred at room temperature for a further period of 10-15 min. and filtered through celite. The obtained clear solution was left for the crystallization at room temperature. Colorless block-shaped crystals of DPDP. $\mathrm{ClO}_{4}$ were obtained after one week. Yield: $3.03 \mathrm{~g}(98 \%)$. M.P. $190-195^{\circ} \mathrm{C} .{ }^{1} \mathrm{H}$ NMR $\left(400 \mathrm{MHz}, \mathrm{CD}_{3} \mathrm{OD}\right) \delta 7.88-7.81(\mathrm{~m}, 4 \mathrm{H}), 7.80-7.75(\mathrm{~m}, 2 \mathrm{H}), 7.68-7.63(\mathrm{~m}, 4 \mathrm{H}), 3.50-$ $3.41(\mathrm{~m}, 2 \mathrm{H}), 1.21(\mathrm{~d}, J=6.5 \mathrm{~Hz}, 12 \mathrm{H}) .{ }^{13} \mathrm{C}$ NMR $\left(100 \mathrm{MHz}, \mathrm{CD}_{3} \mathrm{OD}\right) \delta$ 134.42, 132.49, 129.52, 124.64, 43.97, 23.78. ${ }^{31} \mathrm{P}$ NMR $\left(162 \mathrm{MHz}, \mathrm{CD}_{3} \mathrm{OD}\right) \delta$ 33.23. FT-IR data in $\mathrm{KBr}$ pellet $\left(\mathrm{cm}^{-1}\right): 3263,2976,2872,1462,1426,1388,1117$, 1021, 997, 888, 832, 757, 725 and 695. MALDI-TOF $=301.1828\left[\mathrm{M}^{+}\right]$. Anal. Calcd. For $\mathrm{C}_{18} \mathrm{H}_{26} \mathrm{CIN}_{2} \mathrm{O}{ }_{4} \mathrm{P}: \mathrm{C} 53.94 ; \mathrm{H}$ 6.54; N 6.99. Found: C 53.91; H 6.52; N 6.95.

\section{Binary salt 6: Diphenyl diisopropylaminophosphonium periodate $\left(\mathrm{DPDP} \cdot 1 \mathrm{O}_{4} \mathrm{~L}_{\mathrm{L}}\right.$}

To a stirred solution of DPDP. $\mathrm{Br}(3 \mathrm{~g}, 0.0077 \mathrm{~mol})$ in methanol $(20 \mathrm{~mL}), \mathrm{NalO}_{4}(2.48 \mathrm{~g}, 0.0116 \mathrm{~mol})$ in water $(10 \mathrm{~mL})$ was slowly added with the formation of $\mathrm{NaBr}$ precipitate. The reaction mixture was stirred at room temperature for a further period of 10-15 min. and filtered through celite. The obtained clear solution was left for the crystallization at room temperature. Colorless block-shaped crystals of DPDP. $1 \mathrm{O}_{4}$ were obtained after one week. Yield: $3.65 \mathrm{~g} \mathrm{(96 \% ).}$ M.P. $142-145^{\circ} \mathrm{C} .{ }^{1} \mathrm{H}$ NMR $\left(400 \mathrm{MHz}, \mathrm{CD}_{3} \mathrm{OD}\right) \delta 7.88-7.82(\mathrm{~m}, 4 \mathrm{H}), 7.80-7.75(\mathrm{~m}, 2 \mathrm{H}), 7.68-7.64(\mathrm{~m}, 4 \mathrm{H}), 3.50-$ $3.41(\mathrm{~m}, 2 \mathrm{H}), 1.22(\mathrm{~d}, J=6.5 \mathrm{~Hz}, 12 \mathrm{H}) .{ }^{13} \mathrm{C}$ NMR $\left(100 \mathrm{MHz}, \mathrm{CD}_{3} \mathrm{OD}\right) \delta$ 134.44, 132.49, 129.53, 124.65, 43.96, 23.80. ${ }^{31} \mathrm{P}$ NMR (162 MHz, CD $\left.\mathrm{CD}_{3} \mathrm{OD}\right) \delta$ 33.23. FT-IR data in $\mathrm{KBr}$ pellet $\left(\mathrm{cm}^{-1}\right): 3141,2968,2931,2834,1426,1368,1161$, 1075, 1020, 845, 750 and 699. MALDI-TOF $=301.1828\left[\mathrm{M}^{+}\right]$. Anal. Calcd. For $\mathrm{C}_{18} \mathrm{H}_{26} \mathrm{IN}_{2} \mathrm{O}_{4} \mathrm{P}: \mathrm{C}$ 43.92; $\mathrm{H}$ 5.32; $\mathrm{N}$ 5.69. Found: C 43.90; H 5.31; N 5.68. 


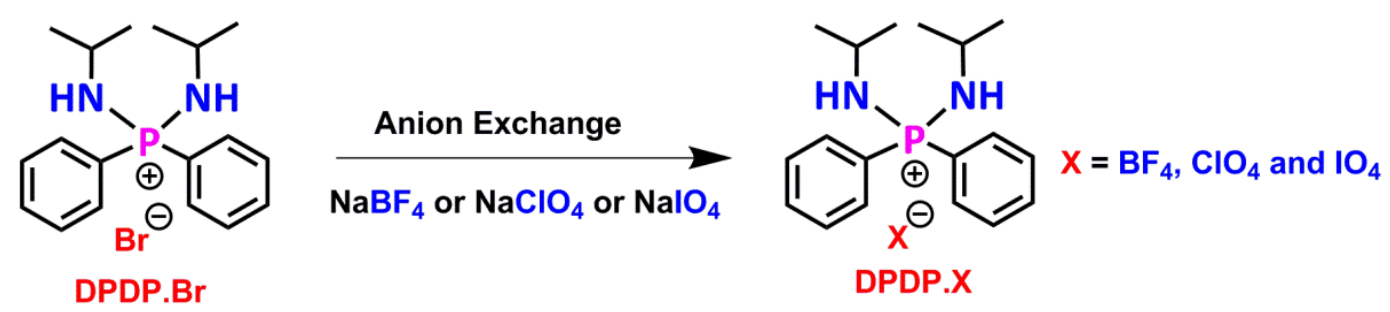

Scheme S2. Synthesis of organic binary salts DPDP.X $\left(X=\mathrm{BF}_{4}, \mathrm{ClO}_{4}\right.$ and $\left.\mathrm{IO}_{4}\right)$.

Binary salt 7: Phenyl triisopropylaminophosphonium tetrafluoroborate (PTAP.BF 4 )

To a stirred solution of PTAP.Br $(1 \mathrm{~g}, 0.0028 \mathrm{~mol})$ in methanol $(20 \mathrm{~mL}), \mathrm{NaBF}_{4}(0.46 \mathrm{~g}, 0.0042 \mathrm{~mol})$ in water (10 mL) was slowly added with the formation of $\mathrm{NaBr}$ precipitate. The reaction mixture was stirred at room temperature for a further period of 10-15 min. and filtered through celite. The obtained clear solution was left for the crystallization at room temperature. Colorless rod-shaped crystals of PTAP·BF 4 were obtained after one week. Yield: $0.98 \mathrm{~g}(98 \%)$. M.P. $146-149{ }^{\circ} \mathrm{C} .{ }^{1} \mathrm{H}$ NMR $\left(400 \mathrm{MHz}, \mathrm{CD}_{3} \mathrm{OD}\right) \delta 7.94-7.92(\mathrm{~m}, 1 \mathrm{H}), 7.91-7.88(\mathrm{~m}, 1 \mathrm{H}), 7.75-7.70(\mathrm{~m}, 1 \mathrm{H}), 7.66-$ $7.60(\mathrm{~m}, 2 \mathrm{H}), 3.34-3.32(\mathrm{~m}, 3 \mathrm{H}), 1.16(\mathrm{~d}, \mathrm{~J}=6.9 \mathrm{~Hz}, 18 \mathrm{H}) .{ }^{13} \mathrm{C}$ NMR $\left(100 \mathrm{MHz}, \mathrm{CD}_{3} \mathrm{OD}\right) \delta$ 133.70, 131.91, 129.05, 126.83, 43.25, 23.84. ${ }^{31} \mathrm{P}$ NMR (162 MHz, $\left.\mathrm{CD}_{3} \mathrm{OD}\right) \delta$ 29.89. ${ }^{19} \mathrm{~F}$ NMR (377 MHz, CD $\left.\mathrm{OD}\right) \delta-154.72\left(\mathrm{~s},{ }^{10} \mathrm{~B}\right),-154.77$ (s, ${ }^{11} \mathrm{~B}$ ), -155.67 (dd, $J=22.3,11.1 \mathrm{~Hz}$ ). ${ }^{11} \mathrm{~B}$ NMR (128 MHz, CD $\mathrm{OD}$ ) $\delta$ 0.45-0.19 (m), -1.13 (s). FT-IR data in KBr pellet $\left(\mathrm{cm}^{-1}\right)$ : 3138, 2975, 2936, 2879, 1591, 1468, 1422, 1124, 1033, 927, 882, 844, 750, 713 and 692. MALDI-TOF $=282.2094\left[\mathrm{M}^{+}\right]$. Anal. Calcd. For $\mathrm{C}_{15} \mathrm{H}_{29} \mathrm{BF}_{4} \mathrm{~N}_{3} \mathrm{P}: \mathrm{C} 48.80 ; \mathrm{H} 7.92 ; \mathrm{N} 11.38$. Found: C 48.71; H 7.91; N 11.37.

Binary salt 8: Phenyl triisopropylaminophosphonium perchlorate (PTAP.ClO 4

To a stirred solution of PTAP. Br $(3 \mathrm{~g}, 0.0083 \mathrm{~mol})$ in methanol $(20 \mathrm{~mL}), \mathrm{NaClO}_{4}(2.29 \mathrm{~g}, 0.0125 \mathrm{~mol})$ in water (10 $\mathrm{mL}$ ) was slowly added with the formation of $\mathrm{NaBr}$ precipitate. The reaction mixture was stirred at room temperature for a further period of 10-15 min. and filtered through celite. The obtained clear solution was left for the crystallization at room temperature. A mixture of crystals were identified and separated under the microscope. The plate-like crystals were used for X-ray diffraction analysis, which shows the presence of phosphonium cation and tetrafluoroborate anion, respectively. However, we were unable to separate more quantity of $\mathrm{PTAP} \cdot \mathrm{ClO}_{4}$ is due to poor quality of crystals and hence for other characterizations.

Binary salt 9: Phenyl triisopropylaminophosphonium periodate $\left(\mathrm{PTAP} \cdot \mathrm{IO}_{4}\right)$

To a stirred solution of PTAP. Br $(1 \mathrm{~g}, 0.0028 \mathrm{~mol})$ in methanol $(20 \mathrm{~mL}), \mathrm{NalO}_{4}(0.90 \mathrm{~g}, 0.0042 \mathrm{~mol})$ in water $(10 \mathrm{~mL})$ was slowly added with the formation of $\mathrm{NaBr}$ precipitate. The reaction mixture was stirred at room temperature for a further period of 10-15 min. and filtered through celite. The obtained clear solution was left for the crystallization at room temperature. Colorless rod-shaped crystals of PTAP $1 \mathrm{O}_{4}$ were obtained after one week. Yield: $1.28 \mathrm{~g}(96 \%)$. M.P. $153-156{ }^{\circ} \mathrm{C} .{ }^{1} \mathrm{H}$ NMR $\left(400 \mathrm{MHz}, \mathrm{CD}_{3} \mathrm{OD}\right) \delta 7.93-7.91(\mathrm{~m}, 1 \mathrm{H}), 7.91-7.88(\mathrm{~m}, 1 \mathrm{H}), 7.73-7.68(\mathrm{~m}, 1 \mathrm{H}), 7.65-$ $7.60(\mathrm{~m}, 2 \mathrm{H}), 3.33-3.32(\mathrm{~m}, 3 \mathrm{H}), 1.14(\mathrm{~d}, \mathrm{~J}=6.9 \mathrm{~Hz}, 18 \mathrm{H}) .{ }^{13} \mathrm{C}$ NMR $\left(100 \mathrm{MHz}, \mathrm{CD}_{3} \mathrm{OD}\right) \delta$ 133.71, 131.92, 129.03, 126.81, 43.27, 23.83. ${ }^{31} \mathrm{P}$ NMR (162 MHz, CD 3 OD) $\delta$ 29.91. FT-IR data in $\mathrm{KBr}$ pellet $\left(\mathrm{cm}^{-1}\right): 3217,2966,2869,1471$, 1434, 1187, 1045, 1012, 908, 877, 805, 748, 726 and 693. MALDI-TOF = 282.2094 [M+1. Anal. Calcd. For $\mathrm{C}_{15} \mathrm{H}_{29} \mathrm{IN}_{3} \mathrm{O}_{4} \mathrm{P}: \mathrm{C} 38.07$; $\mathrm{H}$ 6.18; N 8.88. Found: C 38.01; H 6.15; N 8.84. 


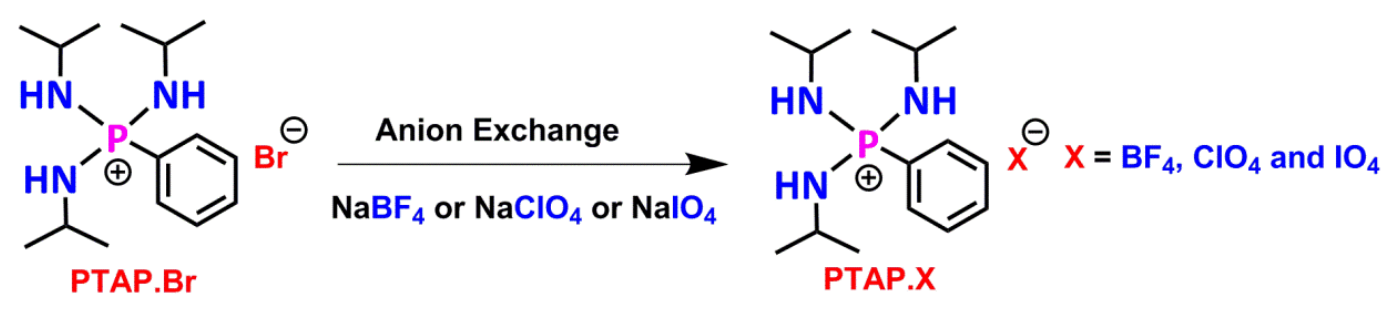

Scheme S3. Synthesis of organic binary salt PTAP.X $\left(X=\mathrm{BF}_{4}, \mathrm{ClO}_{4}\right.$ and $\left.\mathrm{IO}_{4}\right)$.

Tetraisopropylaminophosphonium chloride (TIAP.Cl)

The compound TIAP.Cl was synthesized by the modified literature procedure. ${ }^{1}$ To a solution of $\mathrm{PCl}_{5}(5 \mathrm{~g}, 0.024 \mathrm{~mol})$ in dry hexane $(100 \mathrm{~mL})$ kept at $-10^{\circ} \mathrm{C}$ under a nitrogen atmosphere, a freshly distilled isopropylamine $(11.35 \mathrm{~g}, 0.192$ mol) was added dropwise at $-10^{\circ} \mathrm{C}$. Further, the resulting reaction mixture was stirred at $-10^{\circ} \mathrm{C}$ under a nitrogen atmosphere for 30 minutes and then was slowly brought back to room temperature to yield a white colored solid. The final reaction mixture was then refluxed for a further period of 5 hours until the white precipitate formation is ceased. Distilled water $(100 \mathrm{~mL})$ was added to this reaction mixture and the water-insoluble product was isolated. Yield $6.86 \mathrm{~g}$

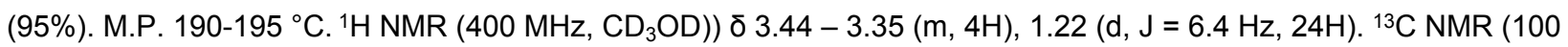
$\left.\mathrm{MHz}, \mathrm{CD}_{3} \mathrm{OD}\right)$ ) $\delta 43.45,24.01 .{ }^{31} \mathrm{P}$ NMR $\left(162 \mathrm{MHz}, \mathrm{CDCl}_{3}\right): \delta 20.01 \mathrm{ppm} . \mathrm{MALDI}-\mathrm{TOF}=263.2359$ [M+]. Anal. Calcd. For $\mathrm{C}_{12} \mathrm{H}_{32} \mathrm{CIN}_{4} \mathrm{P}$ : C 48.23; $\mathrm{H}$ 10.79; N 18.75. Found: C 48.17; H 10.23; N 18.12.

\section{Binary salt 10: Tetraisopropylaminophosphonium tetrafluoroborate $\left(\mathrm{TIAP} \cdot \mathrm{BF}_{4}\right)$}

To a stirred solution of TIAP.Cl $(3 \mathrm{~g}, 0.0100 \mathrm{~mol})$ in methanol $(20 \mathrm{~mL}), \mathrm{NaBF}_{4}(1.65 \mathrm{~g}, 0.015 \mathrm{~mol})$ in water $(10 \mathrm{~mL})$ was slowly added with the formation of $\mathrm{NaBr}$ precipitate. The reaction mixture was stirred at room temperature for a further period of 10-15 min. and filtered through celite. The obtained clear solution was left for the crystallization at room temperature. Colorless needle-shaped crystals of TIAP.BF ${ }_{4}$ were obtained after three days. Yield: $3.41 \mathrm{~g}(97 \%)$. M.P. $230-235{ }^{\circ} \mathrm{C} .{ }^{1} \mathrm{H}$ NMR $\left.\left(400 \mathrm{MHz}, \mathrm{CD}_{3} \mathrm{OD}\right)\right) \delta 3.41-3.32(\mathrm{~m}, 4 \mathrm{H}), 1.20(\mathrm{~d}, \mathrm{~J}=6.4 \mathrm{~Hz}, 24 \mathrm{H}) .{ }^{13} \mathrm{C} \mathrm{NMR}(100 \mathrm{MHz}$,

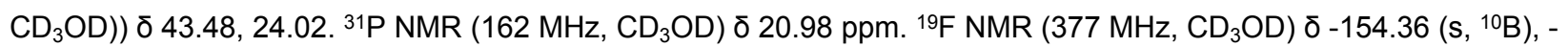
154.41 (s, ${ }^{11} \mathrm{~B}$ ), -155.35 (dd, $\mathrm{J}=22.4,11.1 \mathrm{~Hz}$ ). ${ }^{11} \mathrm{~B}$ NMR $\left(128 \mathrm{MHz}, \mathrm{CD}_{3} \mathrm{OD}\right) \delta$ 0.43-0.17 (m), -1.15 (s). FT-IR data in $\mathrm{KBr}$ pellet $\left(\mathrm{cm}^{-1}\right): 3220,2983,2932,2878,1425,1385,1367,1083,1036,919,896,731$ and 691. MALDI-TOF $=$ $263.2359\left[\mathrm{M}^{+}\right]$. Anal. Calcd. For $\mathrm{C}_{12} \mathrm{H}_{32} \mathrm{BF}_{4} \mathrm{~N}_{4} \mathrm{P}$ : C 41.16; $\mathrm{H}$ 9.21; N 16.00. Found: C 41.11; H 9.17; N 15.97.

\section{Binary salt 11: Tetraisopropylaminophosphonium perchlorate $\left(\mathrm{TIAP} \cdot \mathrm{ClO}_{4}\right)$}

To a stirred solution of TIAP.Cl $(3 \mathrm{~g}, 0.0100 \mathrm{~mol})$ in methanol $(20 \mathrm{~mL}), \mathrm{NaClO}_{4}(1.84 \mathrm{~g}, 0.015 \mathrm{~mol})$ in water $(10 \mathrm{~mL})$ was slowly added with the formation of $\mathrm{NaBr}$ precipitate. The reaction mixture was stirred at room temperature for a further period of 10-15 min. and filtered through celite. The obtained clear solution was left for the crystallization at room temperature. Colorless needle-shaped crystals of TIAP. $\mathrm{ClO}_{4}$ were obtained after one week. Yield: $3.53 \mathrm{~g}$ (97\%). M.P. $227-231{ }^{\circ} \mathrm{C} .{ }^{1} \mathrm{H}$ NMR $\left(400 \mathrm{MHz}, \mathrm{CD}_{3} \mathrm{OD}\right) \delta 3.42-3.32(\mathrm{~m}, 4 \mathrm{H}), 1.20$ (d, J = 6.4 Hz, 24H). ${ }^{13} \mathrm{C}$ NMR $(100$ $\left.\mathrm{MHz}, \mathrm{CD}_{3} \mathrm{OD}\right) \delta 43.56,24.10 .{ }^{31} \mathrm{P} \mathrm{NMR}\left(162 \mathrm{MHz}, \mathrm{CD}_{3} \mathrm{OD}\right) \delta 20.98 \mathrm{ppm}$. FT-IR data in KBr pellet $\left(\mathrm{cm}^{-1}\right): 3220,2972$, 2937, 2876, 1463 1423, 1385, 1120, 1094, 1037, 932, 897, 847, 726 and 626. MALDI-TOF = 263.2359 [M+]. Anal. Calcd. For $\mathrm{C}_{12} \mathrm{H}_{32} \mathrm{ClN}_{4} \mathrm{O}_{4} \mathrm{P}$ : C 39.72; $\mathrm{H} 8.89 ; \mathrm{N}$ 15.44. Found: C 39.73; $\mathrm{H} 8.87 ; \mathrm{N} 15.41$. 
Binary salt 12: Tetraisopropylaminophosphonium periodate $\left(\mathrm{TIAP} \cdot \mathrm{IO}_{4}\right)$

To a stirred solution of TIAP.Cl $(1 \mathrm{~g}, 0.0033 \mathrm{~mol})$ in methanol $(20 \mathrm{~mL}), \mathrm{NalO}_{4}(1.05 \mathrm{~g}, 0.0049 \mathrm{~mol})$ in water $(10 \mathrm{~mL})$ was slowly added with the formation of $\mathrm{NaBr}$ precipitate. The reaction mixture was stirred at room temperature for a further period of 10- $15 \mathrm{~min}$. and filtered through celite. The obtained clear solution was left for the crystallization process. Colorless block-shaped crystals of TIAP. $\mathrm{IO}_{4}$ were obtained after one week. Yield: $1.45 \mathrm{~g}(96 \%)$. M.P. 160$166{ }^{\circ} \mathrm{C} .{ }^{1} \mathrm{H}$ NMR $\left.\left(400 \mathrm{MHz}, \mathrm{CD}_{3} \mathrm{OD}\right)\right) \delta 3.41-3.32(\mathrm{~m}, 4 \mathrm{H}), 1.21(\mathrm{~d}, \mathrm{~J}=6.4 \mathrm{~Hz}, 24 \mathrm{H}) .{ }^{13} \mathrm{C}$ NMR $\left(100 \mathrm{MHz}, \mathrm{CD}_{3} \mathrm{OD}\right) \delta$ 43.56, 24.12. ${ }^{31} \mathrm{P}$ NMR $\left(162 \mathrm{MHz}, \mathrm{CD}_{3} \mathrm{OD}\right) \delta 20.99 \mathrm{ppm}$. FT-IR data in $\mathrm{KBr}$ pellet $\left(\mathrm{cm}^{-1}\right): 3218,2983,2966,1462$, $1383,1166,1123,1036,919,897,757,726$ and 619. MALDI-TOF $=263.2359\left[\mathrm{M}^{+}\right]$. Anal. Calcd. For $\mathrm{C}_{12} \mathrm{H}_{32} \mathrm{IN}_{4} \mathrm{O}_{4} \mathrm{P}$ : C 31.73; H 7.10; N 12.33. Found: C 31.67; H 7.11; N 12.35.

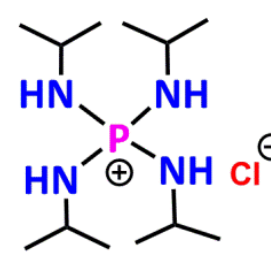

TIAP.CI

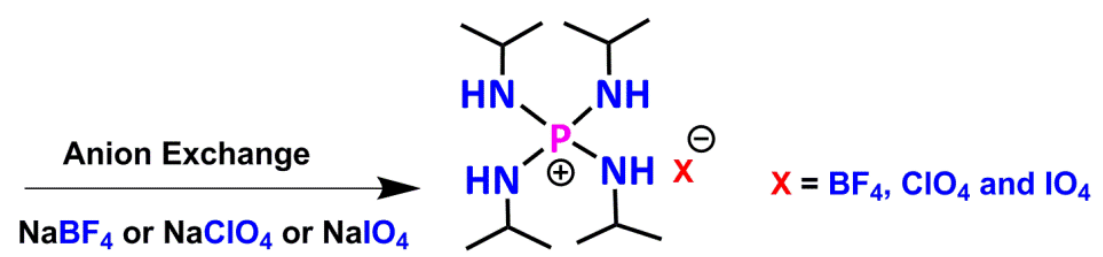

TIAP.X

Scheme S4. Synthesis of organic binary salt TIAP $X\left(X=\mathrm{BF}_{4}, \mathrm{ClO}_{4}\right.$ and $\left.\mathrm{IO}_{4}\right)$.

Table S1. Crystallographic data for all newly synthesized phosphonium salts at various temperatures.

\begin{tabular}{|c|c|c|c|c|}
\hline Compound & TPAP·BF ${ }_{4}$ & DPDP·BF 4 & DPDP·BF 4 & PTAP·BF 4 \\
\hline Chemical formula & $\mathrm{C}_{21} \mathrm{H}_{23} \mathrm{BF}_{4} \mathrm{NP}$ & $\mathrm{C}_{18} \mathrm{H}_{26} \mathrm{BF}_{4} \mathrm{~N}_{2} \mathrm{P}$ & $\mathrm{C}_{18} \mathrm{H}_{26} \mathrm{BF}_{4} \mathrm{~N}_{2} \mathrm{P}$ & $\mathrm{C}_{15} \mathrm{H}_{29} \mathrm{BF}_{4} \mathrm{~N}_{3} \mathrm{P}$ \\
\hline Formula weight & 407.18 & 388.19 & 388.19 & 369.19 \\
\hline Temperature & $100(2) \mathrm{K}$ & $100(2) \mathrm{K}$ & $150(2) \mathrm{K}$ & $100(2) \mathrm{K}$ \\
\hline Crystal system & Monoclinic & Monoclinic & Monoclinic & Monoclinic \\
\hline Space group & $P n$ & $P n$ & Cc & $P 2_{1} / n$ \\
\hline $\mathrm{a}(\AA) ; \alpha\left(^{\circ}\right)$ & $9.248(3) ; 90$ & 14.622(8); 90 & $15.5655(15) ; 90$ & $9.7284(12) ; 90$ \\
\hline $\mathrm{b}(\AA) ; \beta\left(^{\circ}\right)$ & $8.694(3) ; 104.641(7)$ & $9.048(5) ; 103.163(14)$ & $9.0923(9) ; 103.175(2)$ & $16.1549(19) ; 92.182(3)$ \\
\hline$c(\AA) ; \gamma\left({ }^{\circ}\right)$ & $12.874(4) ; 90$ & $15.561(8) ; 90$ & $14.6950(14) ; 90$ & $12.5433(14) ; 90$ \\
\hline $\mathrm{V}\left(\AA^{3}\right) ; \mathrm{Z}$ & 1001.5(6); 2 & $2004.6(18) ; 4$ & $2025.0(3) ; 4$ & $1969.9(4) ; 4$ \\
\hline$\rho$ (calc.) $\mathrm{g} \mathrm{cm}^{-3}$ & 1.350 & 1.286 & 1.273 & 1.245 \\
\hline$\mu\left(\mathrm{Mo} \mathrm{K}_{\alpha}\right) \mathrm{mm}^{-1}$ & 0.179 & 0.177 & 0.175 & 0.177 \\
\hline $2 \theta_{\max }\left({ }^{\circ}\right)$ & 54.66 & 52.74 & 56.54 & 55.76 \\
\hline $\mathrm{R}$ (int) & 0.0494 & 0.0959 & 0.0357 & 0.0630 \\
\hline Completeness to $\theta$ & 99.9 & 99.9 & 100 & 100 \\
\hline Data / param. & $3958 / 217$ & $7869 / 469$ & $4449 / 211$ & $4697 / 205$ \\
\hline GOF & 1.031 & 1.020 & 1.043 & 1.050 \\
\hline $\mathrm{R} 1[\mathrm{~F}>4 \sigma(\mathrm{F})]$ & 0.0403 & 0.0631 & 0.0559 & 0.0414 \\
\hline wR2 (all data) & 0.0937 & 0.1668 & 0.1608 & 0.1109 \\
\hline max. peak/hole $\left(e . \AA^{-3}\right)$ & $0.328 /-0.300$ & $1.473 /-0.513$ & $1.141 /-0.445$ & $0.593 /-0.622$ \\
\hline
\end{tabular}




\begin{tabular}{|c|c|c|c|c|}
\hline Compound & TIAP·BF 4 & TPAP. $\mathrm{ClO}_{4}$ & TPAP. $\mathrm{ClO}_{4}$ & DPDP.ClO ${ }_{4}$ \\
\hline Chemical formula & $\mathrm{C}_{12} \mathrm{H}_{32} \mathrm{BF}_{4} \mathrm{~N}_{4} \mathrm{P}$ & $\mathrm{C}_{21} \mathrm{H}_{23} \mathrm{ClNO}_{4} \mathrm{P}$ & $\mathrm{C}_{21} \mathrm{H}_{22} \mathrm{ClNO}_{4} \mathrm{P}$ & $\mathrm{C}_{18} \mathrm{H}_{26} \mathrm{CIN}_{2} \mathrm{O}_{4} \mathrm{P}$ \\
\hline Formula weight & 350.19 & 419.82 & 418.81 & 400.83 \\
\hline Temperature & $100(2) \mathrm{K}$ & $150(2) \mathrm{K}$ & $200(2) \mathrm{K}$ & $100(2) \mathrm{K}$ \\
\hline Crystal system & Tetragonal & Monoclinic & Monoclinic & Monoclinic \\
\hline Space group & $I-42 m$ & $P c$ & $P n$ & $P n$ \\
\hline$a(\AA) ; \alpha\left(^{\circ}\right)$ & $9.871(3) ; 90$ & $13.953(5) ; 90$ & $9.4106(19) ; 90$ & $14.6743(9) ; 90$ \\
\hline$b(\AA) ; \beta\left(^{\circ}\right)$ & $9.871(3) ; 90$ & $8.682(3) ; 107.560(9)$ & $8.751(19) ; 104.366(6)$ & $9.0435(6) ; 102.926(2)$ \\
\hline$c(\AA) ; \gamma\left({ }^{\circ}\right)$ & 9.547(3); 90 & $17.573(6) ; 90$ & 12.871(3); 90 & 15.6533(10); 90 \\
\hline $\mathrm{V}\left(\AA^{3}\right) ; \mathrm{Z}$ & $930.2(7) ; 2$ & $2029.6(11) ; 4$ & 1026.8(4); 2 & $2024.7(2) ; 4$ \\
\hline$\rho$ (calc.) $\mathrm{g} \mathrm{cm}^{-3}$ & 1.250 & 1.374 & 1.355 & 1.315 \\
\hline$\mu\left(\mathrm{Mo} \mathrm{K}_{\alpha}\right) \mathrm{mm}^{-1}$ & 0.185 & 0.294 & 0.291 & 0.292 \\
\hline $2 \theta_{\max }\left({ }^{\circ}\right)$ & 56.70 & 54.2 & 55.5 & 55.76 \\
\hline $\mathrm{R}$ (int) & 0.0419 & 0.1178 & 0.0767 & 0.0733 \\
\hline Completeness to $\theta$ & 100 & 99.9 & 100 & 100 \\
\hline Data / param. & $631 / 32$ & $7788 / 505$ & $4218 / 253$ & $9677 / 470$ \\
\hline GOF & 1.121 & 1.045 & 1.035 & 1.045 \\
\hline $\mathrm{R} 1[\mathrm{~F}>4 \sigma(\mathrm{F})]$ & 0.0221 & 0.0644 & 0.0570 & 0.0450 \\
\hline wR2 (all data) & 0.0577 & 0.1124 & 0.1337 & 0.1067 \\
\hline max. peak/hole $\left(e . \AA^{-3}\right)$ & $0.200 /-0.174$ & $0.379 /-0.361$ & $0.331 /-0.352$ & $0.982 /-0.740$ \\
\hline
\end{tabular}

\begin{tabular}{|c|c|c|c|c|}
\hline Compound & $\mathrm{DPDP} \cdot \mathrm{ClO}_{4}$ & PTAP·ClO 4 & TIAP·ClO ${ }_{4}$ & TPAP·IO 4 \\
\hline Chemical formula & $\mathrm{C}_{18} \mathrm{H}_{26} \mathrm{CIN}_{2} \mathrm{O}_{4} \mathrm{P}$ & $\mathrm{C}_{15} \mathrm{H}_{29} \mathrm{ClN}_{3} \mathrm{O}_{4} \mathrm{P}$ & $\mathrm{C}_{12} \mathrm{H}_{32} \mathrm{ClN}_{4} \mathrm{O}_{4} \mathrm{P}$ & $\mathrm{C}_{21} \mathrm{H}_{23} \mathrm{INO}_{3.99} \mathrm{P}$ \\
\hline Formula weight & 400.83 & 381.83 & 362.83 & 510.84 \\
\hline Temperature & $125(2) \mathrm{K}$ & $100(2) \mathrm{K}$ & $100(2) \mathrm{K}$ & $100(2) \mathrm{K}$ \\
\hline Crystal system & Monoclinic & Monoclinic & Tetragonal & Monoclinic \\
\hline Space group & Cc & $P 2_{1}$ & $I-42 m$ & $P 2_{1} / c$ \\
\hline $\mathrm{a}(\AA) ; \alpha\left(^{\circ}\right)$ & $15.5855(18) ; 90$ & $9.368(2) ; 90$ & $9.9290(12) ; 90$ & $12.622(3) ; 90$ \\
\hline$b(\AA) ; \beta\left(^{\circ}\right)$ & $9.0967(11) ; 102.867(3)$ & $15.391(3) ; 94.152(5)$ & $9.9290(12) ; 90$ & $7.5999(17) ; 90.525(5)$ \\
\hline$c(\AA) ; \gamma\left({ }^{\circ}\right)$ & $14.7829(17) ; 90$ & $27.587(6) ; 90$ & $9.7163(12) ; 90$ & $22.535(5) ; 90$ \\
\hline $\mathrm{V}\left(\AA^{3}\right) ; \mathrm{Z}$ & $2043.2(4) ; 4$ & $3966.9(15) ; 8$ & $957.9(3) ; 2$ & $2161.6(8) ; 4$ \\
\hline$\rho$ (calc.) $\mathrm{g} \mathrm{cm}^{-3}$ & 1.303 & 1.279 & 1.258 & 1.570 \\
\hline$\mu\left(\mathrm{Mo} \mathrm{K}_{\alpha}\right) \mathrm{mm}^{-1}$ & 0.290 & 0.296 & 0.304 & 1.578 \\
\hline $2 \theta_{\max }\left({ }^{\circ}\right)$ & 56.54 & 52.74 & 56.58 & 50.06 \\
\hline $\mathrm{R}$ (int) & 0.0568 & 0.1099 & 0.0493 & 0.0643 \\
\hline Completeness to $\theta$ & 100 & 100 & 100 & 99.9 \\
\hline Data / param. & $4506 / 211$ & $16216 / 866$ & $650 / 32$ & $3814 / 288$ \\
\hline GOF & 1.030 & 1.005 & 1.116 & 1.464 \\
\hline $\mathrm{R} 1[\mathrm{~F}>4 \sigma(\mathrm{F})]$ & 0.0409 & 0.0515 & 0.0312 & 0.0708 \\
\hline wR2 (all data) & 0.1012 & 0.1074 & 0.0761 & 0.1663 \\
\hline max. peak/hole $\left(e . \AA^{-3}\right)$ & $0.574 /-0.339$ & $0.294 /-0.335$ & $0.246 /-0.349$ & $1.883 /-2.719$ \\
\hline
\end{tabular}




\begin{tabular}{|l|l|l|l|}
\hline Compound & DPDP·IO ${ }_{4}$ & PTAP·IO & TIAP·IO \\
\hline
\end{tabular}

Table S2. Summary of the space groups of all the tetrahedral anion supported phosphonium salts.

\begin{tabular}{|c|c|c|c|}
\hline S.No & $\begin{array}{c}\text { Ferro and Piezoelectric } \\
\text { Materials }\end{array}$ & $\begin{array}{l}\text { Temperature } \\
\text { (K) }\end{array}$ & Space Group \\
\hline 1 & TPAP·BF 4 & 100 & Monoclinic $P n$ \\
\hline 2 & $\mathrm{DPDP} \cdot \mathrm{BF}_{4}$ & $\begin{array}{l}100 \\
150\end{array}$ & $\begin{array}{l}\text { Monoclinic } P n \\
\text { Monoclinic } C c\end{array}$ \\
\hline 3 & PTAP·BF 4 & 100 & Monoclinic $P 2{ }_{1} / n$ \\
\hline 4 & TIAP·BF 4 & 100 & Tetragonal $I-42 m$ \\
\hline 5 & TPAP. $\mathrm{CIO}_{4}$ & $\begin{array}{l}150 \\
200\end{array}$ & $\begin{array}{l}\text { Monoclinic } P c \\
\text { Monoclinic } P n\end{array}$ \\
\hline 6 & $\mathrm{DPDP} \cdot \mathrm{ClO}_{4}$ & $\begin{array}{l}100 \\
296\end{array}$ & $\begin{array}{l}\text { Monoclinic } P n \\
\text { Monoclinic } C c\end{array}$ \\
\hline 7 & PTAP. $\mathrm{ClO}_{4}$ & 100 & Monoclinic $P 2_{1}$ \\
\hline 8 & TIAP. $\mathrm{ClO}_{4}$ & 100 & Tetragonal $1-42 m$ \\
\hline 9 & TPAP·IO ${ }_{4}$ & 100 & Monoclinic $P 2_{1} / C$ \\
\hline 10 & $\mathrm{DPDP} \cdot 1 \mathrm{O}_{4}$ & 100 & Monoclinic $C c$ \\
\hline 11 & PTAP $\cdot 1 O_{4}$ & 100 & Monoclinic $P 2{ }_{1} / n$ \\
\hline 12 & TIAP·IO 4 & 100 & Monoclinic $P 2_{1} / n$ \\
\hline
\end{tabular}


Table S3. Table of Hydrogen bond data for all the tetrahedral anion supported phosphonium salts.

\begin{tabular}{|c|c|c|c|c|c|c|}
\hline Compound & D-H...A & $d(D-H)$ & $d(H \ldots A)$ & $d(D-A)$ & $<\mathrm{DHA}$ & $\begin{array}{l}\text { Symmetry } \\
\text { operations }\end{array}$ \\
\hline $\mathrm{TPAP} \cdot \mathrm{BF}_{4}$ & $N(1)-H(1) \ldots F(1)$ & $0.8599(40)$ & $2.4188(70)$ & $2.9823(84)$ & $123.628(301)$ & $x, y, z$ \\
\hline $\mathrm{DPDP} \cdot \mathrm{BF}_{4}$ & $\mathrm{~N}(2)-\mathrm{H}(2) \ldots \mathrm{F}(1)$ & $0.88(1)$ & $2.2140(56)$ & $2.9955(83)$ & $147.818(398)$ & $x,-1+y, 1+z$ \\
\hline \multirow[t]{2}{*}{$100 \mathrm{~K}$} & $N(3)-H(3) \ldots F(8)$ & $0.88(1)$ & $2.0913(73)$ & $2.9114(89)$ & $154.802(364)$ & $\mathrm{x}, \mathrm{y}, \mathrm{z}$ \\
\hline & $N(1)-H(1) \ldots F(6)$ & $0.88(1)$ & $2.2718(38)$ & $2.9337(68)$ & $131.846(378)$ & $x, y, z$ \\
\hline \multirow{2}{*}{$150 \mathrm{~K}$} & $N(1)-H(1) \ldots F(2)$ & $0.8801(32)$ & $2.2221(71)$ & $3.0405(79)$ & $154.568(260)$ & $0.5+x,-0.5+x, z$ \\
\hline & $N(2)-H(2) \ldots F(1)$ & $0.8793(34)$ & $2.2963(36)$ & $2.9562(50)$ & $131.819(245)$ & $x, y, z$ \\
\hline \multirow{3}{*}{$\mathrm{PTAP} \cdot \mathrm{BF}_{4}$} & $N(1)-H(1) \ldots F(1)$ & $0.8798(11)$ & $2.0983(12)$ & $2.9154(16)$ & $154.13(8)$ & $0.5+x, 1.5-y,-0.5+z$ \\
\hline & $\mathrm{N}(2)-\mathrm{H}(2) \ldots \mathrm{F}(2)$ & $0.88(12)$ & $2.0486(9)$ & $2.8651(15)$ & $153.893(82)$ & $x, y, z$ \\
\hline & $N(3)-H(3) \ldots F(3)$ & $0.8801(12)$ & $2.9427(12)$ & $3.3738(17)$ & $112.099(80)$ & $1.5+x, 1.5-y,-0.5+z$ \\
\hline
\end{tabular}

\begin{tabular}{|c|c|c|c|c|c|c|}
\hline Compound & D-H...A & $d(D-H)$ & $d(H \ldots A)$ & $d(D-A)$ & $<$ DHA & $\begin{array}{l}\text { Symmetry } \\
\text { operations }\end{array}$ \\
\hline \multirow{3}{*}{$\begin{array}{c}\text { TPAP.CIO } \\
150 \mathrm{~K}\end{array}$} & $\mathrm{~N}(1)-\mathrm{H}(1) \ldots \mathrm{O}(1)$ & $0.8808(55)$ & $2.1682(47)$ & $2.9691(69)$ & $150.949(332)$ & $x,-1+y, z$ \\
\hline & $\mathrm{N}(1)-\mathrm{H}(1) \ldots \mathrm{O}(2)$ & $0.8808(55)$ & $2.9266(47)$ & $3.4408(69)$ & $118.986(341)$ & $x,-1+y, z$ \\
\hline & $\mathrm{N}(2)-\mathrm{H}(2) \ldots \mathrm{O}(5)$ & $0.8800(55)$ & $2.2344(46)$ & $3.0125(69)$ & $147.288(329)$ & $x,-1+y, z$ \\
\hline \multirow{2}{*}{$200 K$} & $\mathrm{~N}(1)-\mathrm{H}(1) \ldots \mathrm{O}(1)$ & $0.8798(43)$ & $2.2566(56)$ & $3.2380(89)$ & $120.380(332)$ & $1+x,-1+y, z$ \\
\hline & $\mathrm{N}(1)-\mathrm{H}(1) \ldots \mathrm{O}(2)$ & $0.8798(43)$ & $2.7029(71)$ & $3.0627(74)$ & $152.247(308)$ & $x, y, z$ \\
\hline \multirow{4}{*}{$\begin{array}{c}\text { DPDP. } \mathrm{ClO}_{4} \\
100 \mathrm{~K}\end{array}$} & $\mathrm{~N}(2)-\mathrm{H}(2) \ldots \mathrm{O}(1)$ & $0.8797(34)$ & $2.3243(27)$ & $2.9789(44)$ & $131.274(236)$ & $x, y, z$ \\
\hline & $\mathrm{N}(2)-\mathrm{H}(2) \ldots \mathrm{O}(2)$ & $0.8797(34)$ & $2.7748(45)$ & $3.6035(56)$ & $157.550(227)$ & $x, y, z$ \\
\hline & $\mathrm{N}(3)-\mathrm{H}(3) \ldots \mathrm{O}(4)$ & $0.8792(30)$ & $2.1136(53)$ & $2.9470(62)$ & $157.952(233)$ & $x, y, z$ \\
\hline & $\mathrm{N}(4)-\mathrm{H}(4) \ldots \mathrm{O}(6)$ & $0.8799(31)$ & $2.2788(27)$ & $2.9795(39)$ & $136.522(194)$ & $x,-1+y, z$ \\
\hline $125 \mathrm{~K}$ & $\mathrm{~N}(1)-\mathrm{H}(1) \ldots \mathrm{O}(1)$ & $0.8596(61)$ & $2.9732(195)$ & $3.8078(206)$ & $164.288(557)$ & $-0.5+x, 0.5+y, z$ \\
\hline \multirow{10}{*}{ PTAP. $\mathrm{CIO}_{4}$} & $\mathrm{~N}(3)-\mathrm{H}(3) \ldots \mathrm{O}(12)$ & $0.88000(4)$ & $2.3334(38)$ & $3.0057(55)$ & $133.266(266)$ & $-1+x, y, z$ \\
\hline & $\mathrm{N}(4)-\mathrm{H}(4) \ldots \mathrm{O}(5)$ & $0.8795(44)$ & $2.1698(42)$ & $2.9672(61)$ & $150.514(304)$ & $1+x, y, z$ \\
\hline & $\mathrm{N}(5)-\mathrm{H}(5) \ldots \mathrm{O}(16)$ & $0.8789(46)$ & $2.2204(32)$ & $3.0327(56)$ & $153.563(306)$ & $x,-1+y, z$ \\
\hline & $\mathrm{N}(6)-\mathrm{H}(6) \ldots \mathrm{O}(7)$ & $0.8793(44)$ & $2.2627(37)$ & $3.0241(54)$ & $144.815(279)$ & $1+x, y, z$ \\
\hline & $\mathrm{N}(7)-\mathrm{H}(7) \ldots \mathrm{O}(8)$ & $0.8807(43)$ & $2.1906(32)$ & $3.0403(54)$ & 161.990(294) & $x, y, z$ \\
\hline & $\mathrm{N}(8)-\mathrm{H}(8) \ldots \mathrm{O}(15)$ & $0.8812(40)$ & $2.1502(40)$ & $2.9992(57)$ & $161.573(284)$ & $-1+x, y, z$ \\
\hline & $\mathrm{N}(9)-\mathrm{H}(9) \ldots \mathrm{O}(5)$ & $0.8805(41)$ & $2.4120(43)$ & $2.9901(61)$ & $123.551(293)$ & $x, y, z$ \\
\hline & $\mathrm{N}(10)-\mathrm{H}(10) \ldots \mathrm{O}(2)$ & $0.8801(46)$ & $2.3208(45)$ & $3.1841(64)$ & $166.835(320)$ & $1-x, 0.5+y, 1-z$ \\
\hline & $\mathrm{N}(10)-\mathrm{H}(10) \ldots \mathrm{O}(4)$ & $0.8801(46)$ & $2.6195(41)$ & $3.2559(63)$ & $130.015(315)$ & $1-x, 0.5+y, 1-z$ \\
\hline & $\mathrm{N}(11)-\mathrm{H}(11) \ldots \mathrm{O}(3)$ & $0.8808(46)$ & $2.1776(36)$ & $2.9610(58)$ & $147.903(316)$ & $x, y,-1+z$ \\
\hline
\end{tabular}




\begin{tabular}{|c|c|c|c|c|c|c|}
\hline Compound & D-H...A & $d(D-H)$ & $d(H \ldots A)$ & $d(D-A)$ & $<$ DHA & Symmetry operations \\
\hline $\mathrm{DPDP} \cdot 1 \mathrm{O}_{4}$ & $\begin{array}{l}\mathrm{N}(1)-\mathrm{H}(1) \ldots \mathrm{O}(1) \\
\mathrm{N}(2)-\mathrm{H}(2) \ldots \mathrm{O}(4)\end{array}$ & $\begin{array}{l}0.8809(29) \\
0.8797(29)\end{array}$ & $\begin{array}{l}2.2193(20) \\
2.1866(31)\end{array}$ & $\begin{array}{l}2.8954(34) \\
2.9912(43)\end{array}$ & $\begin{array}{l}133.307(188) \\
151.852(200)\end{array}$ & $\begin{array}{c}x, y, z \\
0.5+x, 0.5+y, z\end{array}$ \\
\hline PTAP $\cdot 1 O_{4}$ & $\begin{array}{l}\mathrm{N}(1)-\mathrm{H}(1) \ldots \mathrm{O}(4) \\
\mathrm{N}(2)-\mathrm{H}(2) \ldots \mathrm{O}(3) \\
\mathrm{N}(3)-\mathrm{H}(3) \ldots \mathrm{O}(2)\end{array}$ & $\begin{array}{l}0.8798(17) \\
0.8795(16) \\
0.8801(17)\end{array}$ & $\begin{array}{l}2.3333(16) \\
2.3564(17) \\
2.1978(17)\end{array}$ & $\begin{array}{l}3.1063(24) \\
3.1858(24) \\
2.9224(24)\end{array}$ & $\begin{array}{l}146.688(188) \\
157.279(115) \\
139.352(120)\end{array}$ & $\begin{array}{c}0.5-x, 0.5+y, 1.5-z \\
-0.5+x, 0.5-y, 0.5+z \\
0.5-x, 0.5+y, 1.5-z\end{array}$ \\
\hline TIAP $\cdot I_{4}$ & $\begin{array}{l}\mathrm{N}(1)-\mathrm{H}(1) \ldots \mathrm{O}(2) \\
\mathrm{N}(2)-\mathrm{H}(2) \ldots \mathrm{O}(3) \\
\mathrm{N}(3)-\mathrm{H}(3) \ldots \mathrm{O}(1) \\
\mathrm{N}(4)-\mathrm{H}(4) \ldots \mathrm{O}(4)\end{array}$ & $\begin{array}{l}0.8796(14) \\
0.8796(15) \\
0.8804(14) \\
0.8797(13)\end{array}$ & $\begin{array}{l}2.1094(14) \\
2.3735(12) \\
2.2206(13) \\
2.4124(15)\end{array}$ & $\begin{array}{l}2.9381(20) \\
2.9391(19) \\
3.0689(19) \\
3.0001(20)\end{array}$ & $\begin{array}{l}156.747(97) \\
122.299(92) \\
161.663(99) \\
124.557(93)\end{array}$ & $\begin{array}{c}-0.5+x, 1.5-y,-0.5+z \\
-1+x, y, z \\
x, y, z \\
1.5-x, 0.5+y, 1.5-z\end{array}$ \\
\hline
\end{tabular}

Table S4. Selected bond lengths $[\AA]$ and angles $\left[{ }^{\circ}\right]$ for all the tetrahedral anion supported phosphonium salts.

\begin{tabular}{|c|c|c|}
\hline Compound & Bond length & Bond angle \\
\hline $\mathrm{TPAP} \cdot \mathrm{BF}_{4}$ & $\begin{array}{l}\mathrm{P}(1)-\mathrm{N}(1): 1.6160(37) \\
\mathrm{N}(1)-\mathrm{H}(1): 0.8599(40)\end{array}$ & $\begin{array}{ll}\mathrm{N}(1)-\mathrm{P}(1)-\mathrm{C}(11) & : 106.617(181) \\
\mathrm{N}(1)-\mathrm{P}(1)-\mathrm{C}(21) & : 114.167(181) \\
\mathrm{N}(1)-\mathrm{P}(1)-\mathrm{C}(31) & : 109.966(167)\end{array}$ \\
\hline $\begin{array}{l}\text { DPDP. BF } \\
100 \mathrm{~K}\end{array}$ & $\begin{array}{l}\mathrm{P}(1)-\mathrm{N}(1): 1.6192(49) \\
\mathrm{P}(1)-\mathrm{N}(2): 1.6286(66) \\
\mathrm{P}(2)-\mathrm{N}(3): 1.6183(53) \\
\mathrm{P}(2)-\mathrm{N}(4): 1.6313(43) \\
\mathrm{N}(1)-\mathrm{H}(1): 0.8808(61) \\
\mathrm{N}(2)-\mathrm{H}(2): 0.8798(60) \\
\mathrm{N}(3)-\mathrm{H}(3): 0.8796(52) \\
\mathrm{N}(4)-\mathrm{H}(4): 0.8799(48)\end{array}$ & $\begin{array}{ll}\mathrm{N}(1)-\mathrm{P}(1)-\mathrm{C}(21) & : 105.634(269) \\
\mathrm{N}(1)-\mathrm{P}(1)-\mathrm{C}(31) & : 108.055(282) \\
\mathrm{N}(2)-\mathrm{P}(1)-\mathrm{C}(21) & : 107.435(299) \\
\mathrm{N}(2)-\mathrm{P}(1)-\mathrm{C}(31) & : 104.395(282) \\
\mathrm{N}(3)-\mathrm{P}(2)-\mathrm{C}(41) & : 103.835(280) \\
\mathrm{N}(3)-\mathrm{P}(2)-\mathrm{C}(51) & : 107.636(268) \\
\mathrm{N}(4)-\mathrm{P}(2)-\mathrm{C}(41) & : 109.984(264) \\
\mathrm{N}(4)-\mathrm{P}(2)-\mathrm{C}(51) & : 104.811(263)\end{array}$ \\
\hline $150 \mathrm{~K}$ & $\begin{array}{l}\mathrm{P}(1)-\mathrm{N}(1): 1.6223(35) \\
\mathrm{P}(1)-\mathrm{N}(2): 1.6225(28) \\
\mathrm{N}(1)-\mathrm{H}(1): 0.8801(32) \\
\mathrm{N}(2)-\mathrm{H}(2): 0.8793(34)\end{array}$ & $\begin{array}{ll}\mathrm{N}(1)-\mathrm{P}(1)-\mathrm{C}(11) & : 104.268(129) \\
\mathrm{N}(1)-\mathrm{P}(1)-\mathrm{C}(21) & : 107.276(154) \\
\mathrm{N}(2)-\mathrm{P}(1)-\mathrm{C}(11) & : 109.089(149) \\
\mathrm{N}(2)-\mathrm{P}(1)-\mathrm{C}(21) & : 105.455(130)\end{array}$ \\
\hline PTAP $\cdot \mathrm{BF}_{4}$ & $\begin{array}{l}\mathrm{P}(1)-\mathrm{N}(1): 1.6186(12) \\
\mathrm{P}(1)-\mathrm{N}(2): 1.6207(13) \\
\mathrm{P}(1)-\mathrm{N}(3): 1.6227(12) \\
\mathrm{N}(1)-\mathrm{H}(1): 0.8798(11) \\
\mathrm{N}(2)-\mathrm{H}(2): 0.8800(12) \\
\mathrm{N}(3)-\mathrm{H}(3): 0.8801(12)\end{array}$ & $\begin{array}{ll}\mathrm{N}(1)-\mathrm{P}(1)-\mathrm{C}(11) & : 107.251(51) \\
\mathrm{N}(2)-\mathrm{P}(1)-\mathrm{C}(11) & : 103.842(55) \\
\mathrm{N}(3)-\mathrm{P}(1)-\mathrm{C}(11) & : 115.903(52)\end{array}$ \\
\hline $\mathrm{TIAP} \cdot \mathrm{BF}_{4}$ & $\begin{array}{l}P(1)-N(1): 1.6099(14) \\
N(1)-H(1): 0.8798(16)\end{array}$ & $\mathrm{N}(1)-\mathrm{P}(1)-\mathrm{C}(1) \quad:$ 127.410(96) \\
\hline
\end{tabular}




\begin{tabular}{|c|c|c|}
\hline Compound & Bond length & Bond angle \\
\hline $\begin{array}{l}\text { TPAP. } \mathrm{CIO}_{4} \\
150 \mathrm{~K}\end{array}$ & $\begin{array}{l}P(1)-N(1): 1.6200(47) \\
P(1)-N(2): 1.6293(47) \\
N(1)-H(1): 0.8808(55) \\
N(1)-H(1): 0.8800(55)\end{array}$ & $\begin{array}{ll}\mathrm{N}(1)-\mathrm{P}(1)-\mathrm{C}(11) & : 109.457(275) \\
\mathrm{N}(1)-\mathrm{P}(1)-\mathrm{C}(21) & : 113.496(272) \\
\mathrm{N}(1)-\mathrm{P}(1)-\mathrm{C}(31) & : 106.917(274) \\
\mathrm{N}(2)-\mathrm{P}(2)-\mathrm{C}(41) & : 115.301(271) \\
\mathrm{N}(2)-\mathrm{P}(2)-\mathrm{C}(51) & : 106.871(280) \\
\mathrm{N}(2)-\mathrm{P}(2)-\mathrm{C}(61) & : 109.129(276)\end{array}$ \\
\hline $200 K$ & $\begin{array}{l}P(1)-N(1): 1.6093(46) \\
N(1)-H(1): 0.8798(43)\end{array}$ & $\begin{array}{ll}N(1)-P(1)-C(11) & : 114.406(237) \\
N(1)-P(1)-C(21) & : 109.399(248) \\
N(1)-P(1)-C(31) & : 106.613(230)\end{array}$ \\
\hline $\begin{array}{l}\text { DPDP.ClO } \\
100 \mathrm{~K}\end{array}$ & $\begin{array}{l}\mathrm{P}(1)-\mathrm{N}(1): 1.6278(34) \\
\mathrm{P}(1)-\mathrm{N}(2): 1.6156(28) \\
\mathrm{P}(2)-\mathrm{N}(3): 1.6195(34) \\
\mathrm{P}(2)-\mathrm{N}(4): 1.6229(28) \\
\mathrm{N}(1)-\mathrm{H}(1): 0.8798(35) \\
\mathrm{N}(2)-\mathrm{H}(2): 0.8797(34) \\
\mathrm{N}(3)-\mathrm{H}(3): 0.8792(30) \\
\mathrm{N}(4)-\mathrm{H}(4): 0.8799(31)\end{array}$ & $\begin{array}{ll}\mathrm{N}(1)-\mathrm{P}(1)-\mathrm{C}(21) & : 104.64(17) \\
\mathrm{N}(1)-\mathrm{P}(1)-\mathrm{C}(31) & : 107.190(154) \\
\mathrm{N}(2)-\mathrm{P}(1)-\mathrm{C}(21) & : 107.793(174) \\
\mathrm{N}(2)-\mathrm{P}(1)-\mathrm{C}(31) & : 105.864(155) \\
\mathrm{N}(3)-\mathrm{P}(1)-\mathrm{C}(41) & : 104.382(148) \\
\mathrm{N}(3)-\mathrm{P}(1)-\mathrm{C}(51) & : 107.634(152) \\
\mathrm{N}(4)-\mathrm{P}(1)-\mathrm{C}(41) & : 109.134(148) \\
\mathrm{N}(4)-\mathrm{P}(1)-\mathrm{C}(51) & : 104.91(15)\end{array}$ \\
\hline $125 \mathrm{~K}$ & $\begin{array}{l}P(1)-N(1): 1.6211(50) \\
P(1)-N(2): 1.6138(52) \\
N(1)-H(1): 0.8596(61) \\
N(2)-H(2): 0.8603(49)\end{array}$ & $\begin{array}{ll}N(1)-P(1)-C(11) & : 109.268(276) \\
N(1)-P(1)-C(21) & : 105.993(229) \\
N(2)-P(1)-C(11) & : 104.574(266) \\
N(2)-P(1)-C(21) & : 106.735(276)\end{array}$ \\
\hline PTAP. $\mathrm{ClO}_{4}$ & $\begin{array}{l}\mathrm{P}(1)-\mathrm{N}(1): 1.6218(40) \\
\mathrm{P}(1)-\mathrm{N}(2): 1.6198(42) \\
\mathrm{P}(1)-\mathrm{N}(3): 1.6117(40) \\
\mathrm{N}(1)-\mathrm{H}(1): 0.8804(40) \\
\mathrm{N}(2)-\mathrm{H}(2): 0.8796(39) \\
\mathrm{N}(3)-\mathrm{H}(3): 0.88000(4) \\
\mathrm{P}(2)-\mathrm{N}(4): 1.6161(46) \\
\mathrm{P}(2)-\mathrm{N}(5): 1.6066(40) \\
\mathrm{P}(2)-\mathrm{N}(6): 1.6260(41) \\
\mathrm{N}(4)-\mathrm{H}(4): 0.8795(44) \\
\mathrm{N}(5)-\mathrm{H}(5): 0.8789(46) \\
\mathrm{N}(6)-\mathrm{H}(6): 0.8793(44) \\
\mathrm{P}(3)-\mathrm{N}(7): 1.6137(39) \\
\mathrm{P}(3)-\mathrm{N}(8): 1.6086(47) \\
\mathrm{P}(3)-\mathrm{N}(9): 1.6316(46) \\
\mathrm{N}(7)-\mathrm{H}(7): 0.8807(43) \\
\mathrm{N}(8)-\mathrm{H}(8): 0.8812(40) \\
\mathrm{N}(9)-\mathrm{H}(9): 0.8805(41) \\
\mathrm{P}(4)-\mathrm{N}(10): 1.6195(48) \\
\mathrm{P}(4)-\mathrm{N}(11): 1.6284(41) \\
\mathrm{P}(4)-\mathrm{N}(12): 1.6166(45) \\
\mathrm{N}(10)-\mathrm{H}(10): 0.8798(35) \\
\mathrm{N}(11)-\mathrm{H}(11): 0.8797(34) \\
\mathrm{N}(12)-\mathrm{H}(12): 0.8792(30)\end{array}$ & $\begin{array}{rc}\mathrm{N}(1)-\mathrm{P}(1)-\mathrm{C}(1 \mathrm{P}) & : 108.982(233) \\
\mathrm{N}(2)-\mathrm{P}(1)-\mathrm{C}(1 \mathrm{P}) & : 113.675(235) \\
\mathrm{N}(3)-\mathrm{P}(1)-\mathrm{C}(1 \mathrm{P}) & : 105.236(238) \\
\mathrm{N}(4)-\mathrm{P}(2)-\mathrm{C}(7 \mathrm{P}) & : 110.771(230) \\
\mathrm{N}(5)-\mathrm{P}(2)-\mathrm{C}(7 \mathrm{P}) & : 104.575(221) \\
\mathrm{N}(6)-\mathrm{P}(2)-\mathrm{C}(7 \mathrm{P}) & : 113.128(225) \\
\mathrm{N}(7)-\mathrm{P}(3)-\mathrm{C}(13 \mathrm{P}) & : 110.053(219) \\
\mathrm{N}(8)-\mathrm{P}(3)-\mathrm{C}(13 \mathrm{P}) & : 106.995(218) \\
\mathrm{N}(9)-\mathrm{P}(3)-\mathrm{C}(13 \mathrm{P}) & : 113.398(219) \\
\mathrm{N}(10)-\mathrm{P}(4)-\mathrm{C}(18 \mathrm{P}) & : 103.791(262) \\
\mathrm{N}(11)-\mathrm{P}(4)-\mathrm{C}(18 \mathrm{P}) & : 109.808(242) \\
\mathrm{N}(12)-\mathrm{P}(4)-\mathrm{C}(18 \mathrm{P}) & : 114.641(240)\end{array}$ \\
\hline $\mathrm{TIAP} \cdot \mathrm{CIO}_{4}$ & $\begin{array}{l}P(1)-N(1): 1.5954(78) \\
N(1)-H(1): 0.8594(86)\end{array}$ & $N(1)-P(1)-C(1) \quad: 129.525(612)$ \\
\hline
\end{tabular}




\begin{tabular}{|c|c|c|}
\hline Compound & Bond length & Bond angle \\
\hline TPAP $\cdot 1 O_{4}$ & $\begin{array}{c}\mathrm{P}(1)-\mathrm{N}(1): 1.6204(72) \\
\mathrm{N}(1)-\mathrm{H}(1): 0.879(6)\end{array}$ & $\begin{array}{ll}N(1)-P(1)-C(11) & : 118.039(308) \\
N(1)-P(1)-C(21) & : 108.992(289) \\
N(1)-P(1)-C(31) & : 111.720(279)\end{array}$ \\
\hline DPDP.1O ${ }_{4}$ & $\begin{array}{l}\mathrm{P}(1)-\mathrm{N}(1): 1.6196(25) \\
\mathrm{P}(1)-\mathrm{N}(2): 1.6149(32) \\
\mathrm{N}(1)-\mathrm{H}(1): 0.8809(29) \\
\mathrm{N}(1)-\mathrm{H}(2): 0.8797(29)\end{array}$ & $\begin{array}{ll}N(1)-P(1)-C(11) & : 105.448(121) \\
N(1)-P(1)-C(21) & : 106.732(126) \\
N(2)-P(1)-C(11) & : 108.857(120) \\
N(2)-P(1)-C(21) & : 105.907(107)\end{array}$ \\
\hline PTAP $\cdot 1 O_{4}$ & $\begin{array}{l}\mathrm{P}(1)-\mathrm{N}(1): 1.6250(17) \\
\mathrm{P}(1)-\mathrm{N}(2): 1.6225(18) \\
\mathrm{P}(1)-\mathrm{N}(3): 1.6232(18) \\
\mathrm{N}(1)-\mathrm{H}(1): 0.8798(17) \\
\mathrm{N}(2)-\mathrm{H}(2): 0.8795(16) \\
\mathrm{N}(3)-\mathrm{H}(3): 0.8801(17)\end{array}$ & $\begin{array}{ll}N(1)-P(1)-C(11) & : 104.169(89) \\
N(2)-P(1)-C(11) & : 105.622(93) \\
N(3)-P(1)-C(11) & : 116.134(92)\end{array}$ \\
\hline TIAP $\cdot I_{4}$ & $\begin{array}{l}\mathrm{P}(1)-\mathrm{N}(1): 1.6214(14) \\
\mathrm{P}(1)-\mathrm{N}(2): 1.6247(14) \\
\mathrm{P}(1)-\mathrm{N}(3): 1.6227(14) \\
\mathrm{P}(1)-\mathrm{N}(4): 1.6269(14) \\
\mathrm{N}(1)-\mathrm{H}(1): 0.8796(14) \\
\mathrm{N}(2)-\mathrm{H}(2): 0.8796(15) \\
\mathrm{N}(3)-\mathrm{H}(3): 0.8804(14) \\
\mathrm{N}(4)-\mathrm{H}(4): 0.8797(13)\end{array}$ & $\begin{array}{cc}\mathrm{N}(1)-\mathrm{P}(1)-\mathrm{C}(1) & : 125.364(114) \\
\mathrm{N}(2)-\mathrm{P}(1)-\mathrm{C}(4) & : 126.479(107) \\
\mathrm{N}(3)-\mathrm{P}(1)-\mathrm{C}(7) & : 124.498(114) \\
\mathrm{N}(4)-\mathrm{P}(1)-\mathrm{C}(10) & : 128.376(110)\end{array}$ \\
\hline
\end{tabular}

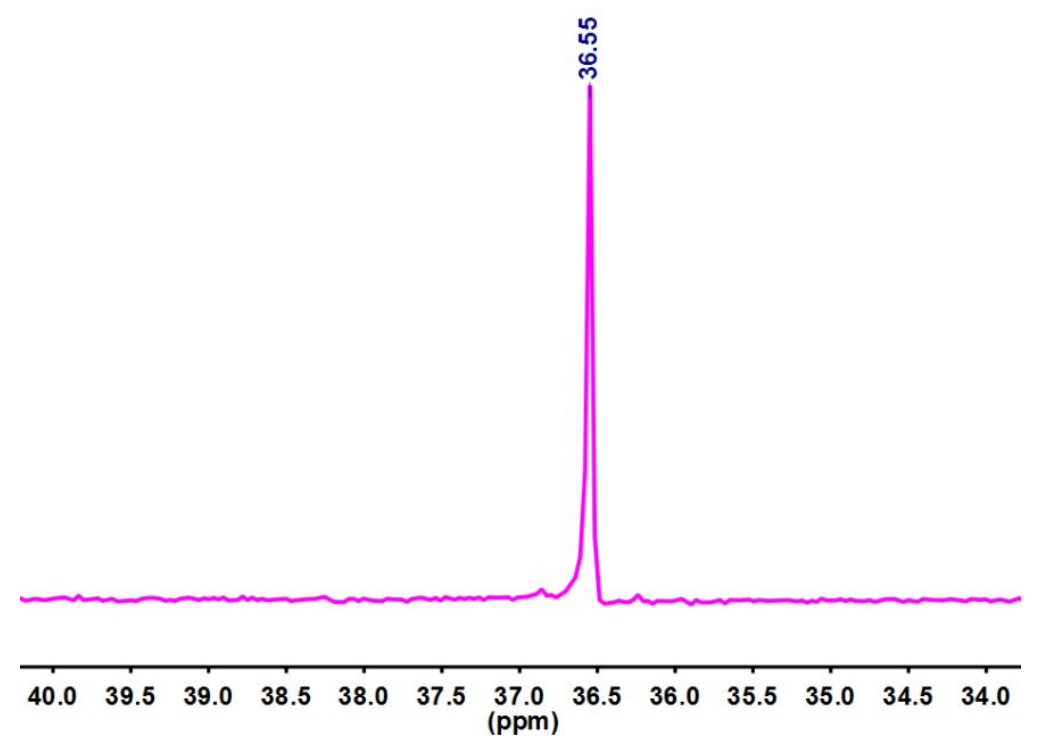

Figure S1. ${ }^{31} \mathrm{P}$ NMR spectrum of TPAP $\cdot \mathrm{BF}_{4}$ 


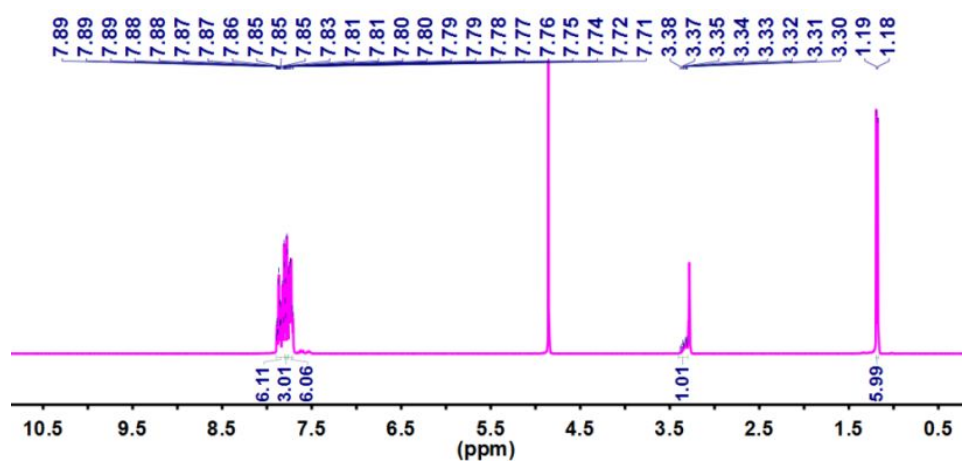

Figure S2. ${ }^{1} \mathrm{H}$ NMR spectrum of TPAP·BF ${ }_{4}$

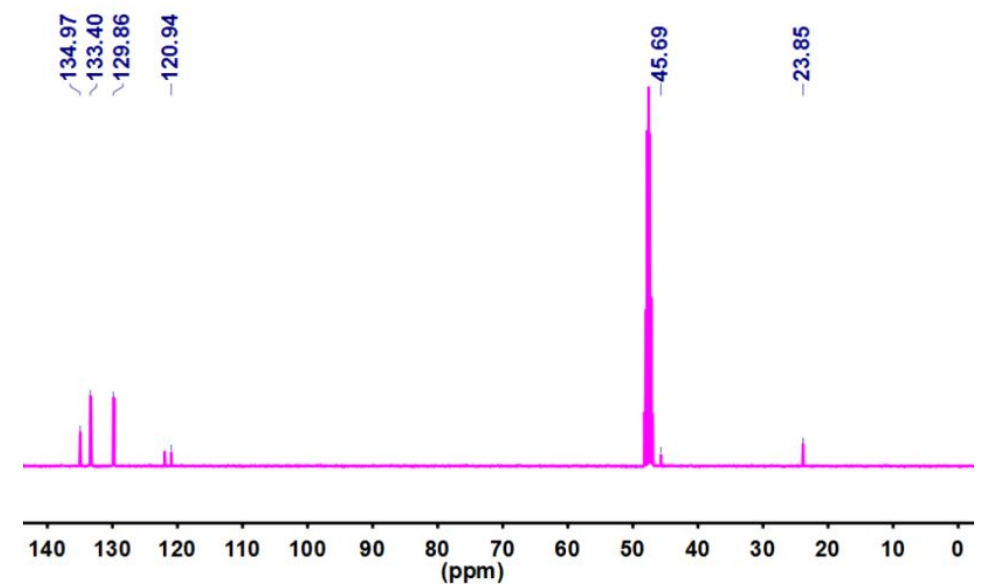

Figure S3. ${ }^{13} \mathrm{C}$ NMR spectrum of TPAP. $\mathrm{BF}_{4}$

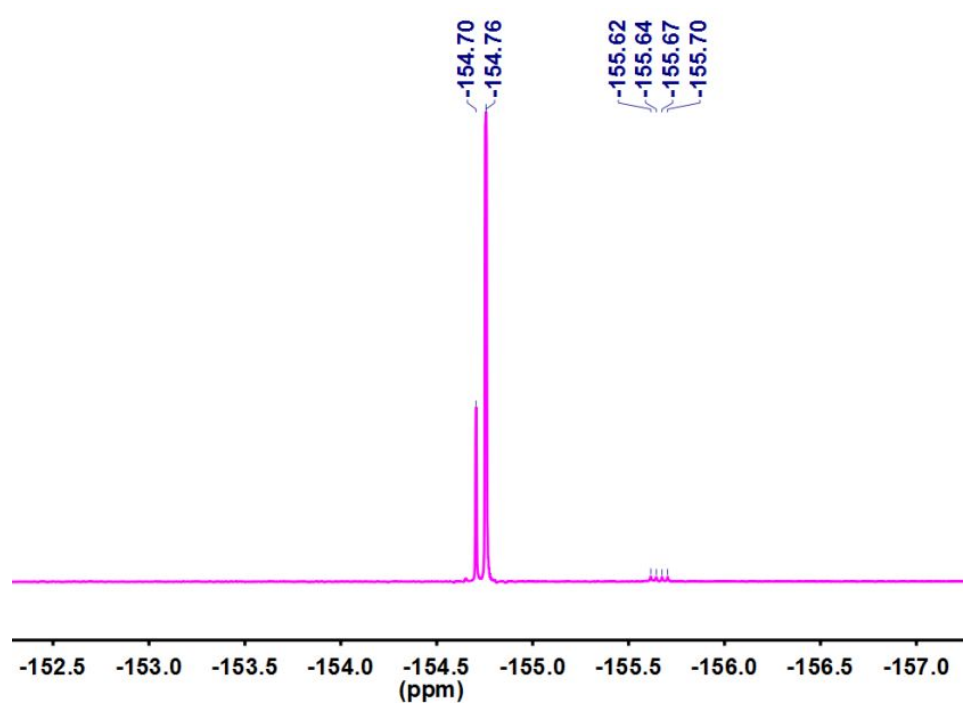

Figure S4. ${ }^{19} \mathrm{~F}$ NMR spectrum of TPAP.BF 


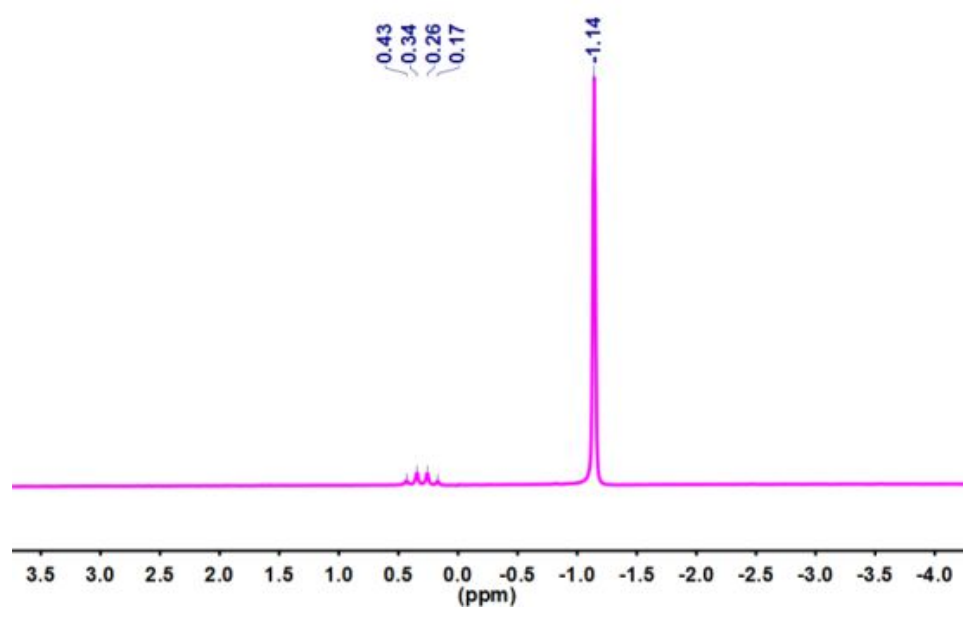

Figure S5. ${ }^{11} \mathrm{~B}$ NMR spectrum of TPAP $\cdot \mathrm{BF}_{4}$

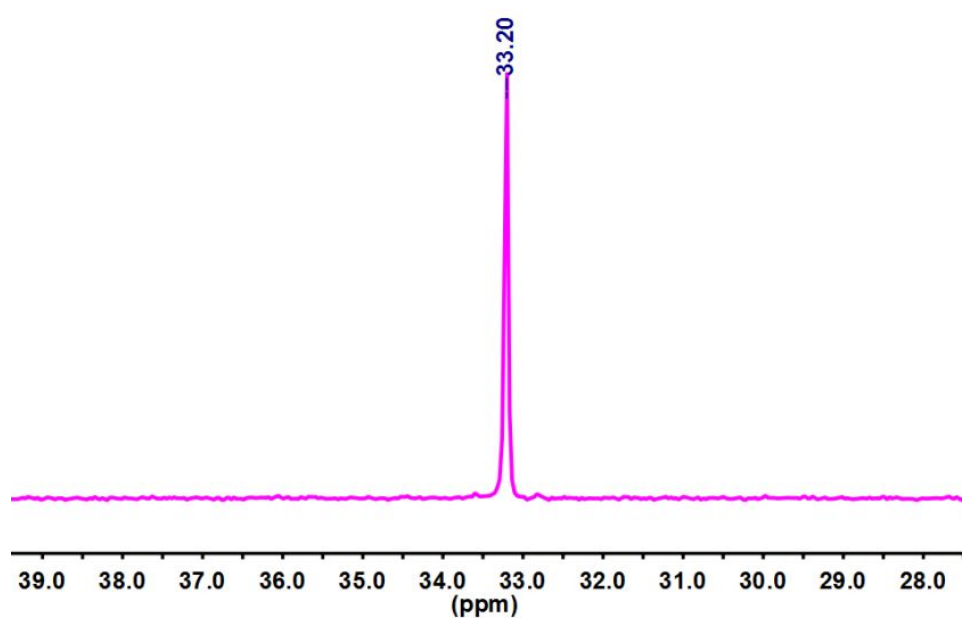

Figure S6. ${ }^{31} \mathrm{P}$ NMR spectrum of DPDP. $\mathrm{BF}_{4}$

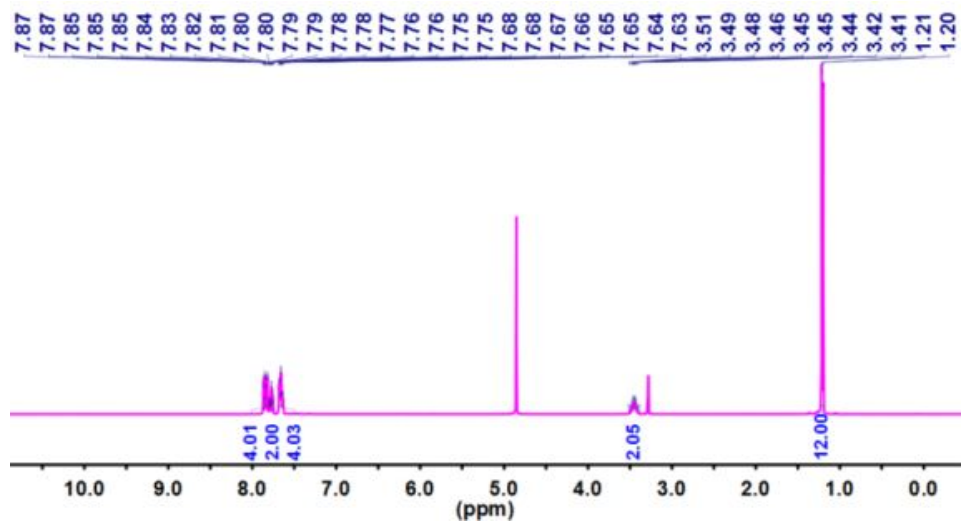

Figure S7. ${ }^{1} \mathrm{H}$ NMR spectrum of DPDP. $\mathrm{BF}_{4}$ 


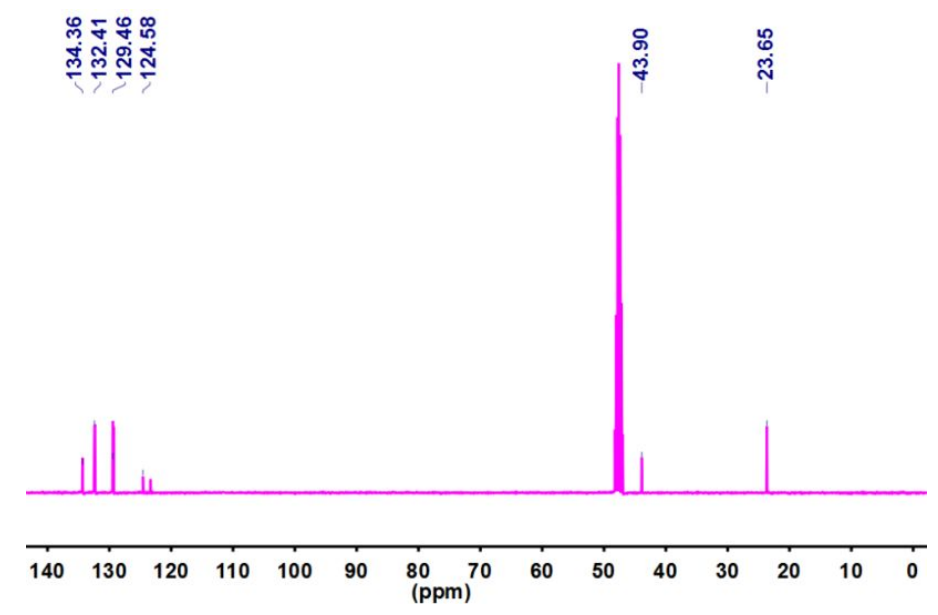

Figure S8. ${ }^{13} \mathrm{C}$ NMR spectrum of DPDP. $\mathrm{BF}_{4}$

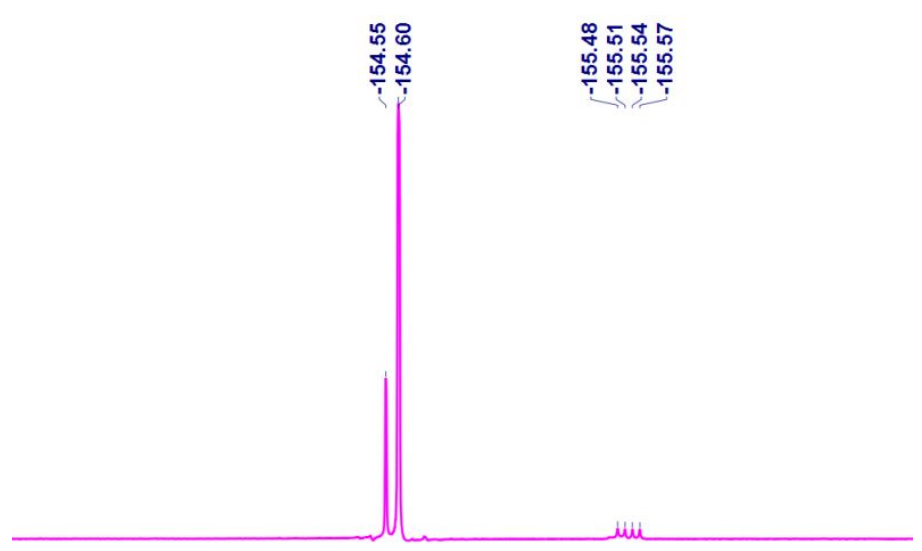

\begin{tabular}{llllllllllll}
\hline-153.2 & -153.5 & -153.8 & -154.1 & -154.4 & -154.7 & -155.0 & -155.3 & -155.6 & -155.9 & -156.2 & -156.5
\end{tabular} (ppm)

Figure S9. ${ }^{19} \mathrm{~F}$ NMR spectrum of DPDP.BF 4

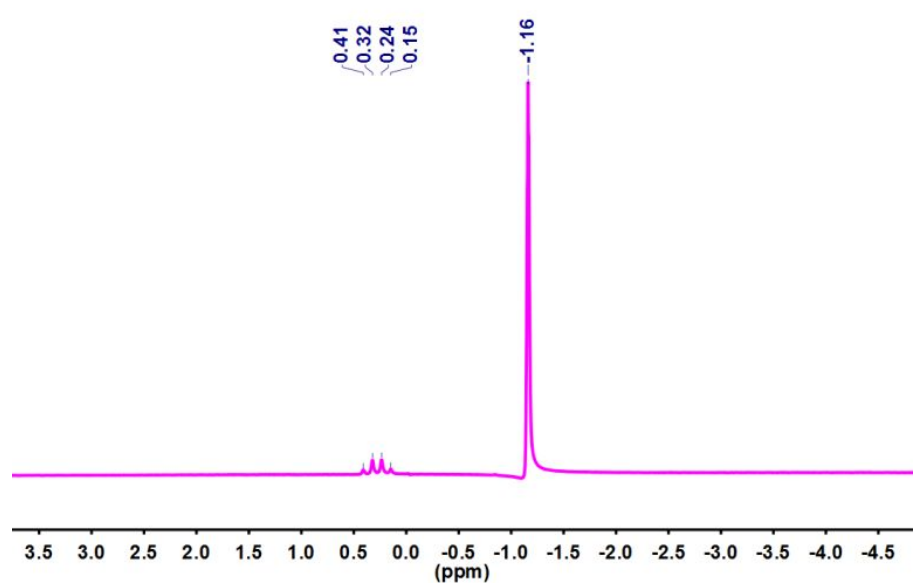

Figure S10. ${ }^{11} \mathrm{~B}$ NMR spectrum of DPDP.BF 4 


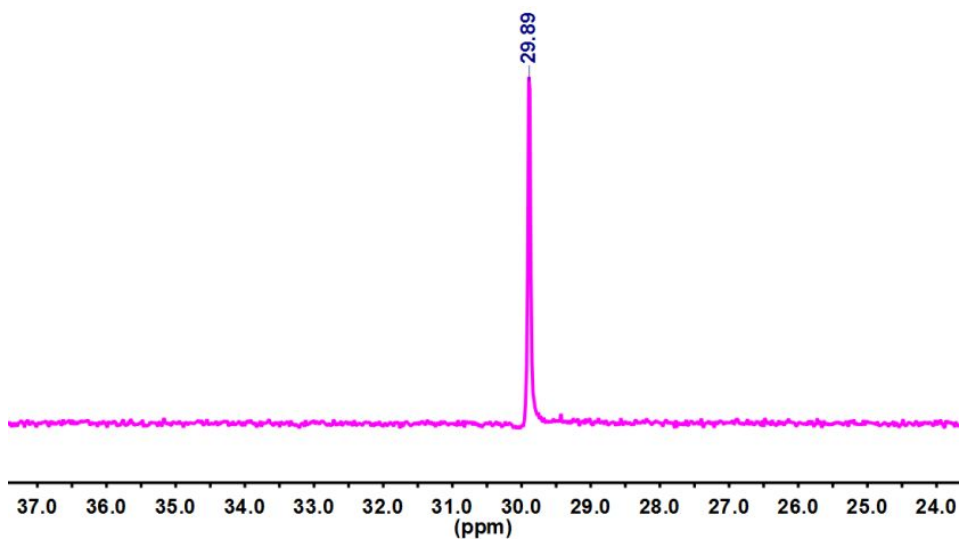

Figure S11. ${ }^{31} \mathrm{P}$ NMR spectrum of PTAP.BF 4

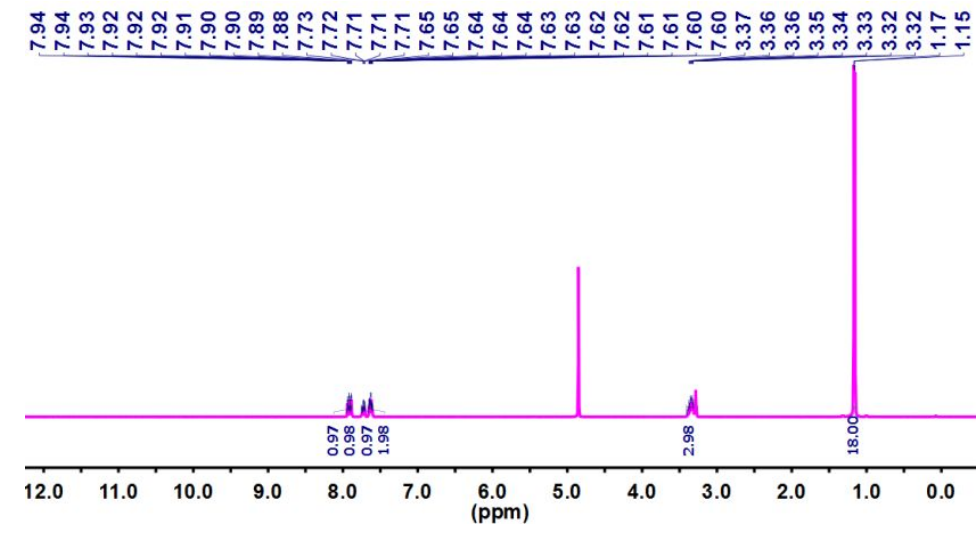

Figure S12. ${ }^{1} \mathrm{H}$ NMR spectrum of PTAP.BF 4

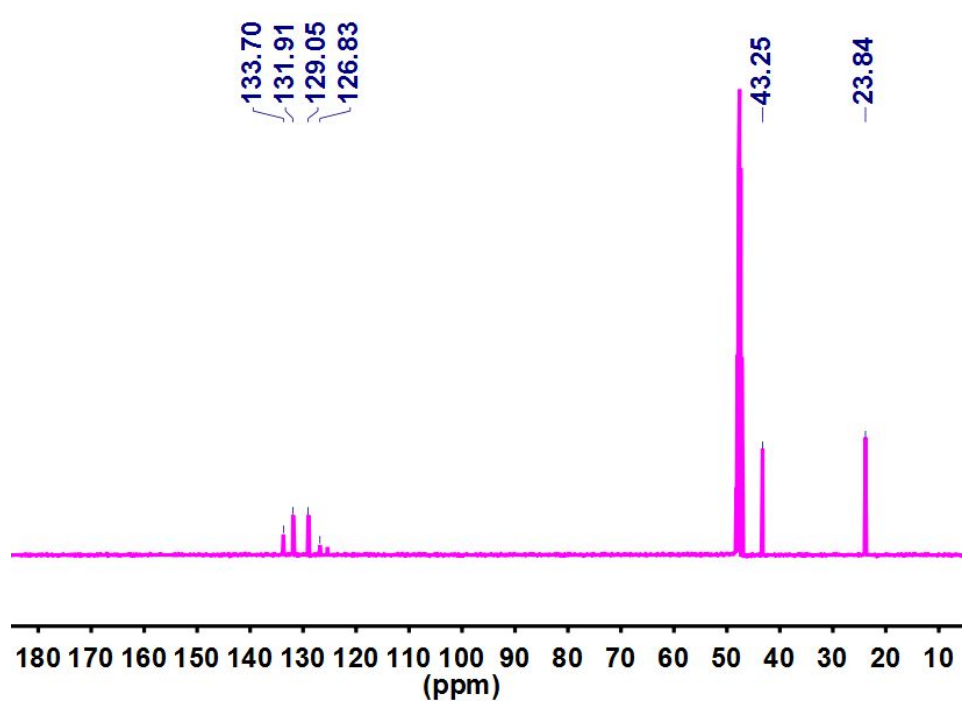

Figure $\mathrm{S} 13 .{ }^{13} \mathrm{C}$ NMR spectrum of PTAP.BF 4 


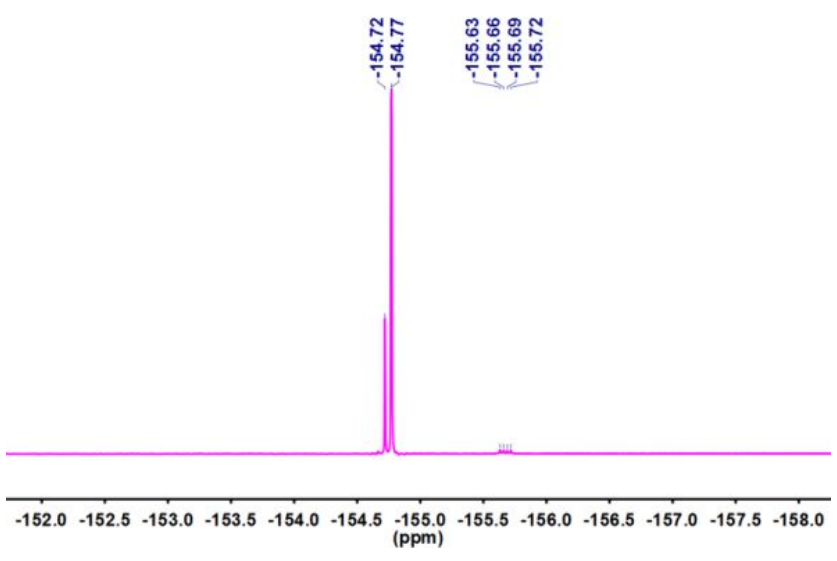

Figure S14. ${ }^{19} \mathrm{~F}$ NMR spectrum of PTAP $\cdot \mathrm{BF}_{4}$

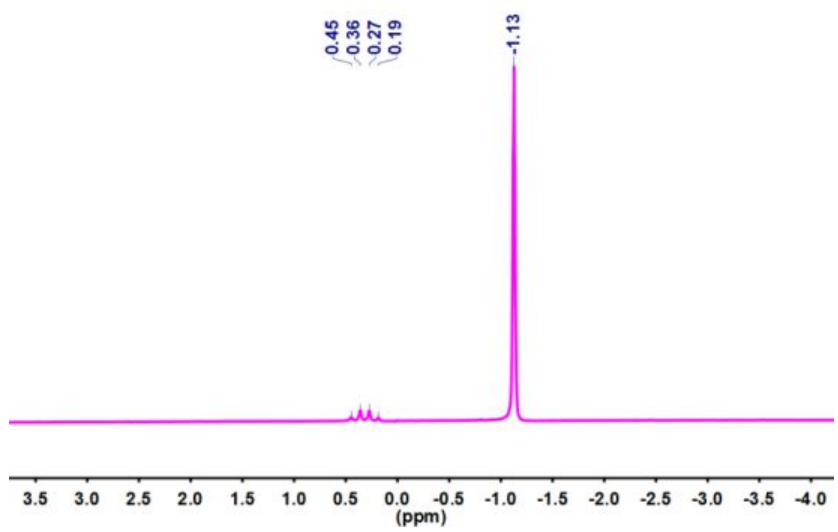

Figure S15. ${ }^{11} \mathrm{~B}$ NMR spectrum of PTAP $\cdot \mathrm{BF}_{4}$

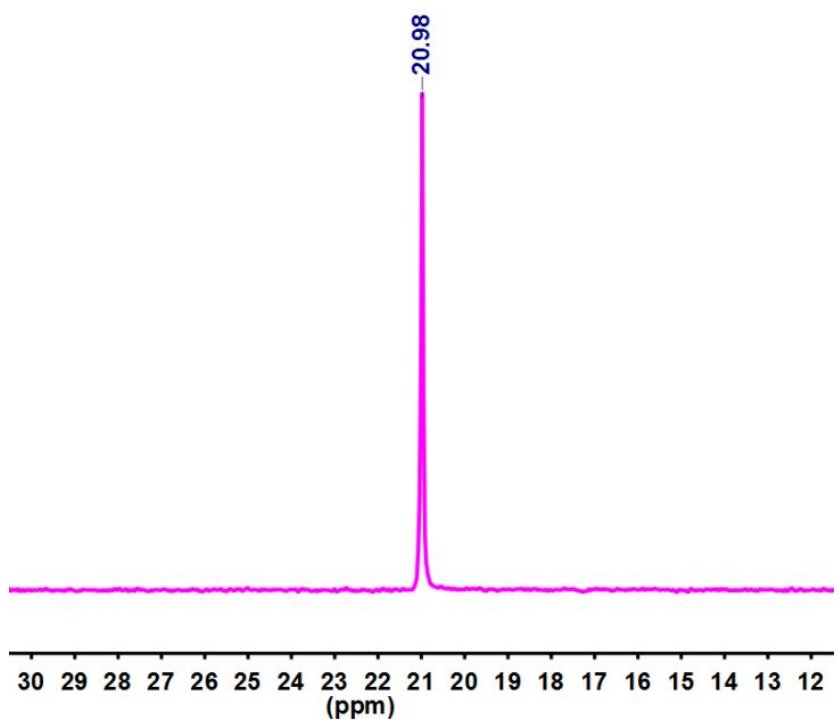

Figure S16. ${ }^{31} \mathrm{P}$ NMR spectrum of TIAP $\cdot \mathrm{BF}_{4}$ 


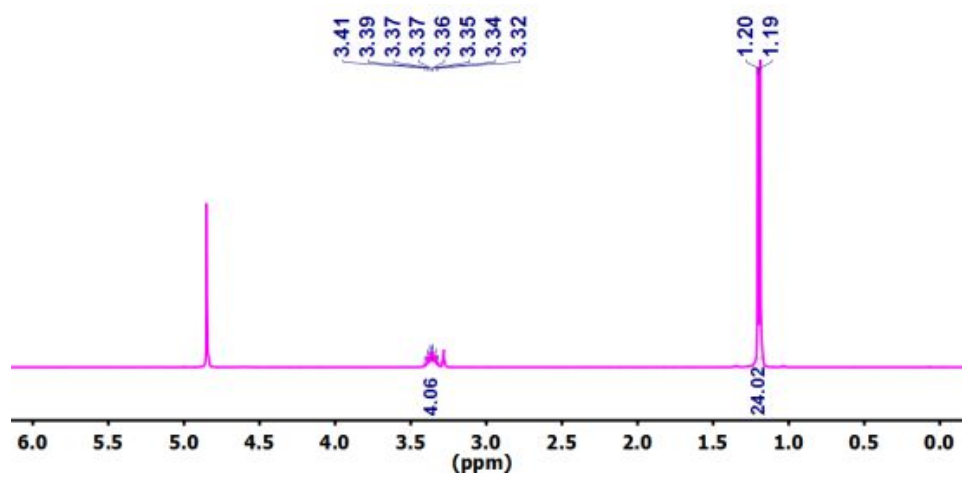

Figure S17. ${ }^{1} \mathrm{H}$ NMR spectrum of $\mathrm{TIAP}^{\mathrm{B}} \mathrm{BF}_{4}$

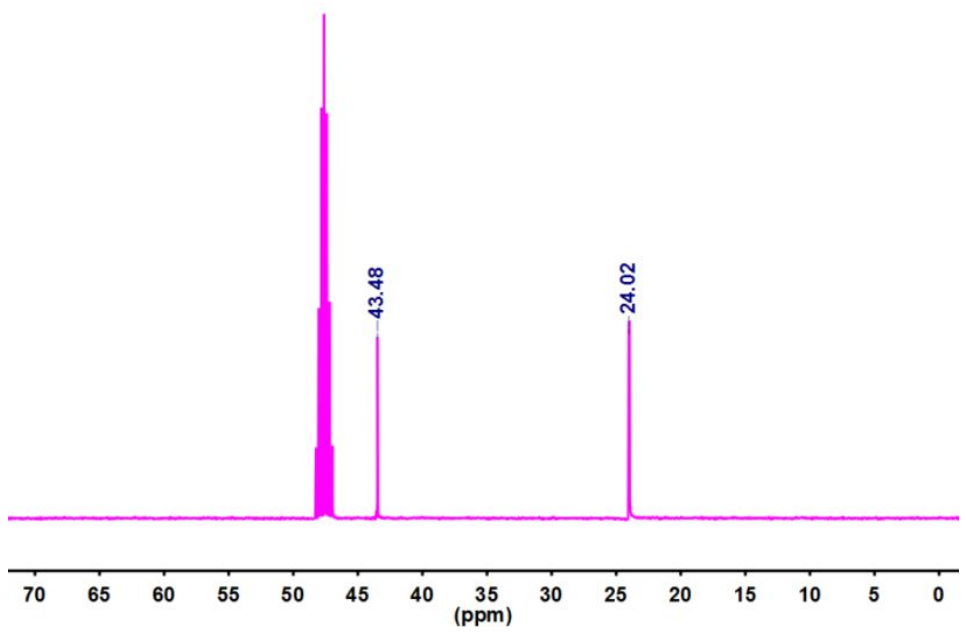

Figure S18. ${ }^{13} \mathrm{C}$ NMR spectrum of $\mathrm{TIAP} \cdot \mathrm{BF}_{4}$

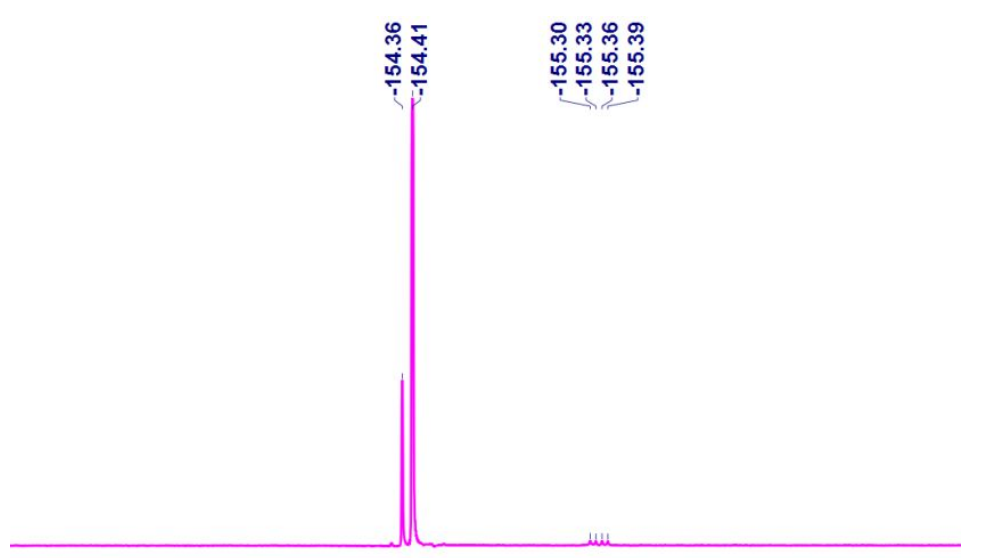

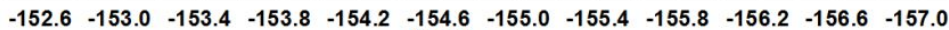
(ppm)

Figure S19. ${ }^{19} \mathrm{~F}$ NMR spectrum of $\mathrm{TIAP} \cdot \mathrm{BF}_{4}$ 


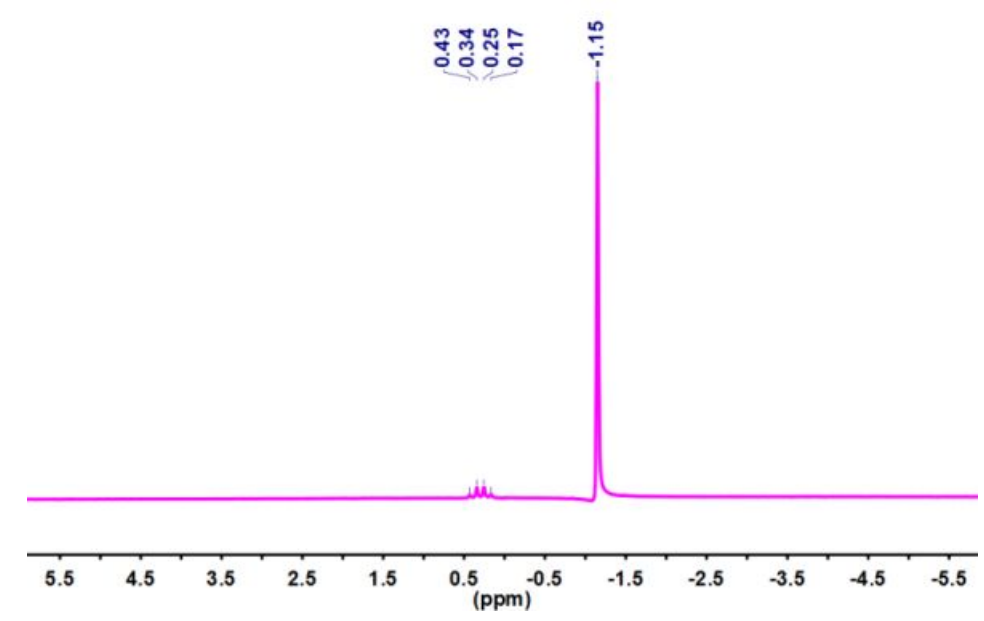

Figure S20. ${ }^{11} \mathrm{~B}$ NMR spectrum of TIAP.BF 4

(a)

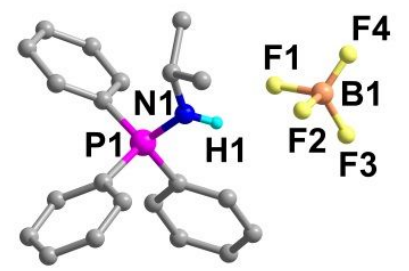

(b)

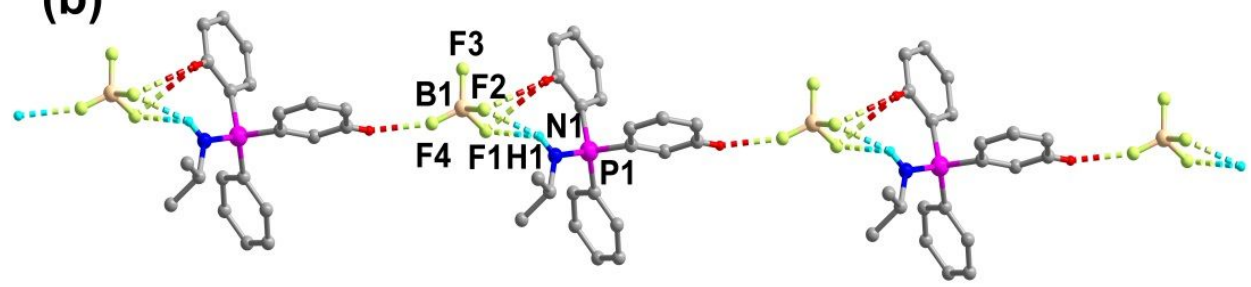

Figure S21. Molecular structure of TPAP $\cdot \mathrm{BF}_{4}$ at $100 \mathrm{~K}$ showing (a) its asymmetric unit and (b) H-bonding interactions along the 'b' axis.

(a)

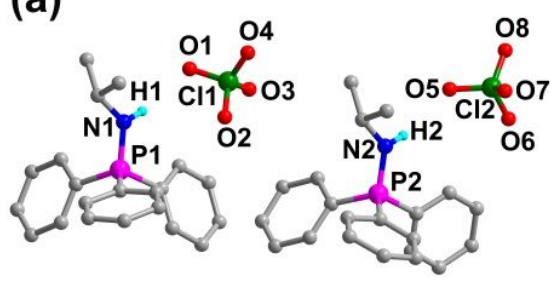

(c)

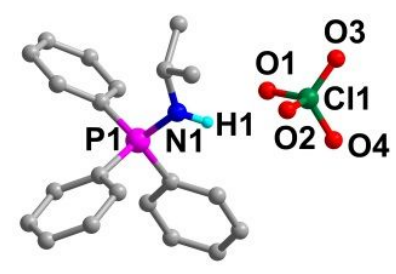

(b)

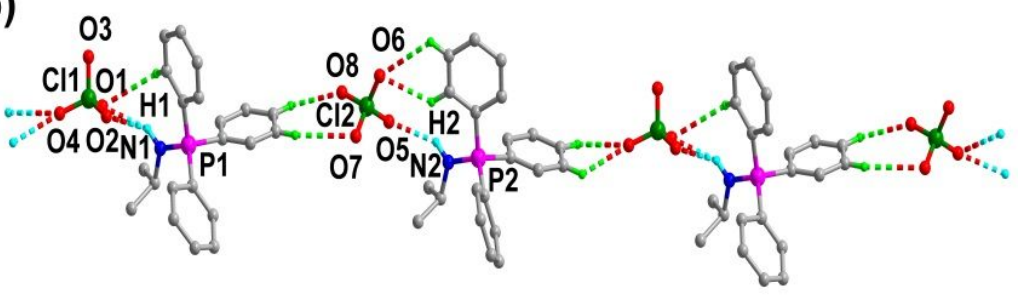

(d)

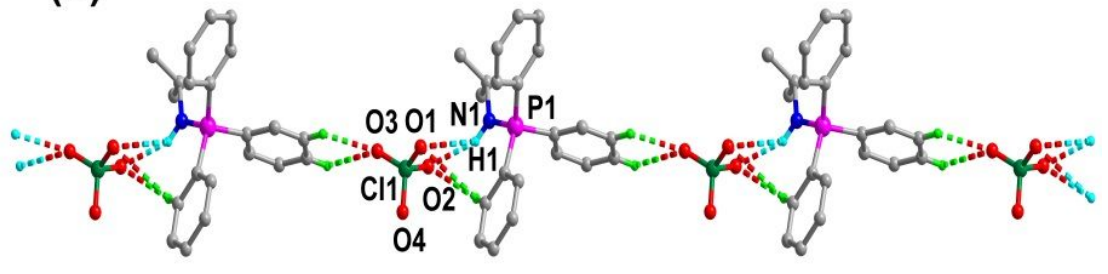

Figure S22. Molecular structure of TPAP. $\mathrm{ClO}_{4}$ at $150(\mathrm{a}, \mathrm{b})$ and 200 (c, d) $\mathrm{K}$ showing its asymmetric unit (a) and (c) and $\mathrm{H}$-bonding interactions along the 'b' axis (b) and (d). 
(a)

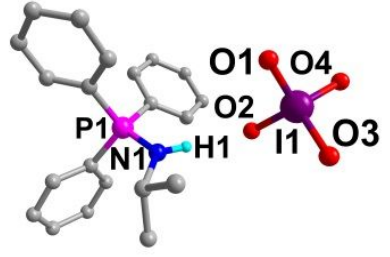

(b)

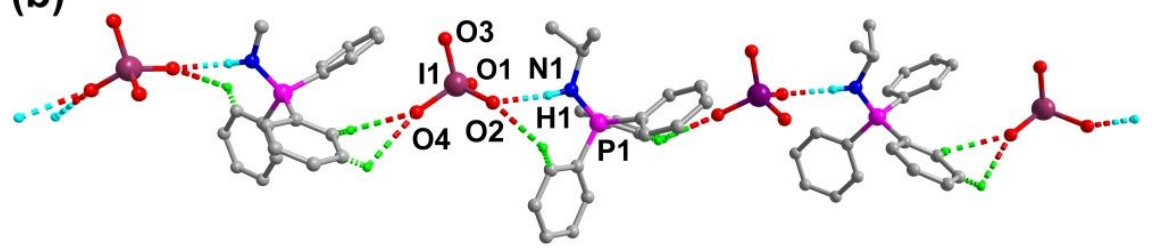

Figure S23. Molecular structure of TPAP. $1 \mathrm{O}_{4}$ at $100 \mathrm{~K}$ showing (a) its asymmetric unit and (b) $\mathrm{H}$-bonding interactions along the 'b' axis.

(a)

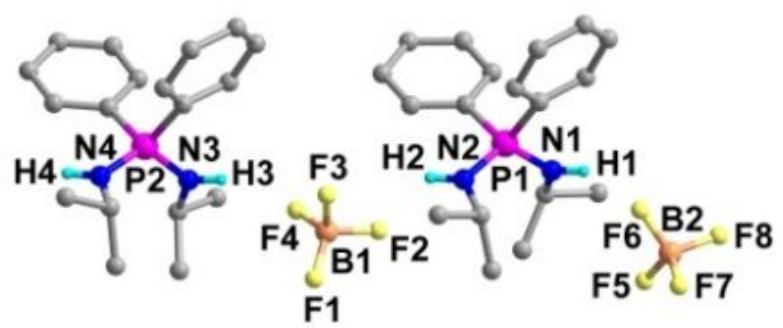

(b)

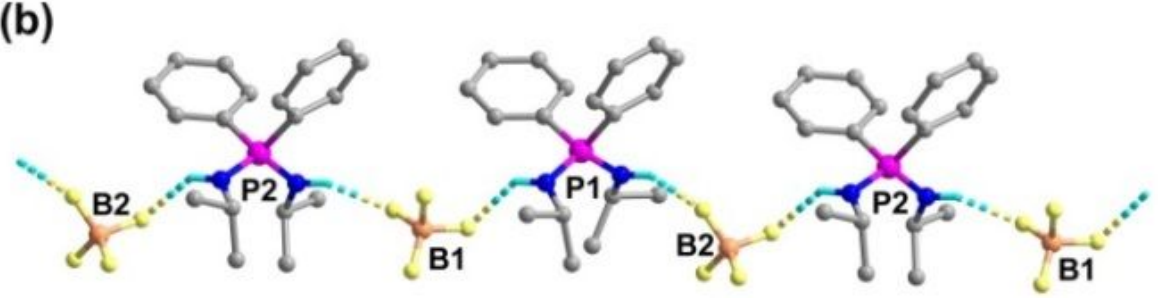

Figure S24. Molecular structure of DPDP.BF ${ }_{4}$ at $100 \mathrm{~K}$ showing (a) its asymmetric unit and (b) H-bonding interactions along the 'b' axis.

(a)
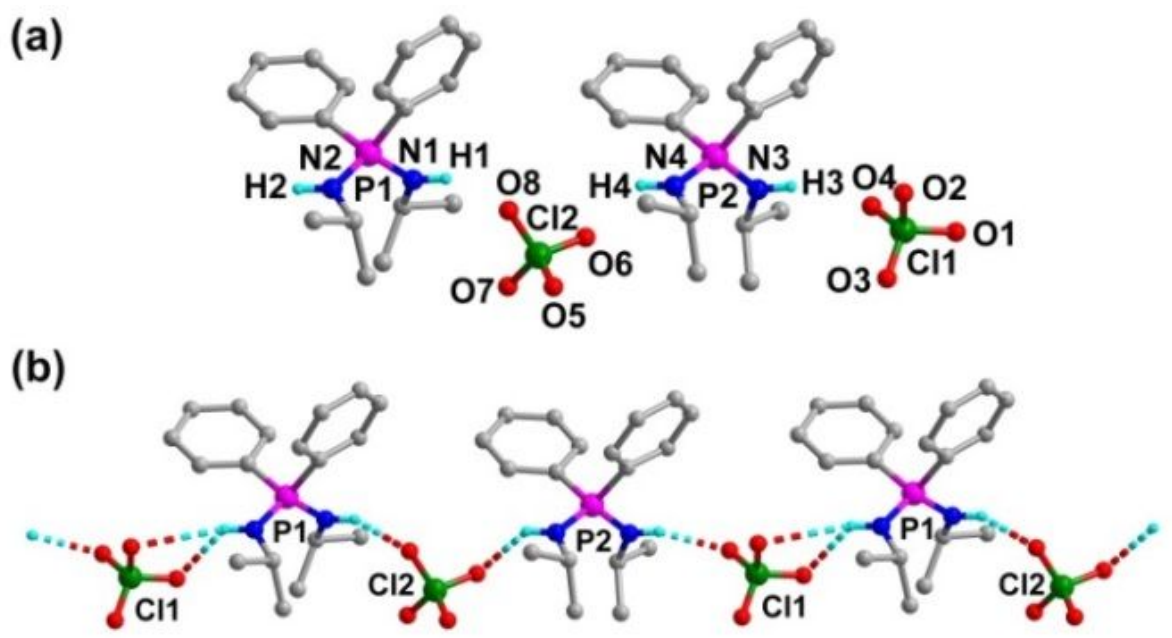

Figure S25. Molecular Structure of DPDP. $\mathrm{ClO}_{4}$ at $100 \mathrm{~K}$ showing (a) its asymmetric unit and (b) $\mathrm{H}$-bonding interactions along the 'b' axis. 
(a)

(b)
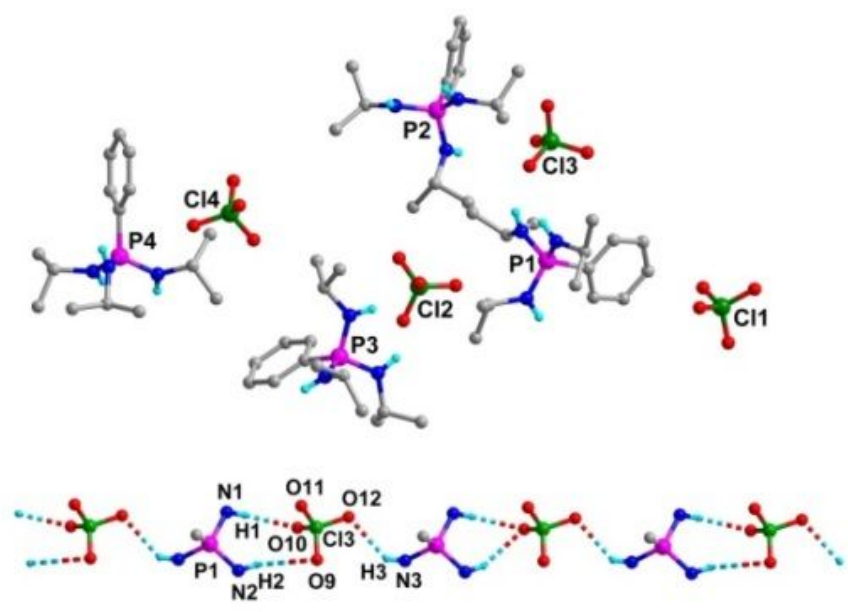

(c)

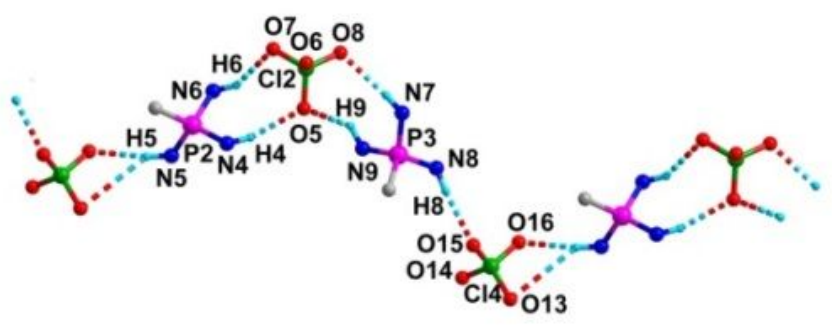

(d)

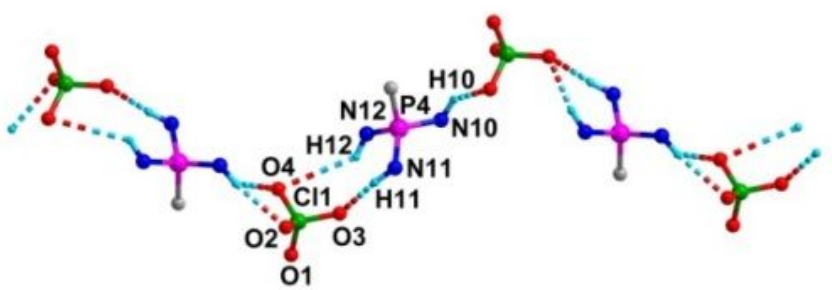

Figure S26. Molecular Structure of PTAP. $\mathrm{ClO}_{4}$ at $100 \mathrm{~K}$ along with its three different $\mathrm{H}$-bonded chains (a) its asymmetric unit (b)1D-chain like structure along the 'a' axis, (c) and (d) the Zig-Zag 1D-chains along the 'b' axis.

Phenyl and other alkyl groups are omitted for clarity.

(a)

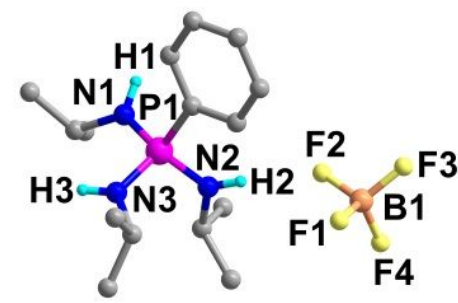

(b)

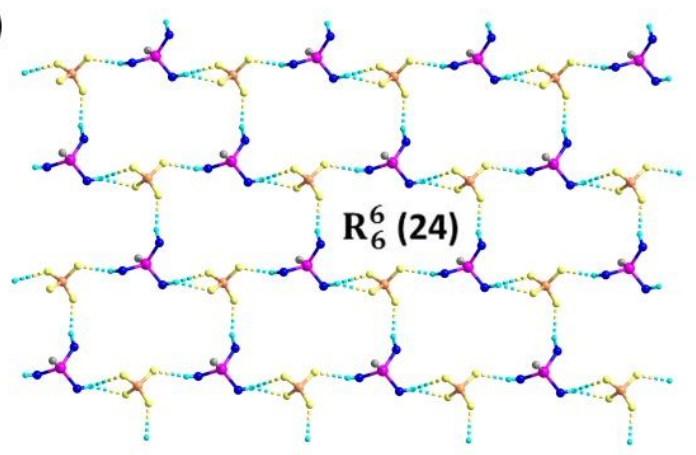

(c)

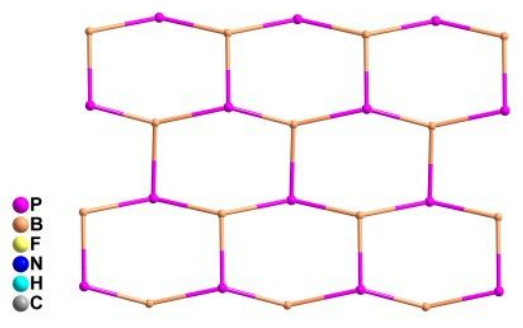

Figure S27. Molecular structure of PTAP.BF 4 at $100 \mathrm{~K}$ showing (a) its asymmetric unit and (b) formation of $2 \mathrm{D}$-sheet structure mediated by $\mathrm{N}-\mathrm{H}$...F interactions along with the view of a 24-membered macrocyclic ring present in it.

Phenyl and other alkyl groups are omitted for clarity. (c) The underlying distorted 2D-net of 'hcb' topology. 
(a)

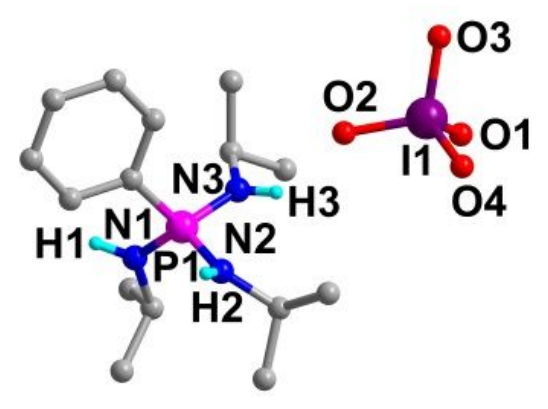

(b)

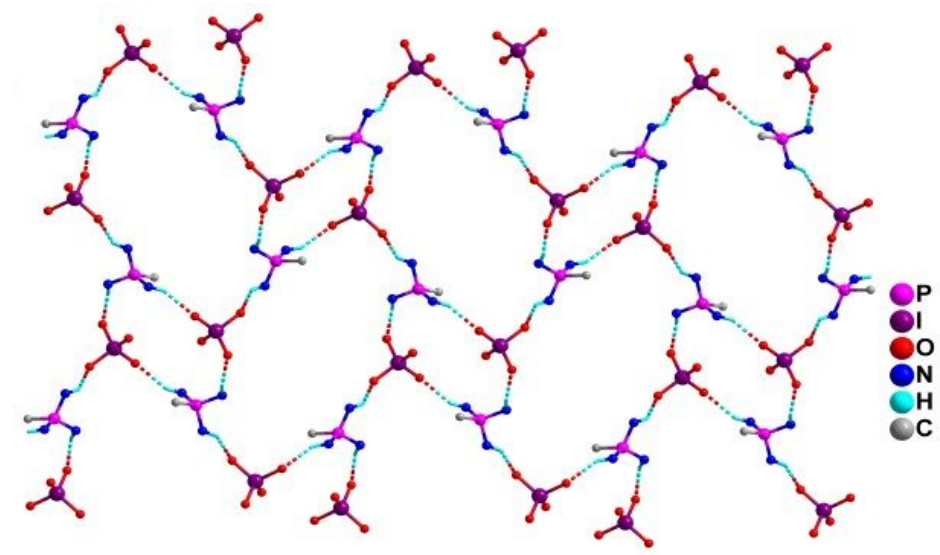

(e) (c)

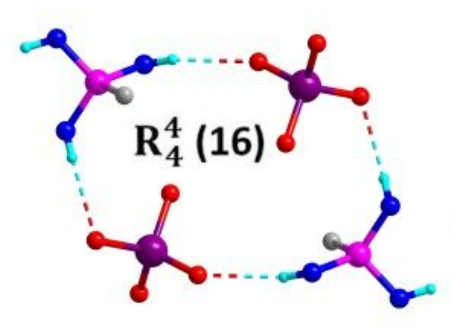

(d)

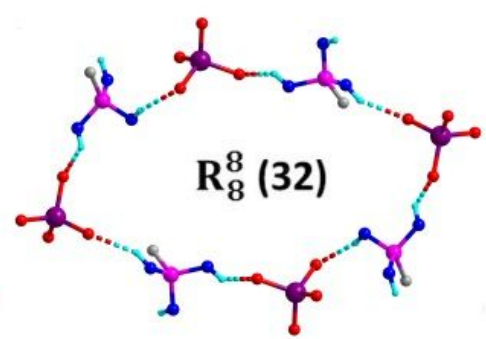

)

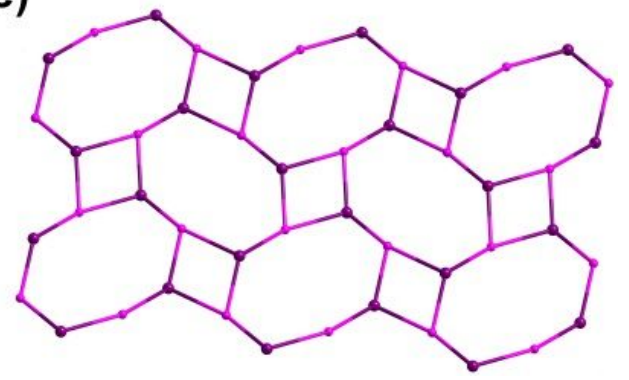

Figure S28. Molecular structure of PTAP $\cdot \mathrm{IO}_{4}$ at $100 \mathrm{~K}$ showing (a) its asymmetric unit and (b) 2D-hydrogen bonded networks viewed parallel to the 'bc' plane. (c) and (d) shows the respective individual 16 and 32 membered macrocycle rings. Phenyl and other alkyl groups are omitted for clarity. (e) The underlying 2D-net of 'fes' topology.

(a)

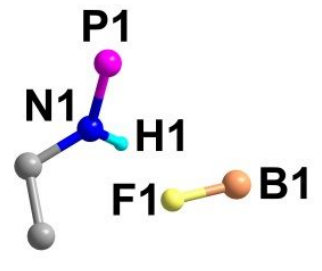

(c)

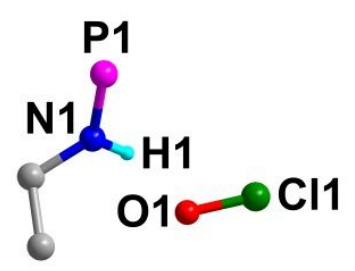

(b)

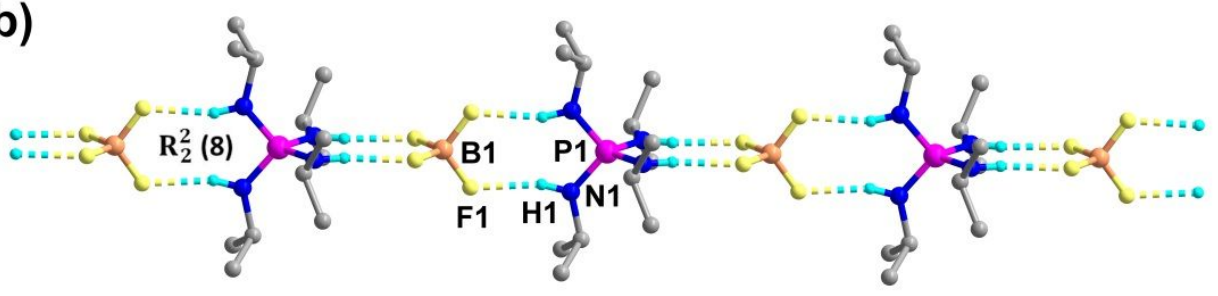

(d)

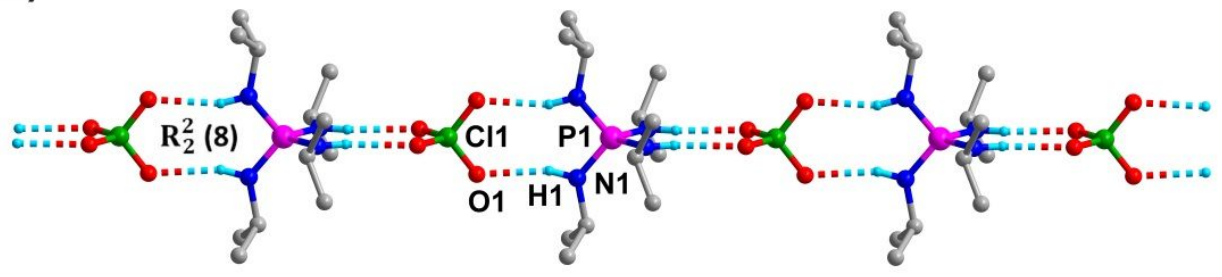

Figure S29. The asymmetric units present in the structures of (a) $\mathrm{TIAP}^{\mathrm{B}} \mathrm{BF}_{4}$ and (b) $\mathrm{TIAP} \cdot \mathrm{ClO}_{4}$ at $100 \mathrm{~K}$. (b) and (d) View of their iso-structural of $1 \mathrm{D}$-twisted hydrogen-bonded chains along 'c' axis. 


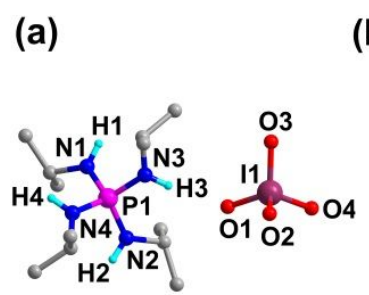

(b)

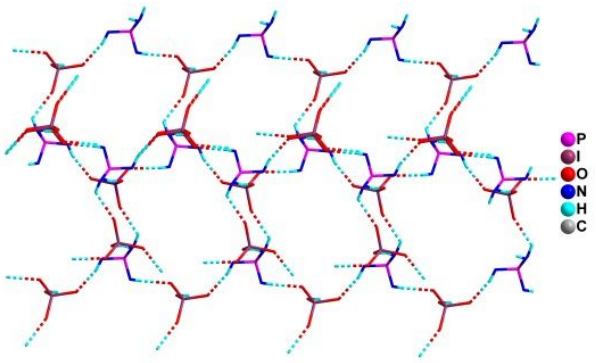

(c)

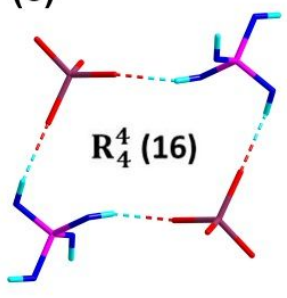

(f) (d)

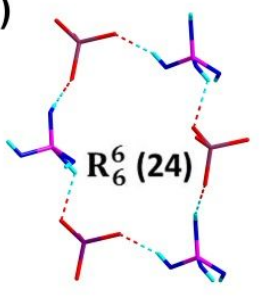

(g)

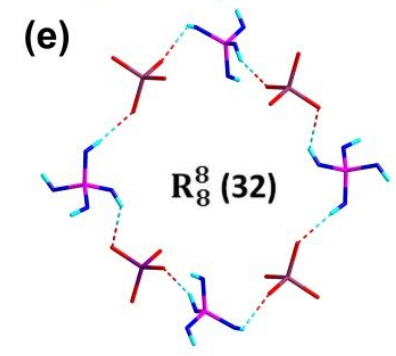

(e)

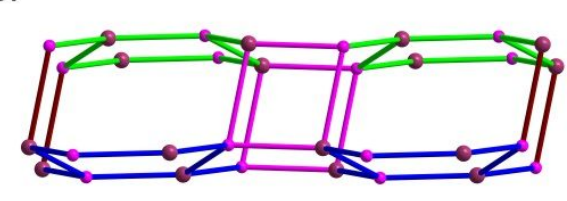

Figure S30. Molecular structure of TIAP $\cdot 1 \mathrm{I}_{4}$ at $100 \mathrm{~K}$ showing (a) its asymmetric unit and (b) the formation of the 3Dhydrogen bonded network in it. View of the (c) 16-, (d) 24- and (e) 32-membered macrocycle rings within the 3Dlattice. The alkyl groups are omitted for clarity. (f) The underlying 3D-net of 'crb' topology and (g) view of a single layer of 3D-network.

(a)

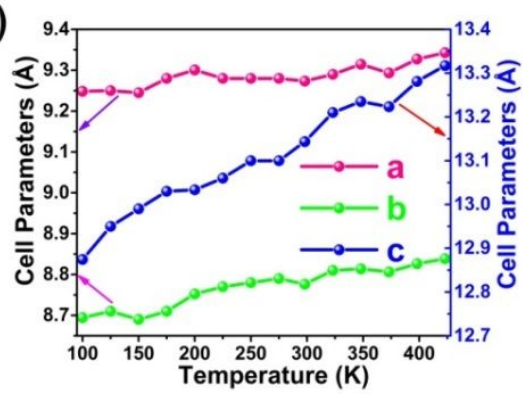

(b)

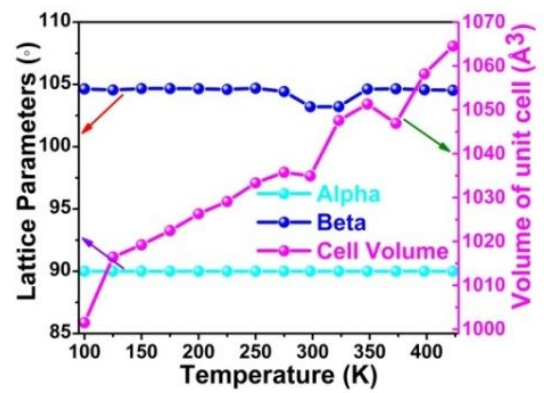

Figure S31. Variable temperature unit-cell parameters of $\mathrm{TPAP}^{\mathrm{B}} \mathrm{BF}_{4}$

(a)

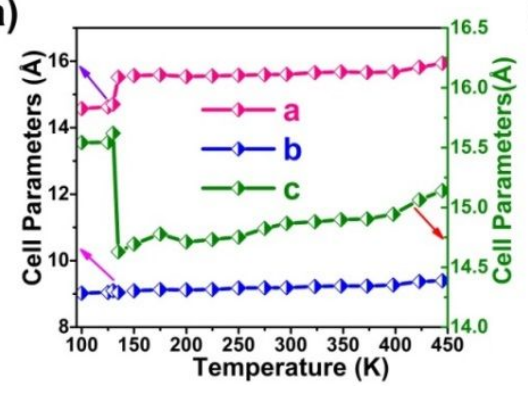

(b)

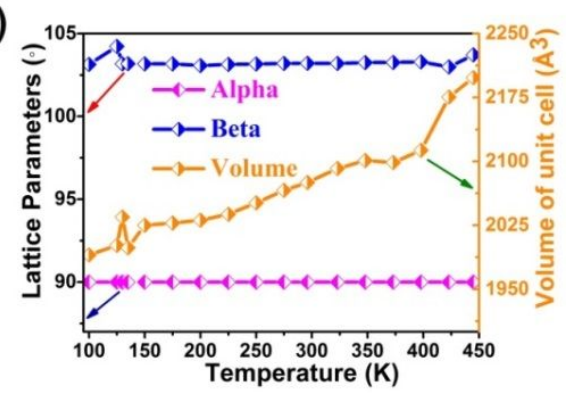

Figure S32. Variable temperature unit-cell parameters of $\mathrm{DPDP} \cdot \mathrm{BF}_{4}$ 
(a)

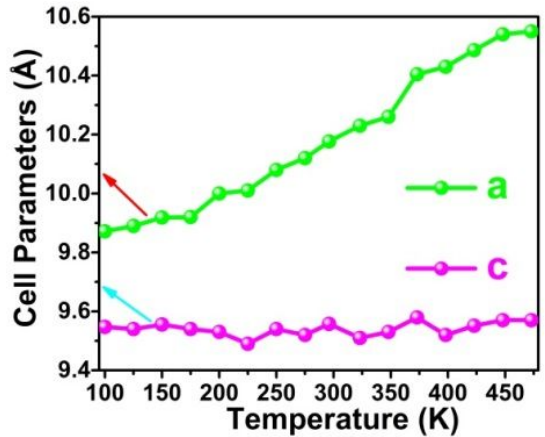

(b)

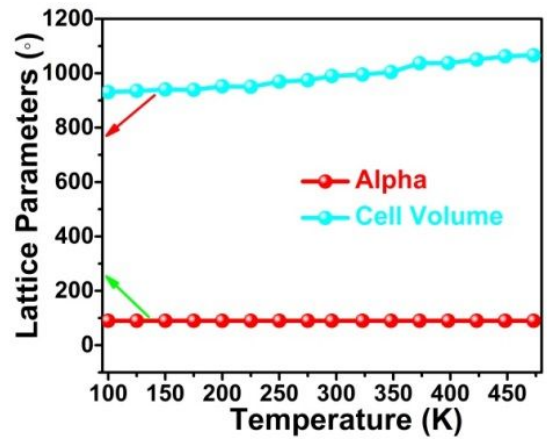

Figure S33. Variable temperature unit-cell parameters of TIAP $\cdot \mathrm{BF}_{4}$

(a)

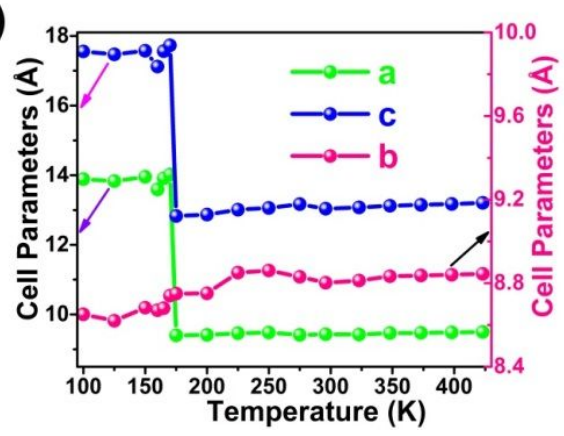

(b)

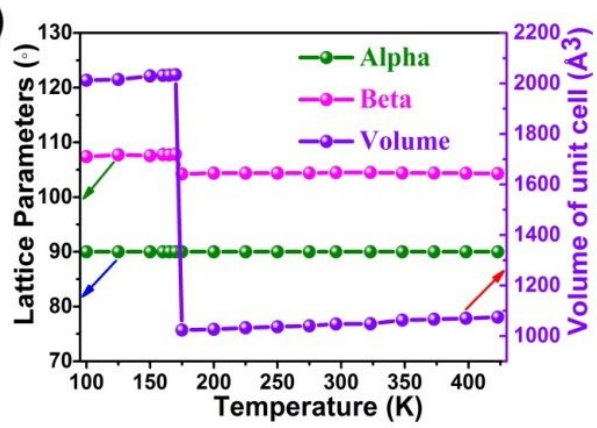

Figure S34. Variable temperature unit-cell parameters of TPAP $\cdot \mathrm{ClO}_{4}$

(a)

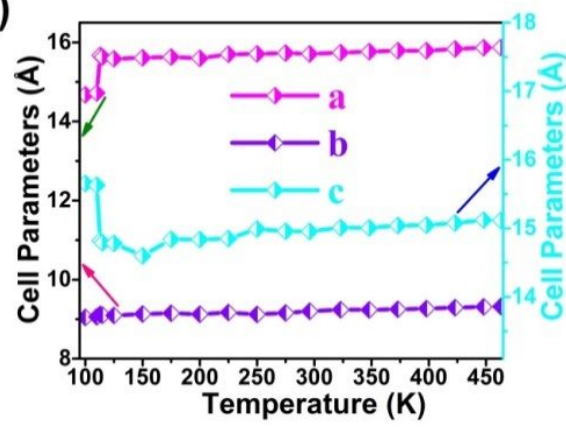

(b)

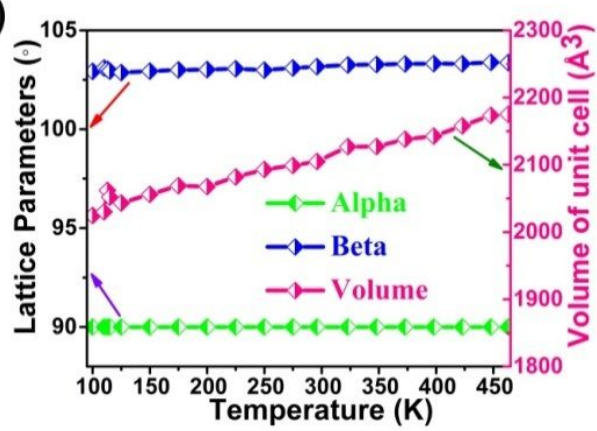

Figure S35. Variable temperature unit-cell parameters of DPDP. $\mathrm{ClO}_{4}$

(a)

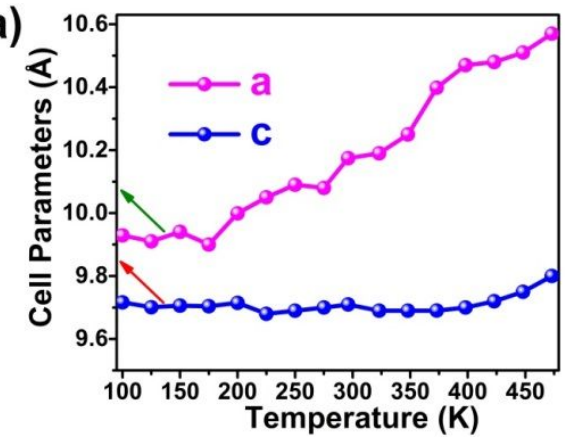

(b)

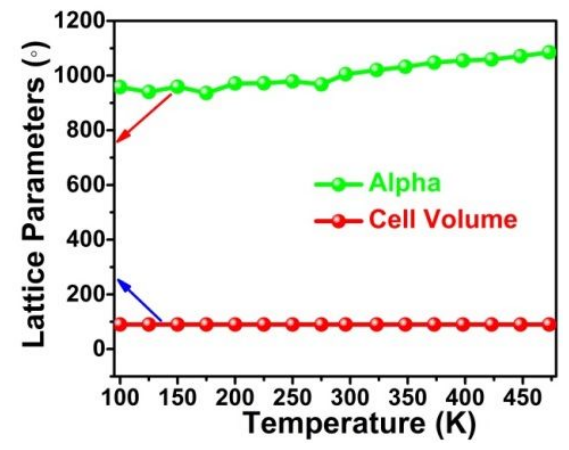

Figure S36. Variable temperature unit-cell parameters of TIAP $\cdot \mathrm{ClO}_{4}$ 
(a)

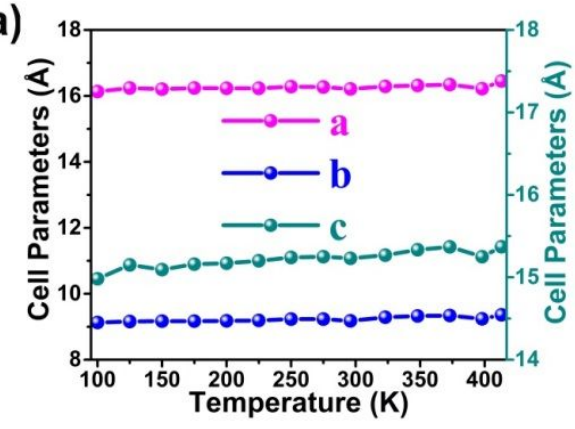

(b)

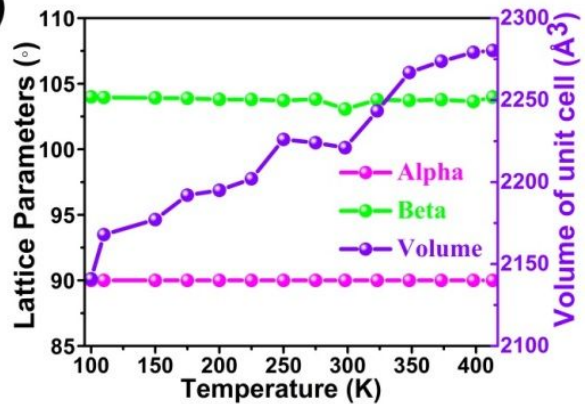

Figure S37. Variable temperature unit-cell parameters of DPDP.IO

(a)

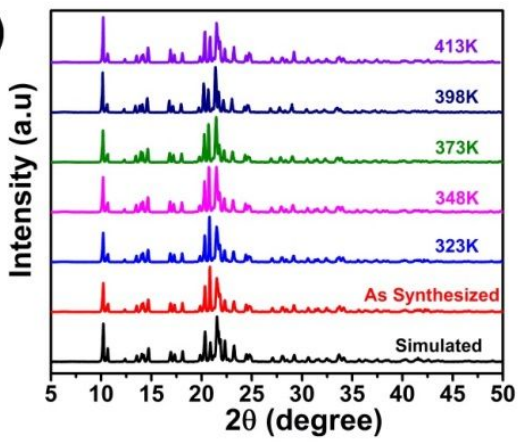

(b)

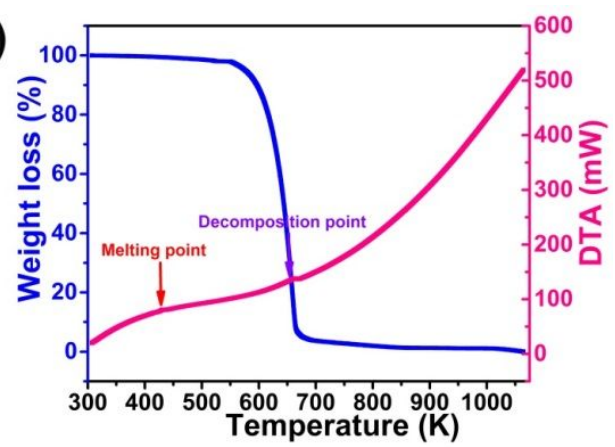

Figure S38. VT-PXRD, TG/DTA analysis of TPAP.BF 4

(a)

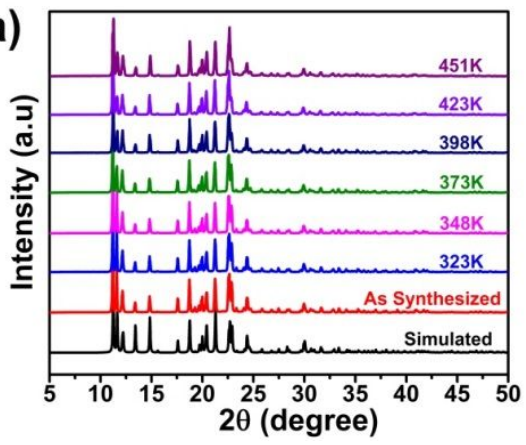

(b)

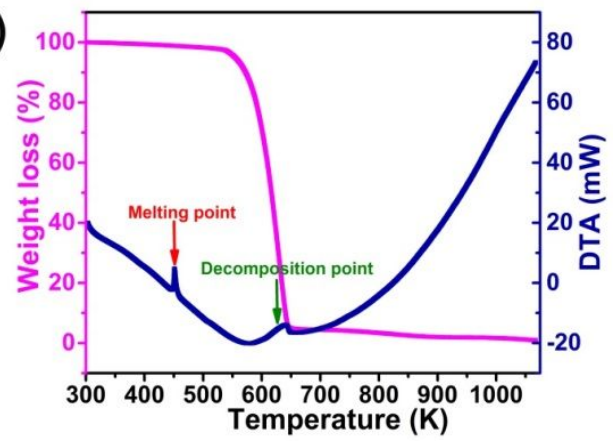

Figure S39. VT-PXRD, TG/DTA analysis of DPDP.BF 4

(a)

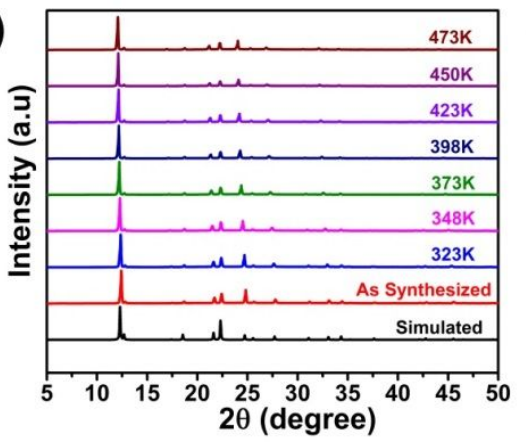

(b)

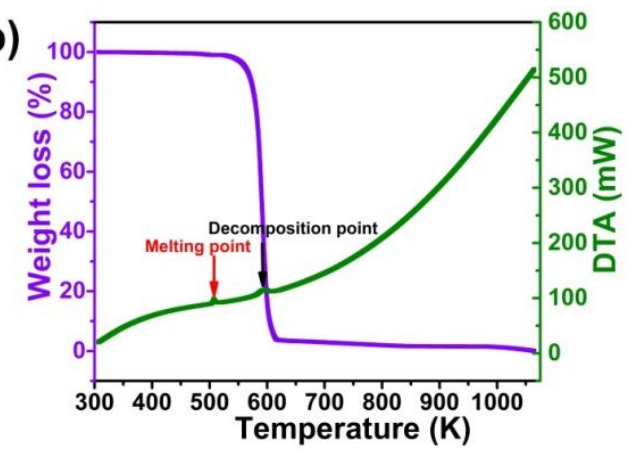

Figure S40. VT-PXRD, TG/DTA analysis of TIAP.BF 4 
(a)

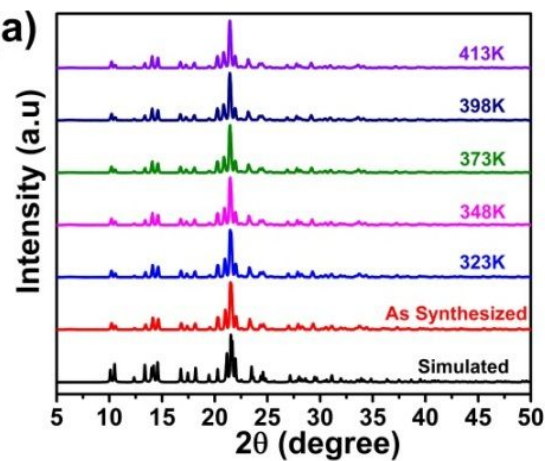

(c)

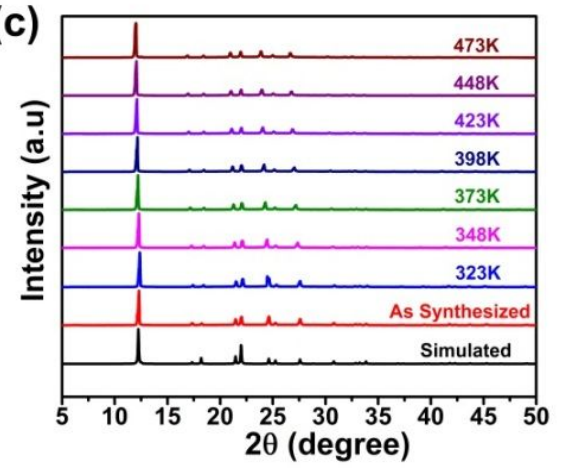

(b)

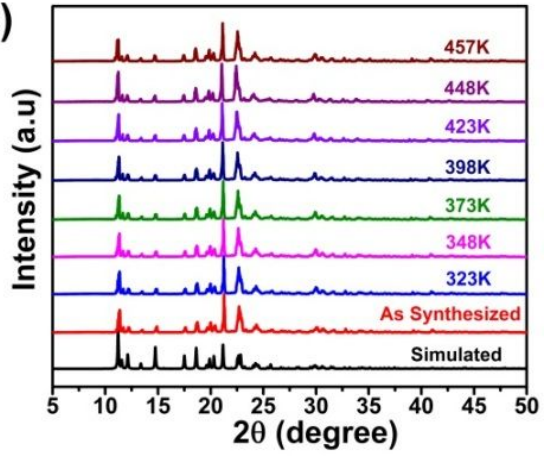

(d)

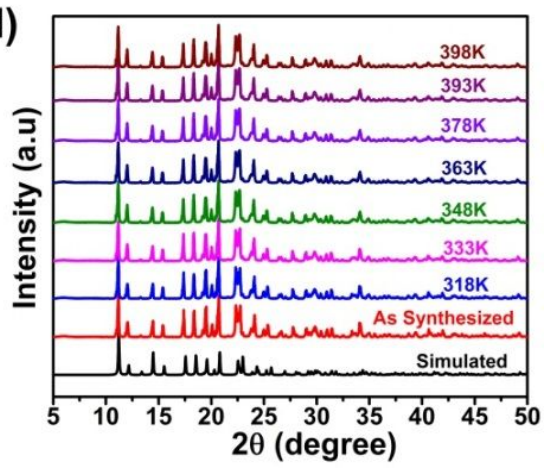

Figure S41. VT-PXRD pattern of (a) TPAP $\cdot \mathrm{ClO}_{4}$, (b) DPDP $\cdot \mathrm{ClO}_{4}$, (c) TIAP.ClO ${ }_{4}$ and (d) DPDP.IO

Table S5. SHG measurements of all the newly synthesized phosphonium salts supported by tetrahedral anions

\begin{tabular}{|c|c|c|}
\hline S.No & $\begin{array}{c}\text { Ferro and Piezoelectric } \\
\text { Materials }\end{array}$ & $\begin{array}{l}\text { SHG efficiency in } \\
(\mathrm{mV})\end{array}$ \\
\hline 1 & KDP & 24 \\
\hline 2 & TPAP·BF 4 & 6 \\
\hline 3 & $\mathrm{DPDP} \cdot \mathrm{BF}_{4}$ & 28 \\
\hline 4 & TIAP-BF 4 & 6 \\
\hline 5 & $\mathrm{TPAP} \cdot \mathrm{CIO}_{4}$ & 7 \\
\hline 6 & $\mathrm{DPDP} \cdot \mathrm{CIO}_{4}$ & 7 \\
\hline 7 & $\mathrm{TIAP} \cdot \mathrm{ClO}_{4}$ & 4 \\
\hline 8 & DPDP.IO ${ }_{4}$ & 3 \\
\hline
\end{tabular}
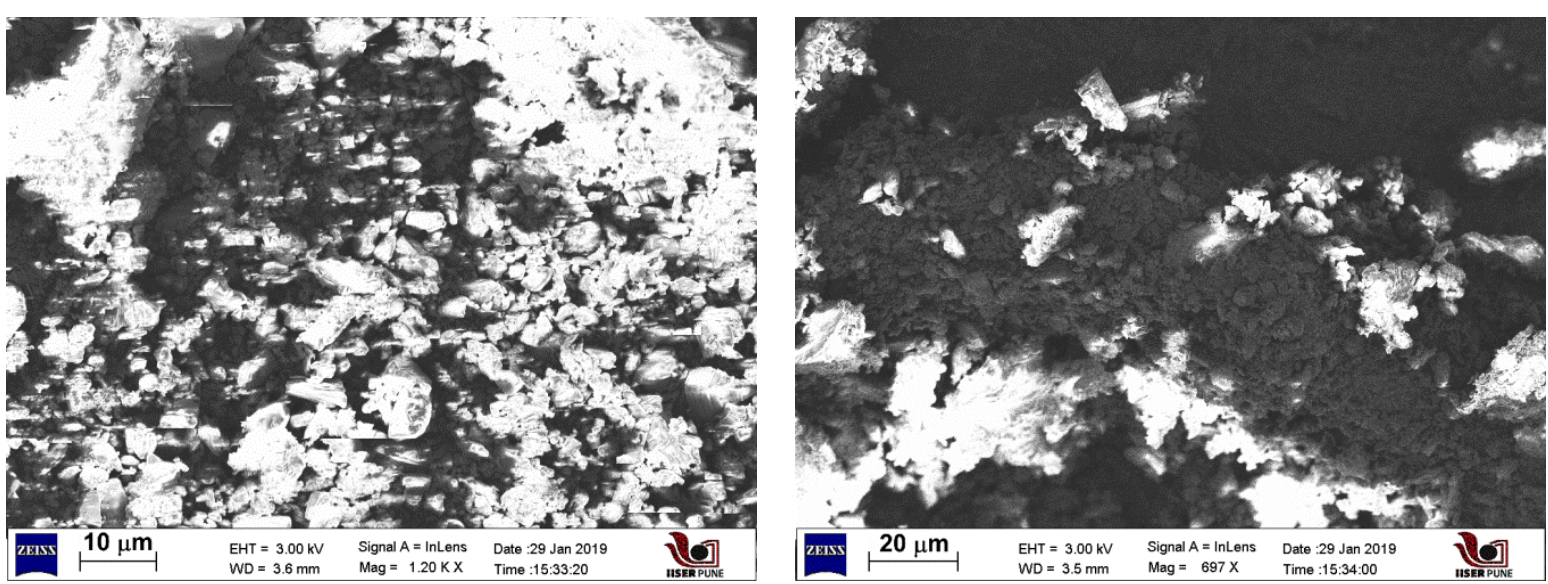

Figure S42. SEM images of TPAP·BF 4 

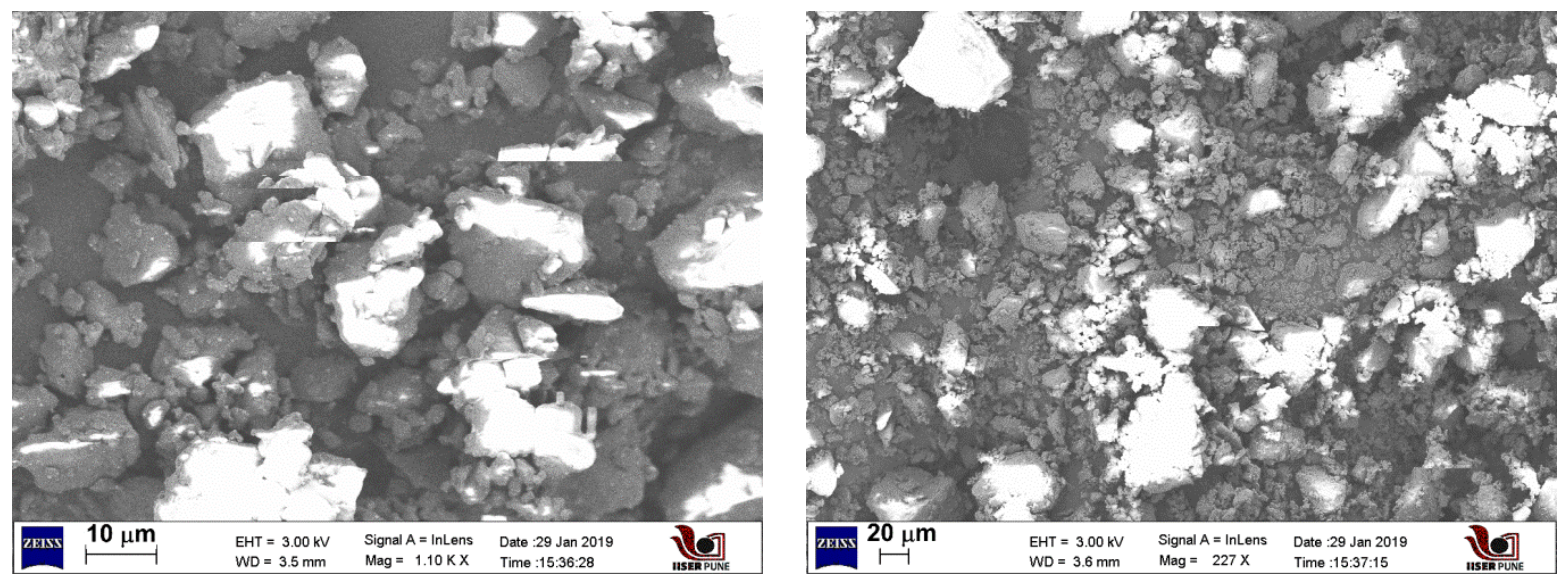

Figure S43. SEM images of DPDP. $\mathrm{BF}_{4}$
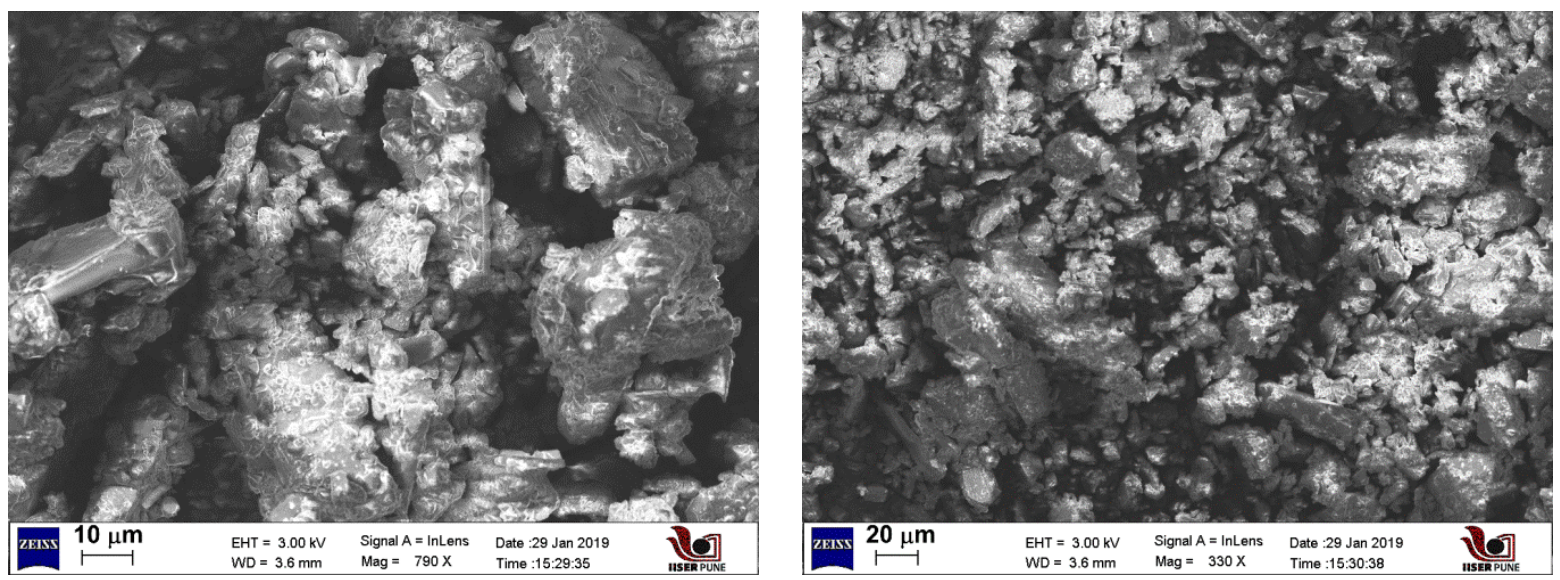

Figure S44. SEM images of TIAP.BF 4
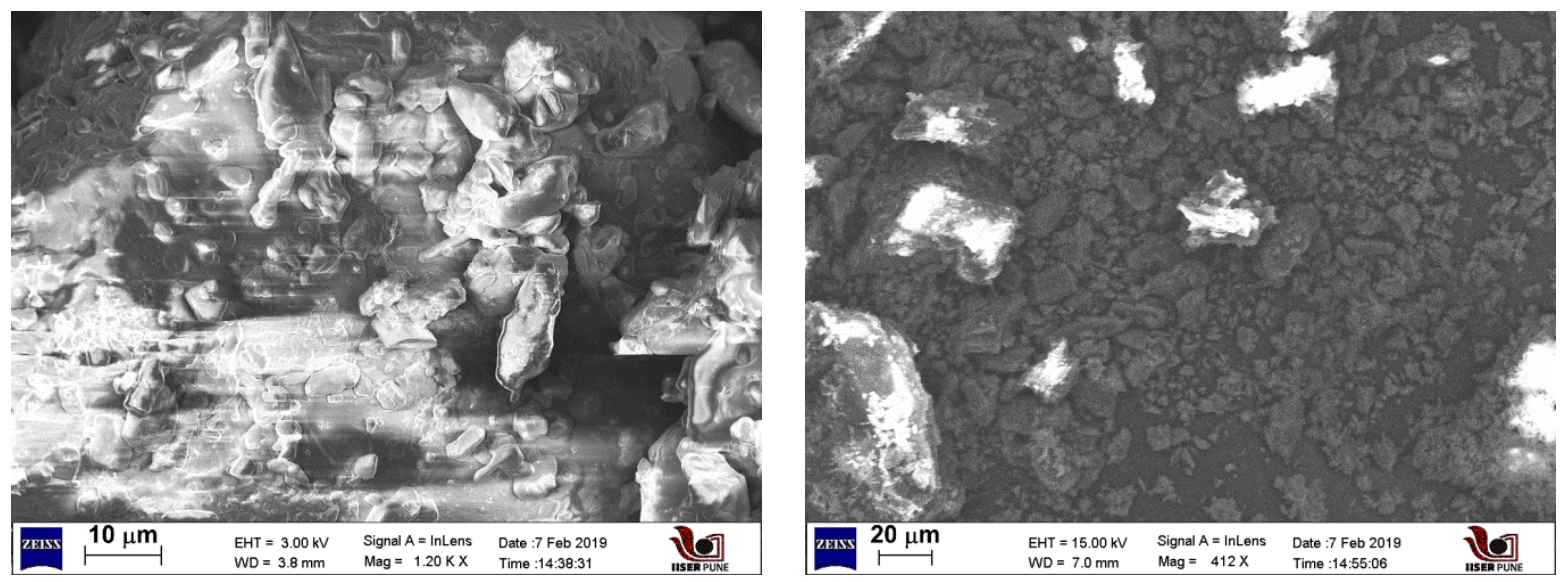

Figure S45. SEM images of TPAP. $\mathrm{ClO}_{4}$ 

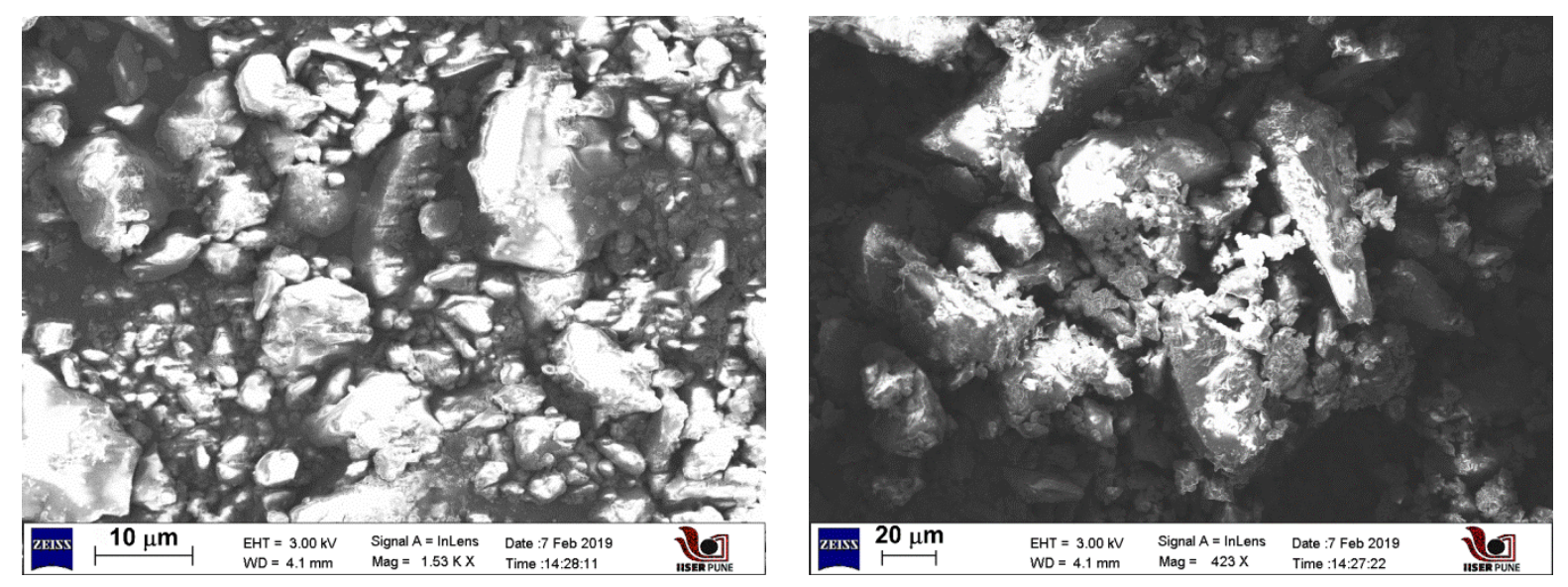

Figure S46. SEM images of DPDP. $\mathrm{ClO}_{4}$
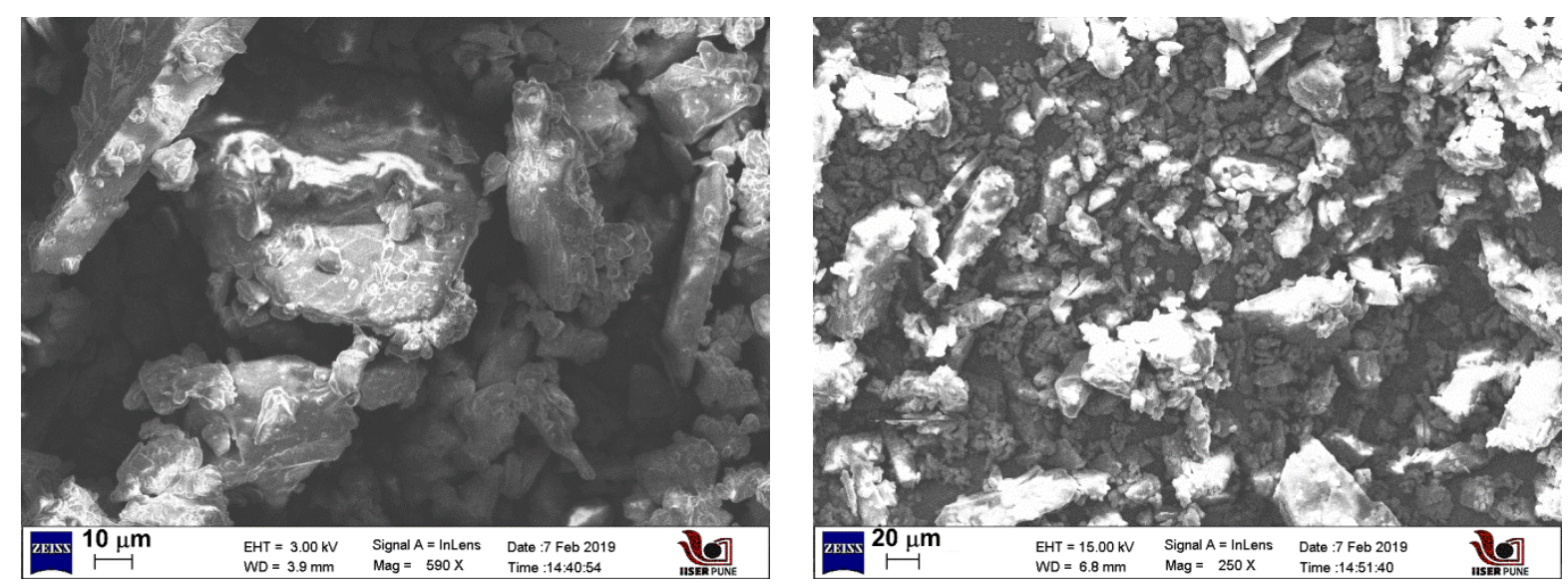

Figure S47. SEM images of TIAP. $\mathrm{CIO}_{4}$
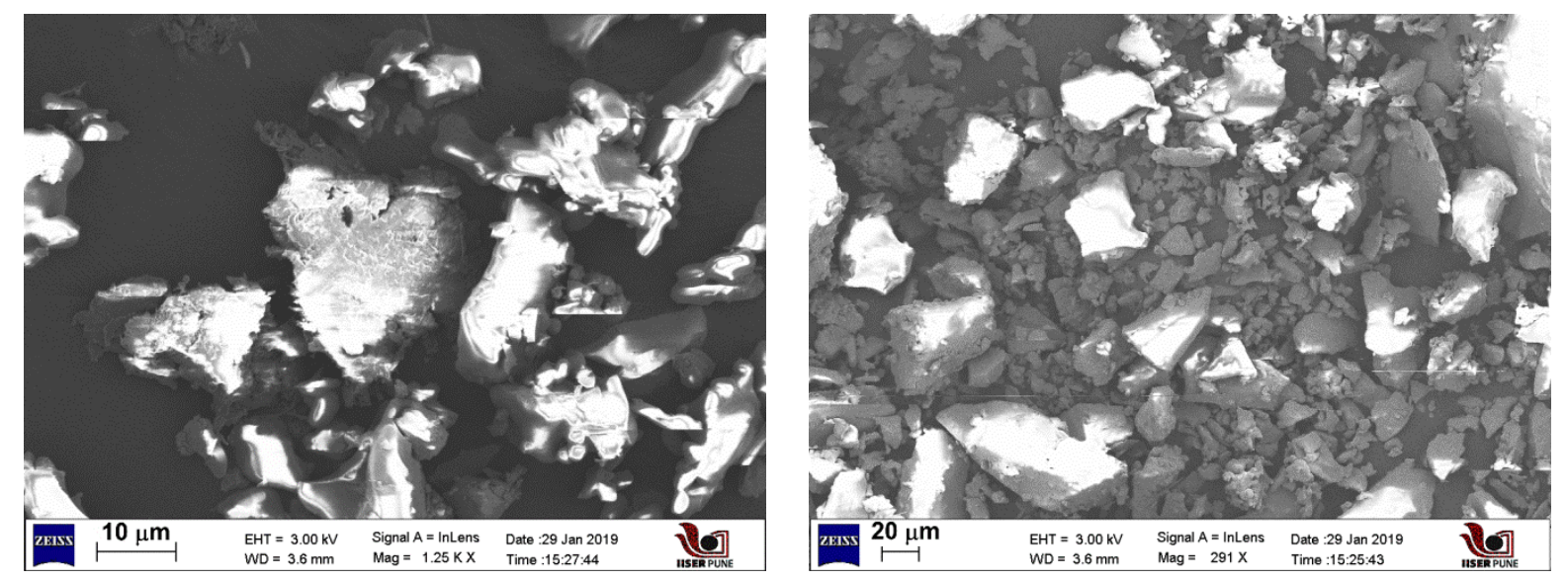

Figure S48. SEM images of DPDP.1O 


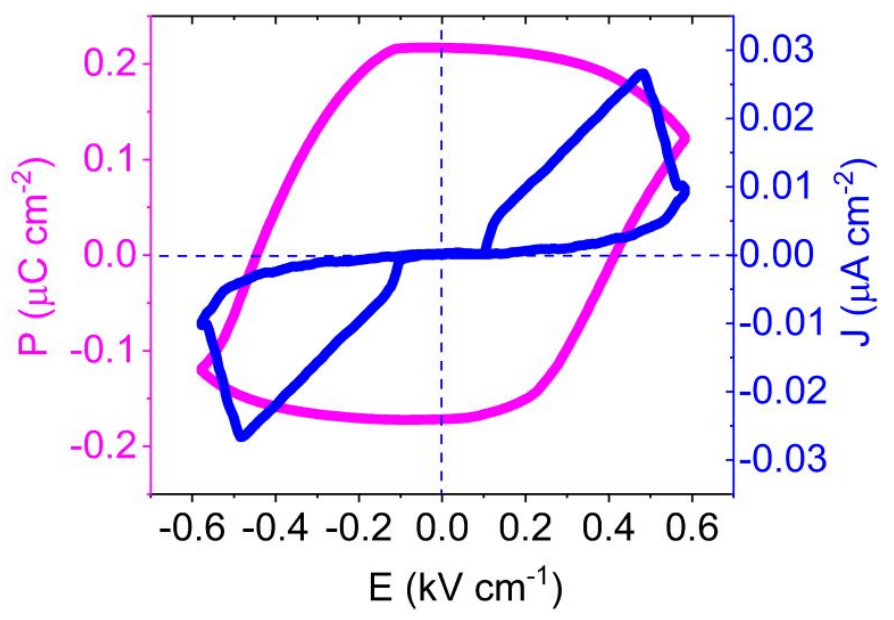

Figure S49. The $P$-E hysteresis loop and leakage current density plots of TPAP.BF 4

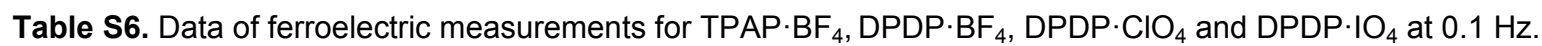

\begin{tabular}{|c|c|c|c|c|c|c|}
\hline S.No & $\begin{array}{c}\text { Ferroelectric } \\
\text { Materials }\end{array}$ & $\begin{array}{c}\text { Area } \\
\left(\mathrm{mm}^{2}\right)\end{array}$ & $\begin{array}{c}\text { Thickness } \\
\text { (nm) }\end{array}$ & $P_{r}\left(\mu C \mathrm{~cm}^{-2}\right)$ & $E_{c}\left(k V c m^{-1}\right)$ & $\begin{array}{c}\text { Current } \\
\text { range }\end{array}$ \\
\hline 1 & TPAP $\cdot \mathrm{BF}_{4}$ & 9.576 & $1.37 \mathrm{e}^{6}$ & 0.22 & 0.42 & $100 \mathrm{nA}$ \\
\hline 2 & DPDP.BF 4 & 13 & $8 e^{5}$ & 35.36 & 1.38 & $100 \mu \mathrm{A}$ \\
\hline 3 & DPDP. $\mathrm{ClO}_{4}$ & 8.87 & $1.27 e^{6}$ & 21.83 & 0.87 & $100 \mu \mathrm{A}$ \\
\hline 4 & DPDP:IO & 8.9 & $2.18 \mathrm{e}^{6}$ & 21.12 & 2.36 & $10 \mu \mathrm{A}$ \\
\hline
\end{tabular}

(a)

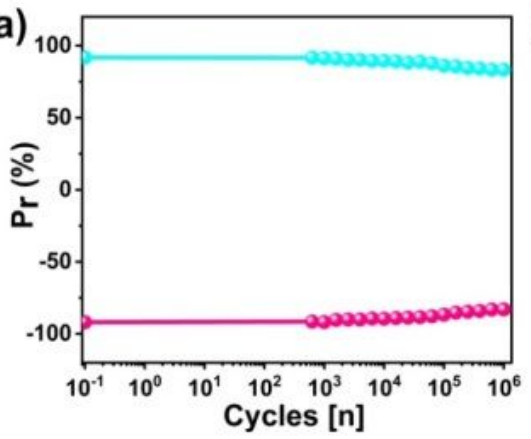

(c)

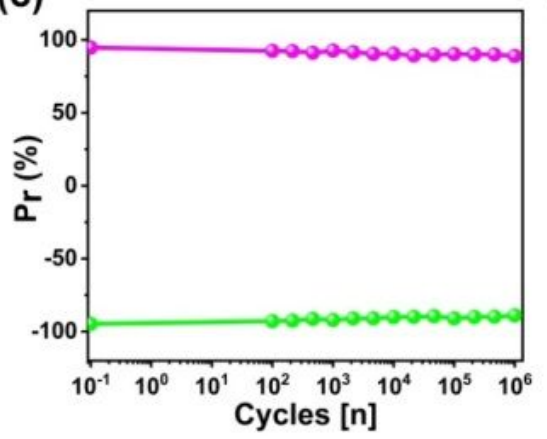

(b)

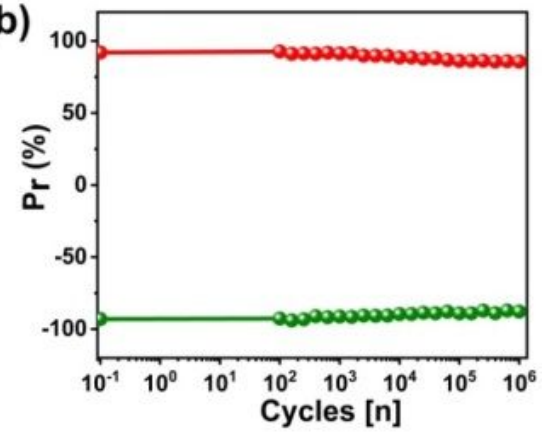

(d)

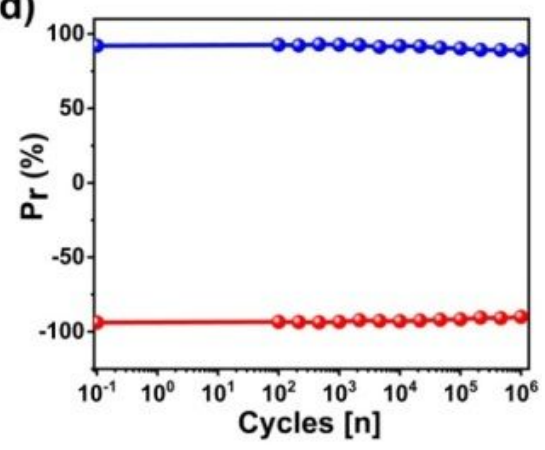

Figure S50. Ferroelectric fatigue data for (a) TPAP·BF 

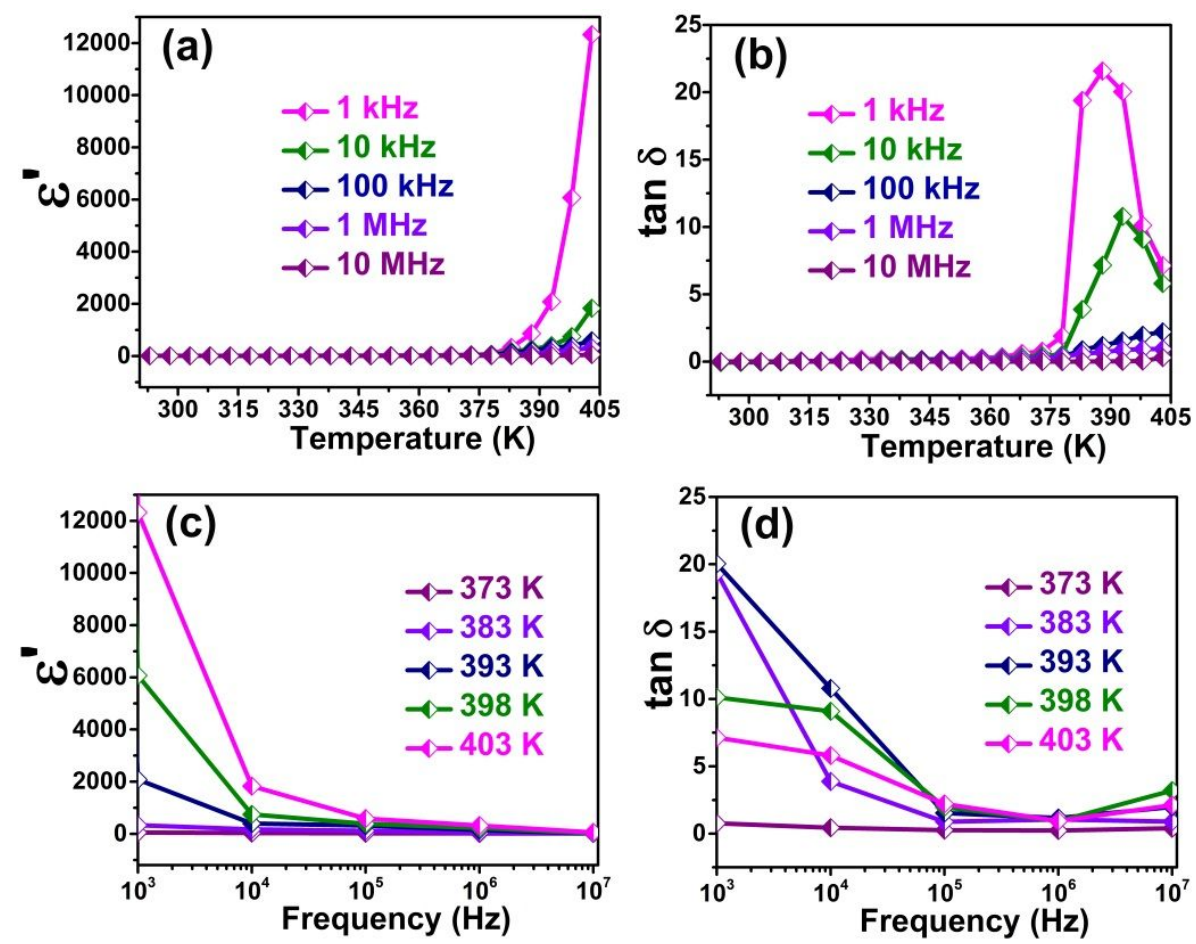

Figure S51. ( $a$ and b) temperature and (c and d) frequency dependent dielectric constant $\left(\varepsilon^{\prime}\right)$ and dielectric loss (tanס) of TPAP.BF 4
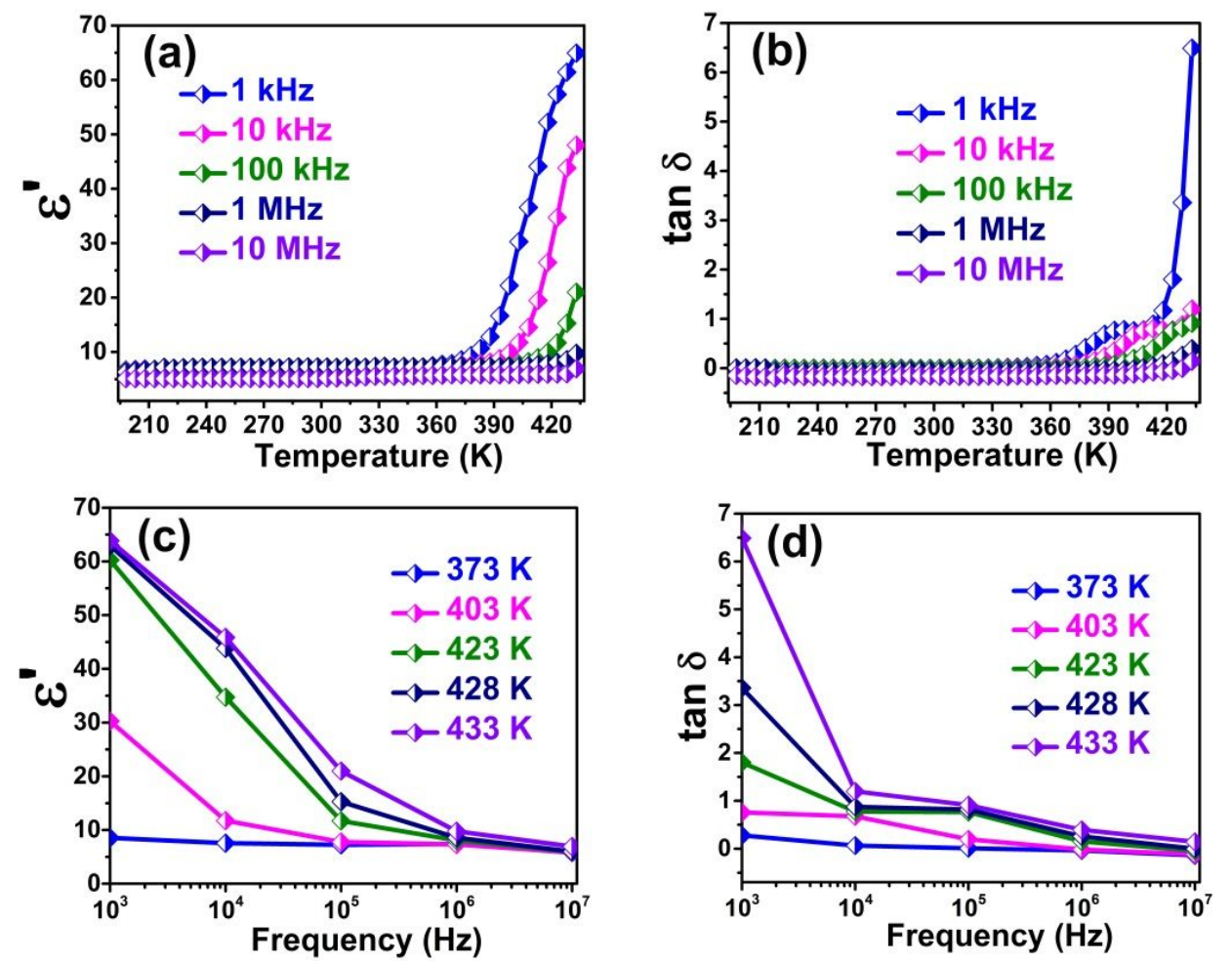

Figure S52. ( $a$ and b) temperature and (c and d) frequency dependent $\varepsilon^{\prime}$ and tan $\delta$ of DPDP·BF 4 

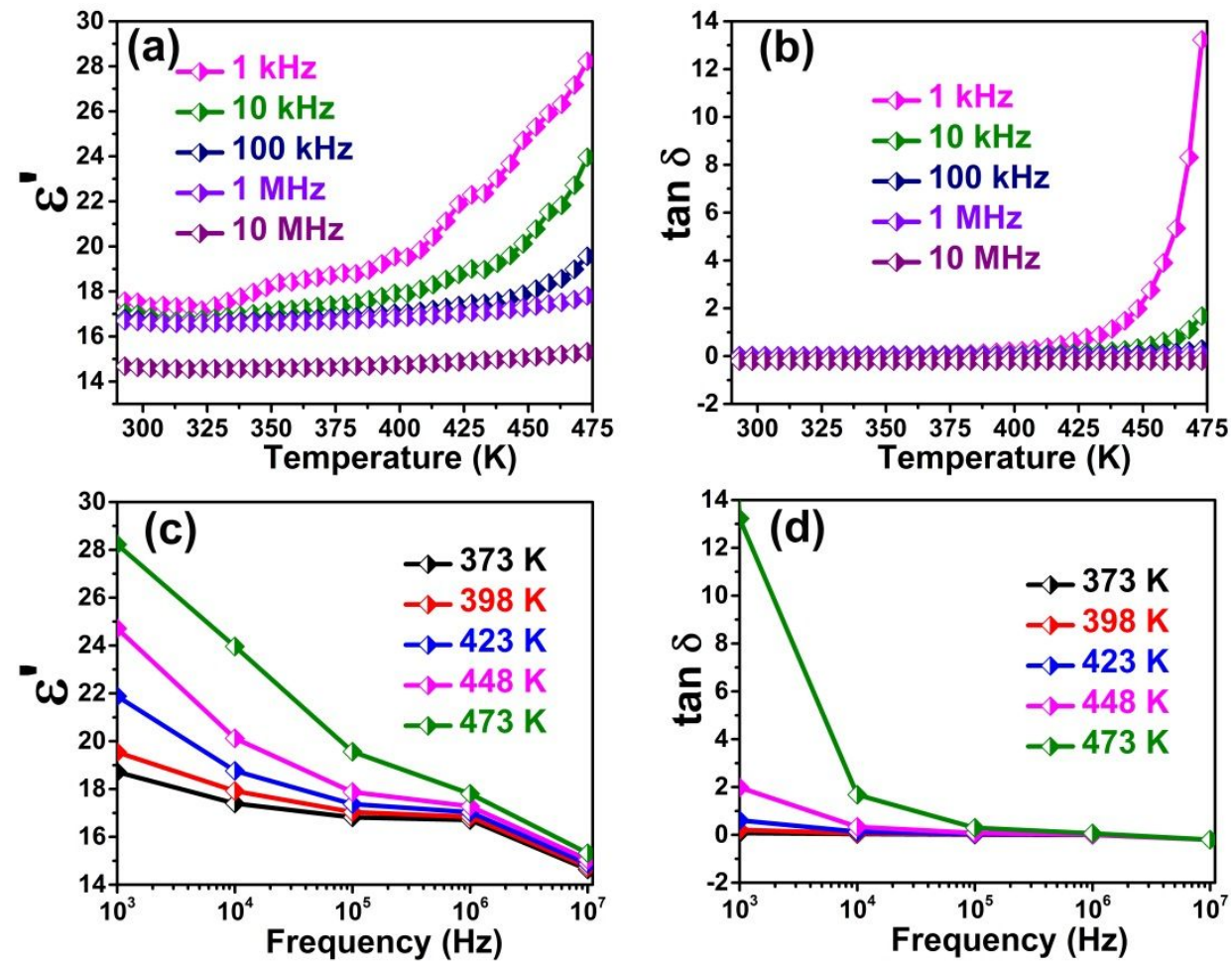

Figure S53. ( $a$ and b) temperature and (c and d) frequency dependent $\varepsilon^{\prime}$ and tan $\delta$ of TIAP·BF 4
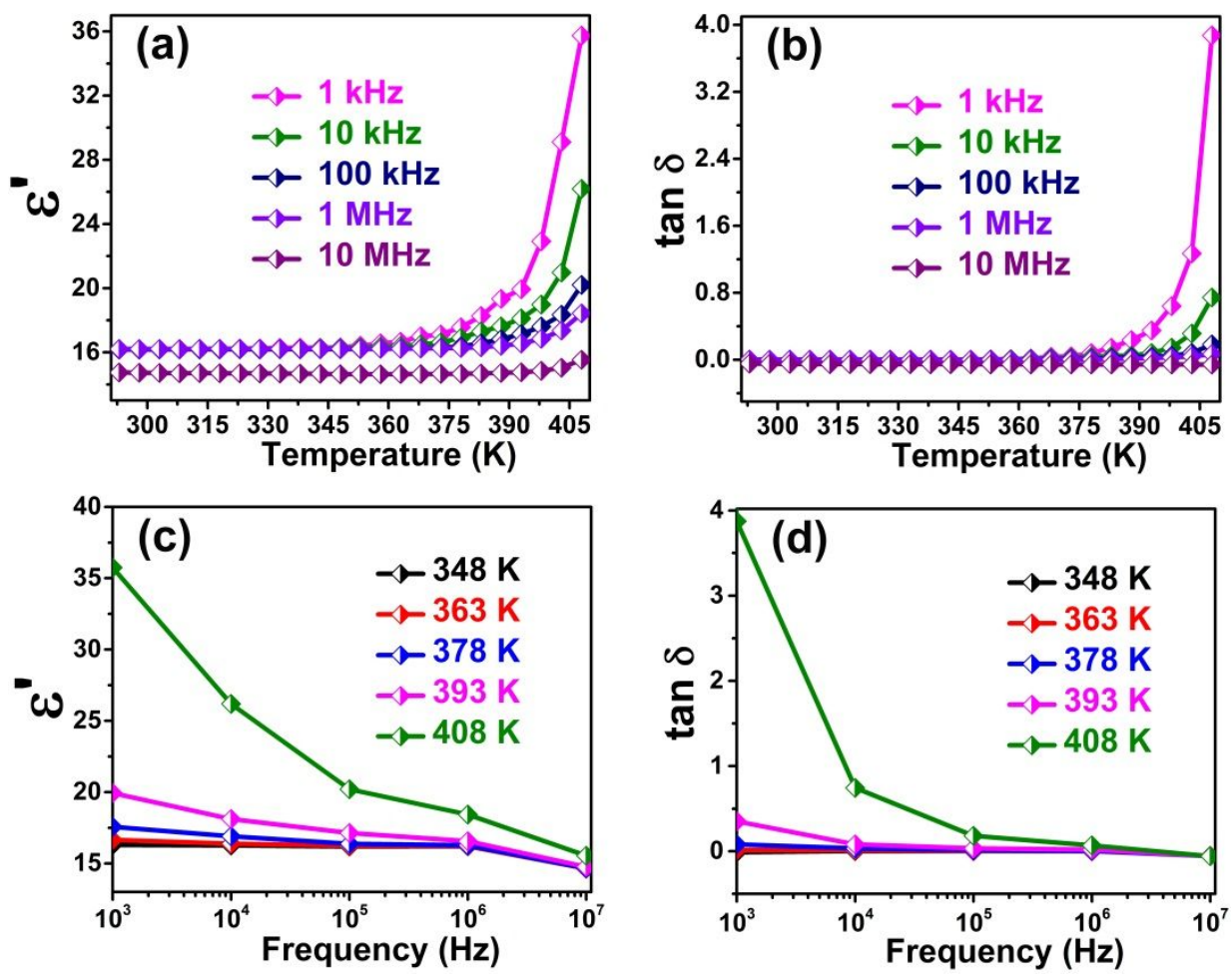

Figure S54. (a and b) temperature and (c and d) frequency dependent $\varepsilon^{\prime}$ and tan $\delta$ of TPAP.ClO 

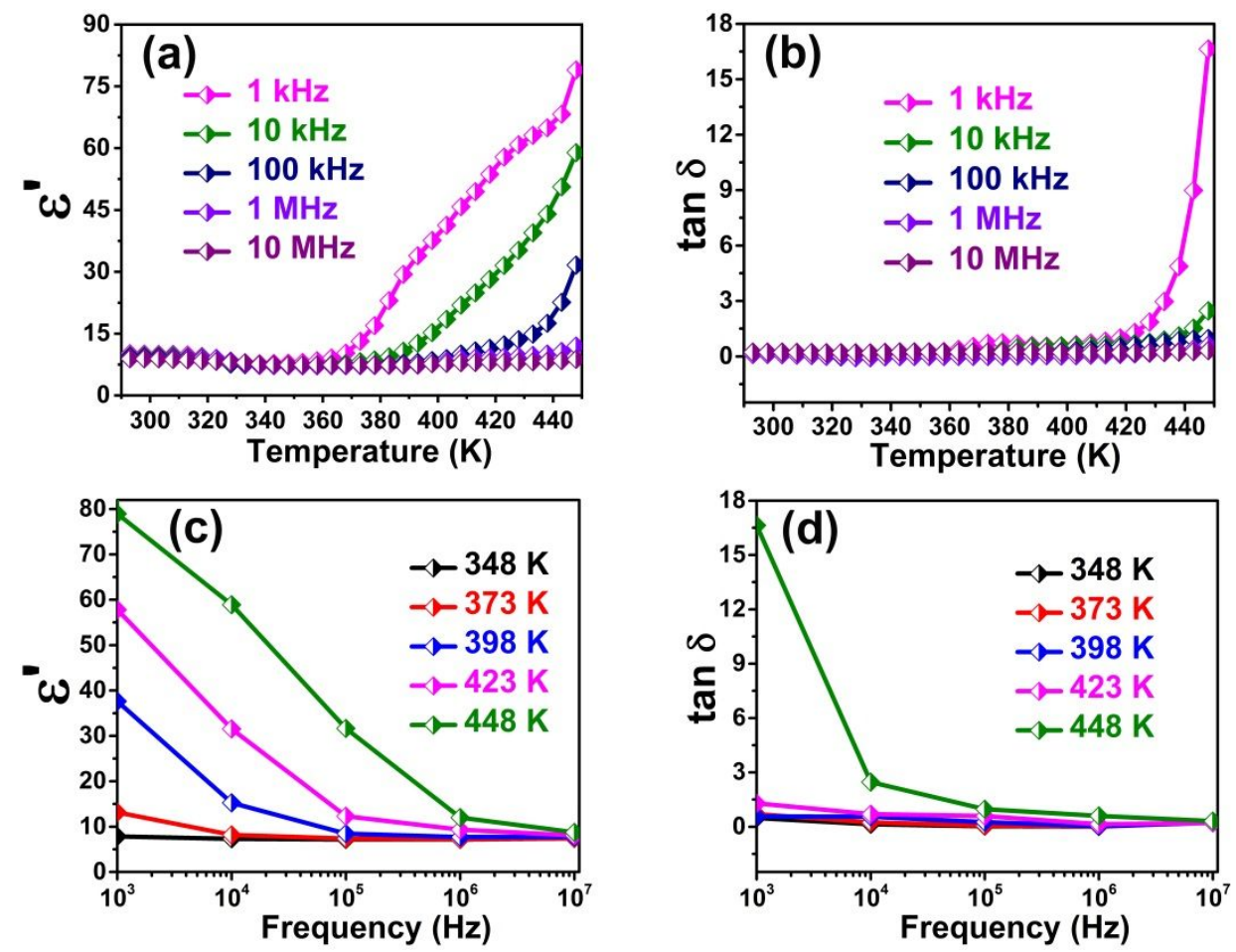

Figure S55. (a and b) temperature and (c and d) frequency dependent $\varepsilon^{\prime}$ and tan $\delta$ of DPDP. $\mathrm{ClO}_{4}$
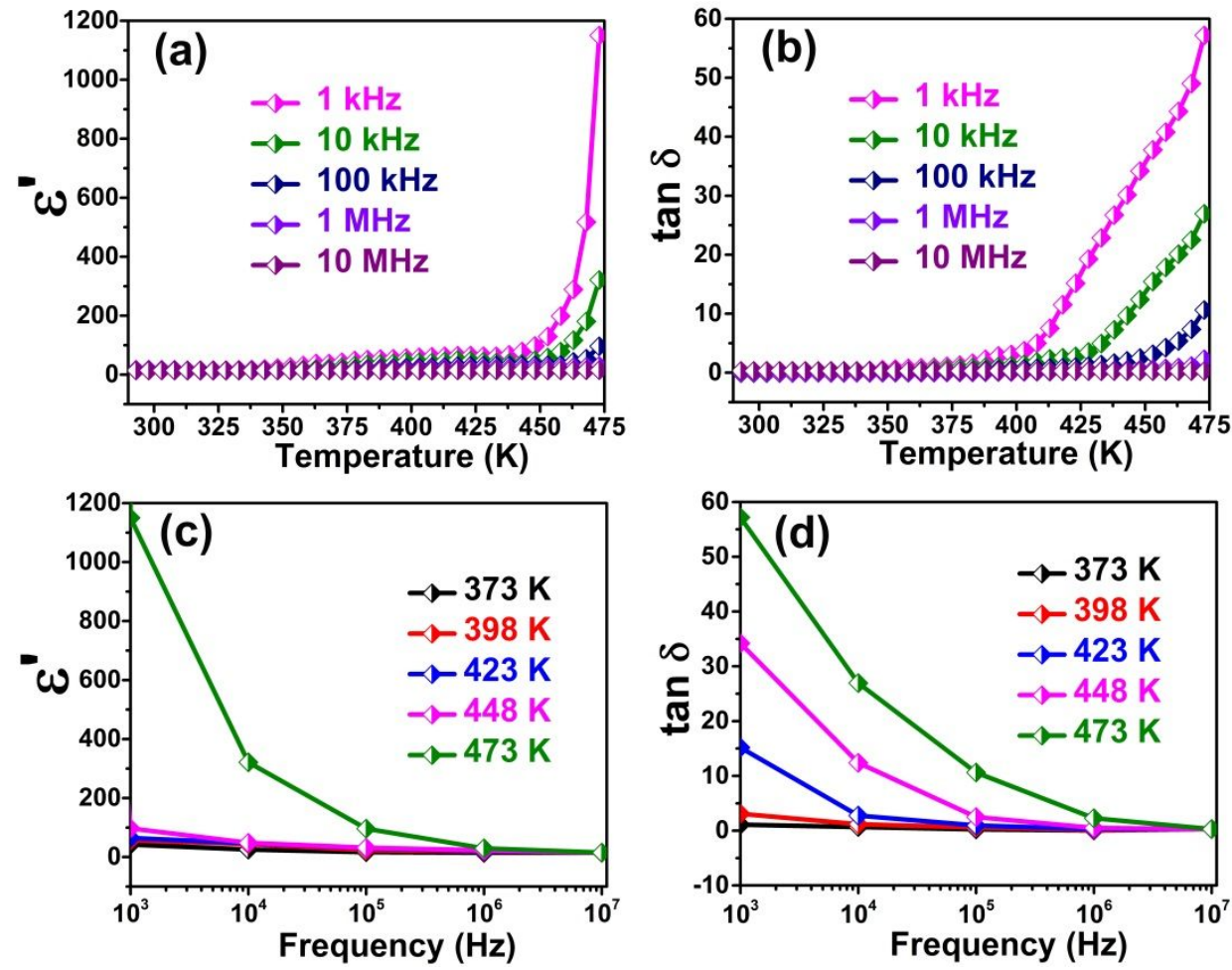

Figure S56. (a and b) temperature and (c and d) frequency dependent $\varepsilon^{\prime}$ and $\tan \delta$ of TIAP. $\mathrm{ClO}_{4}$ 

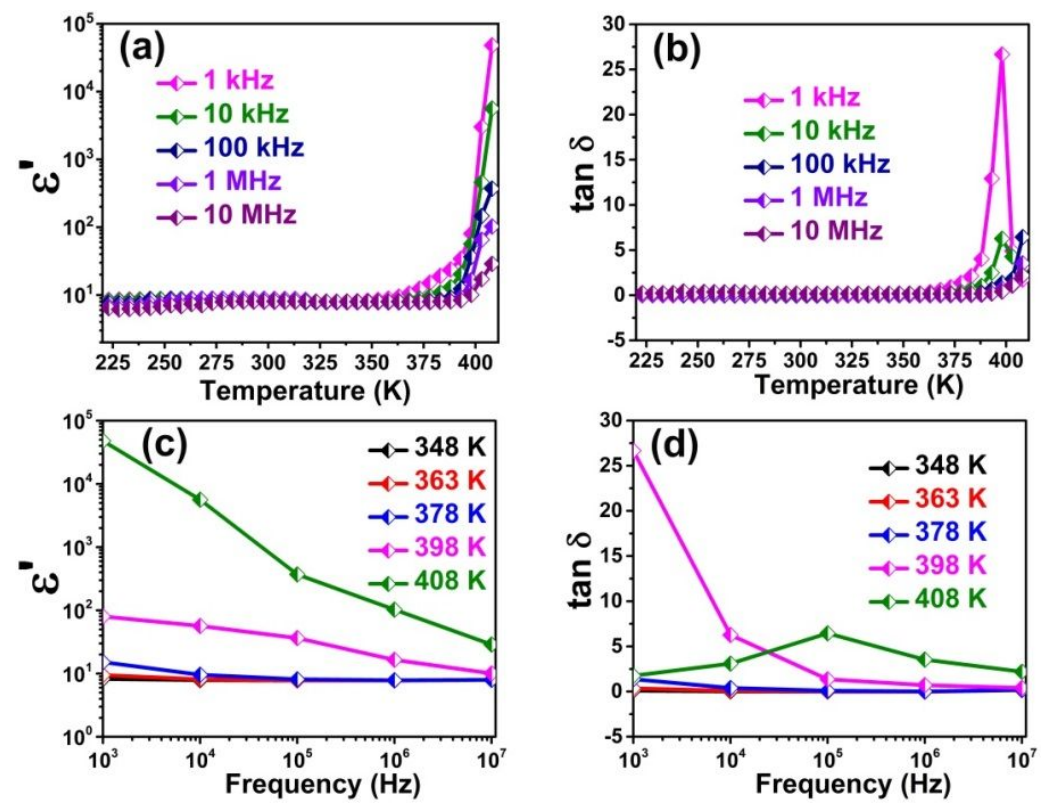

Figure S57. ( $a$ and $b$ ) temperature and (c and d) frequency dependent $\varepsilon^{\prime}$ and $\tan \delta$ of DPDP.IO

Table S7. Dipole moment calculation of all the ferroelectric phosphonium salts

\begin{tabular}{|l|l|l|}
\hline S.No & $\begin{array}{l}\text { Ferroelectric } \\
\text { Material }\end{array}$ & $\begin{array}{l}\text { Dipole moment } \\
\text { in Debye unit (D) }\end{array}$ \\
\hline 1 & TPAP·BF $_{4}$ & 18.5745 \\
\hline 2 & DPDP·BF $_{4}$ & 23.4453 \\
\hline 3 & DPDP·CIO $_{4}$ & 22.6718 \\
\hline 4 & DPDP·IO $_{4}$ & 21.9416 \\
\hline
\end{tabular}

Table S8. Piezoelectric coefficients $\left(d_{33}\right)$ of some of the well-known piezo and ferroelectric materials

\begin{tabular}{|c|c|c|c|}
\hline S.No & $\begin{array}{c}\text { Ferro and Piezoelectric } \\
\text { Materials }\end{array}$ & $d_{33}(p C / N)$ & Reference \\
\hline 1 & Imidazolium periodate & 4.2 & 2 \\
\hline 2 & Croconic acid & 5 & 5 \\
\hline 3 & Benzil & 6 & 3 \\
\hline 4 & Rochelle salt & 7 & 5 \\
\hline 5 & HPLN & 7.3 & 3 \\
\hline 6 & Guanidinium perchlorate & 10 & 4 \\
\hline 7 & PhMDA & 10.4 & 3 \\
\hline 8 & DIPAB & 11 & 5 \\
\hline 9 & $\mathrm{LiNbO}_{3}$ & 8 & 4 \\
\hline 10 & $\begin{array}{c}\text { (N-methyl } \\
\text { pyrrolidinium })_{3} \mathrm{Sb}_{2} \mathrm{Br}_{9}\end{array}$ & 12 & 6 \\
\hline 11 & $\begin{array}{l}\text { Trimethylchloromethyl } \\
\text { ammonium } \\
\text { trichloromanganese(II) }\end{array}$ & 185 & 5 \\
\hline 12 & TPAP $\cdot \mathrm{BF}_{4}$ & 3 & This work \\
\hline 13 & $\mathrm{DPDP} \cdot \mathrm{BF}_{4}$ & 7 & This work \\
\hline 14 & TIAP·BF 4 & 3 & This work \\
\hline 15 & TPAP $\cdot \mathrm{ClO}_{4}$ & 4 & This work \\
\hline 16 & DPDP. $\mathrm{ClO}_{4}$ & 12 & This work \\
\hline 17 & $\mathrm{TIAP} \cdot \mathrm{ClO}_{4}$ & 3 & This work \\
\hline 18 & $\mathrm{DPDP} \cdot \mathrm{IO}_{4}$ & 8 & This work \\
\hline
\end{tabular}


Table S9. Details about the preparation of various weight percentage (wt\%) composites of the organo-amino phosphonium salts

\begin{tabular}{|c|c|c|c|}
\hline $\begin{array}{l}\text { Organic polymer } \\
\text { composites Name }\end{array}$ & $\begin{array}{c}\text { Composite } \\
\text { (wt\%) }\end{array}$ & $\begin{array}{l}\text { Salt amount } \\
\quad \text { (in } \mathrm{mg} \text { ) }\end{array}$ & $\begin{array}{c}\text { TPU + Organic } \\
\text { salts (in mg) }\end{array}$ \\
\hline $\mathrm{TPAP} \cdot \mathrm{BF}_{4} / \mathrm{TPU}$ & $\begin{array}{c}5 \\
10 \\
15 \\
20\end{array}$ & $\begin{array}{c}53 \\
112 \\
177 \\
250\end{array}$ & $\begin{array}{l}1053 \\
1112 \\
1177 \\
1250\end{array}$ \\
\hline $\mathrm{DPDP} \cdot \mathrm{BF}_{4} / \mathrm{TPU}$ & $\begin{array}{c}5 \\
10 \\
15 \\
20\end{array}$ & $\begin{array}{c}53 \\
112 \\
177 \\
250\end{array}$ & $\begin{array}{l}1053 \\
1112 \\
1177 \\
1250\end{array}$ \\
\hline $\mathrm{TIAP} \cdot \mathrm{BF}_{4} / \mathrm{TPU}$ & $\begin{array}{c}5 \\
10 \\
15 \\
20\end{array}$ & $\begin{array}{c}53 \\
112 \\
177 \\
250\end{array}$ & $\begin{array}{l}1053 \\
1112 \\
1177 \\
1250\end{array}$ \\
\hline DPDP.PF $/$ /TPU & $\begin{array}{c}5 \\
10 \\
15 \\
20\end{array}$ & $\begin{array}{c}53 \\
112 \\
177 \\
250\end{array}$ & $\begin{array}{l}1053 \\
1112 \\
1177 \\
1250\end{array}$ \\
\hline
\end{tabular}
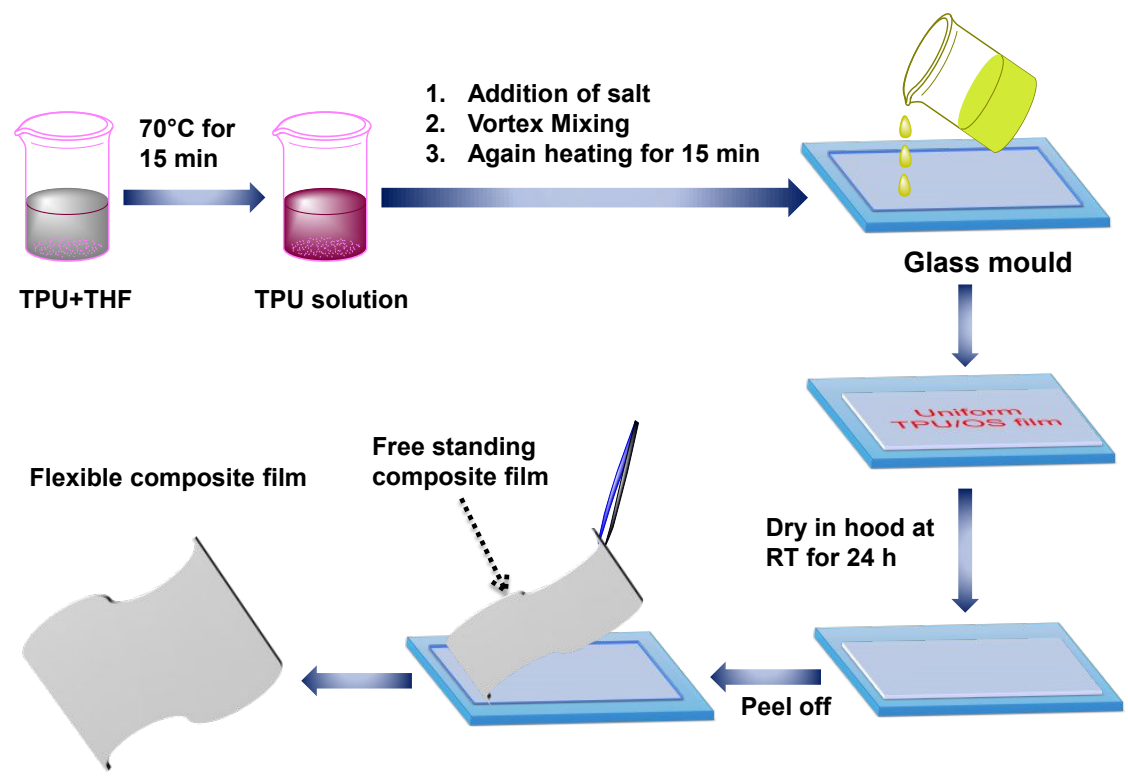

Scheme S5. Detailed procedure involved for making various wt $\%(5,10,15$ and 20) polymeric composite films 


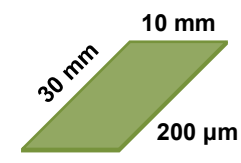

copper electrode

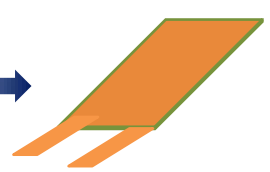

Lead attachment

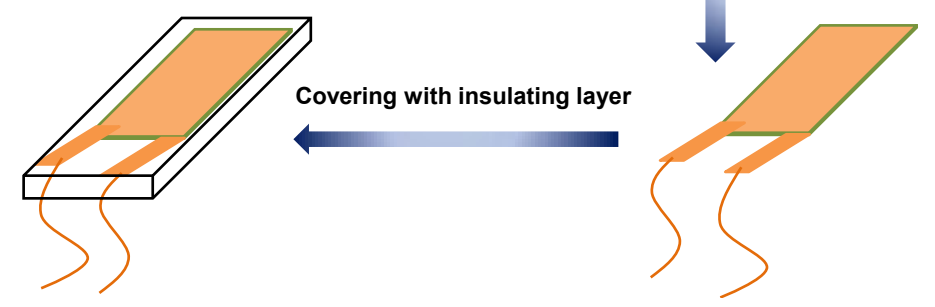

Scheme S6. Device fabrication of all the wt $\%$ of $(5,10,15$ and 20$)$ composite films

(a)

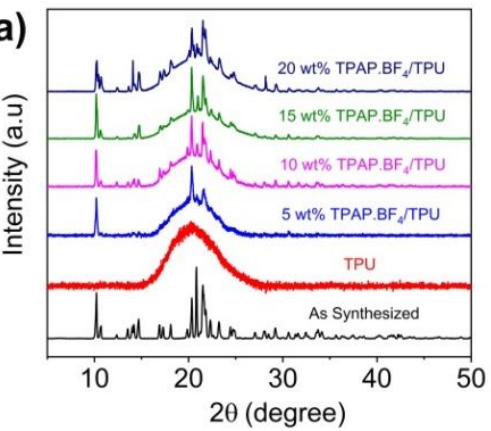

(b)

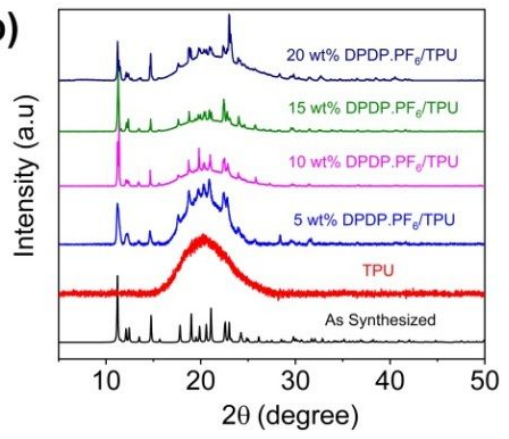

Figure S58. PXRD analysis of all wt $\%$ composite films of $\mathrm{TPAP}^{\mathrm{B}} \mathrm{BF}_{4} / \mathrm{TPU}$ and DPDP.PF 6 /TPU

(a)

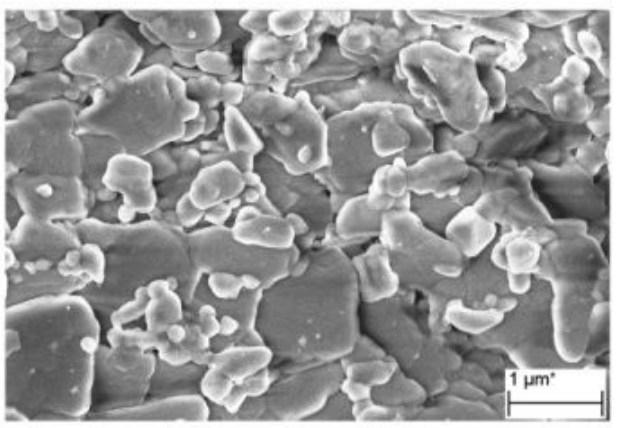

(c)

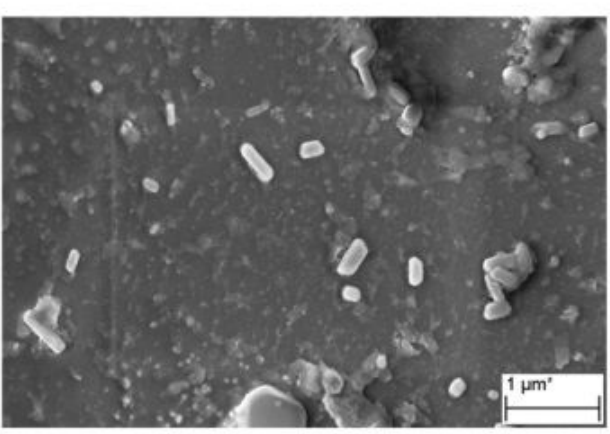

(b)

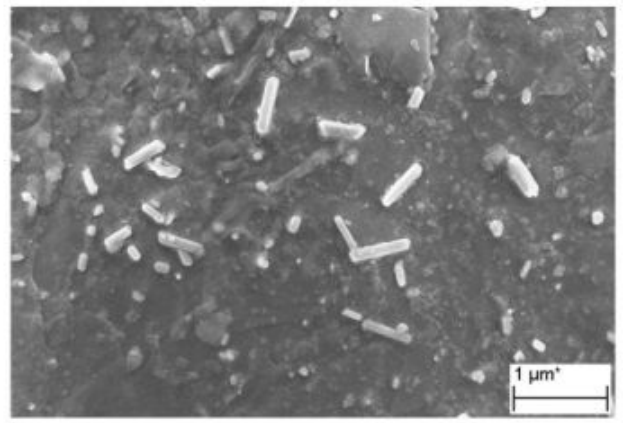

(d)

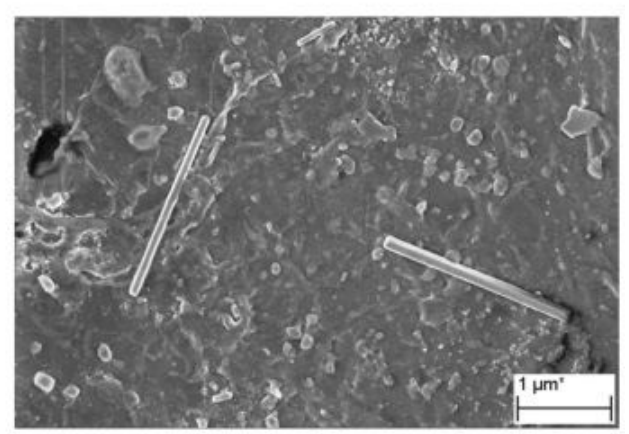

Figure S59. SEM images of the (a) 5 (b) 10 (c) 15 and (d) 20 wt\% TPAP.BF $/$ TPU films 
(a)

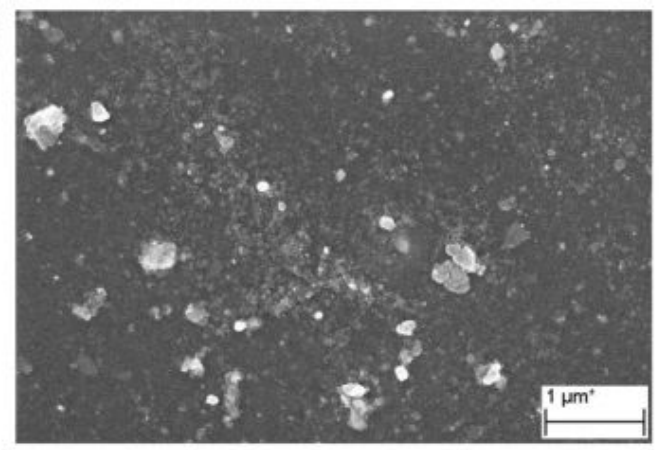

(c)

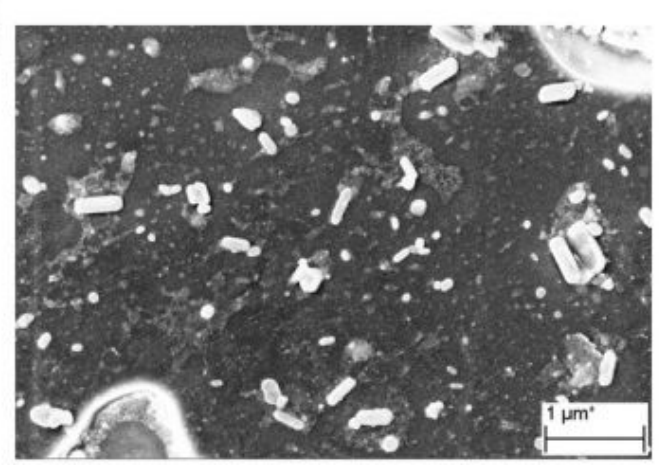

(b)

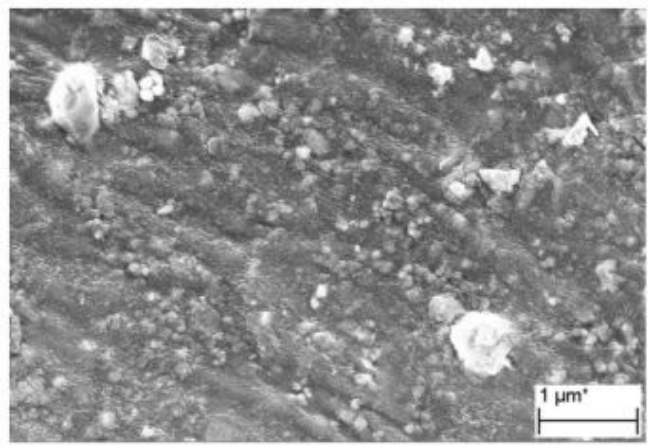

(d)

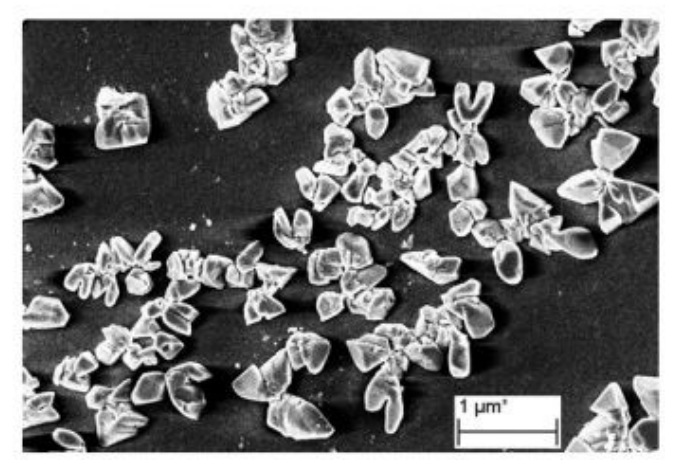

Figure S60. SEM images of (a) 5 (b) 10 (c) 15 and (d) 20 wt\% DPDP.BF ${ }_{4} /$ TPU films

(a)

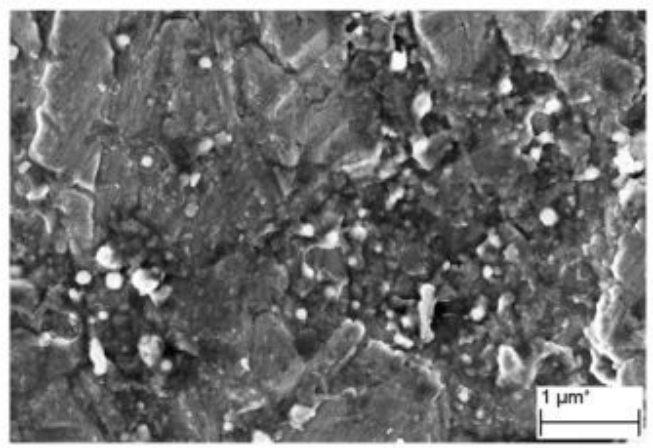

(c)

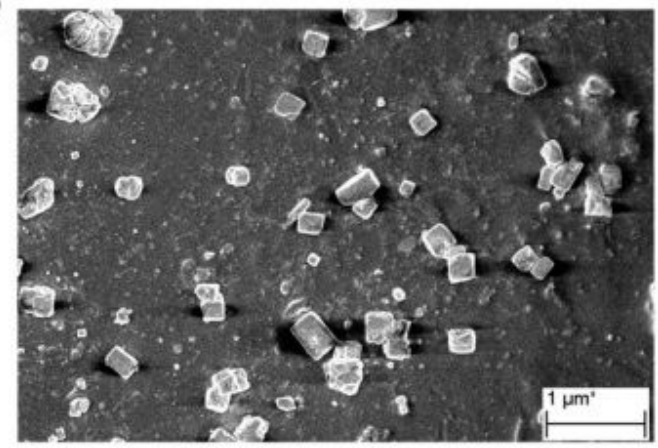

(b)

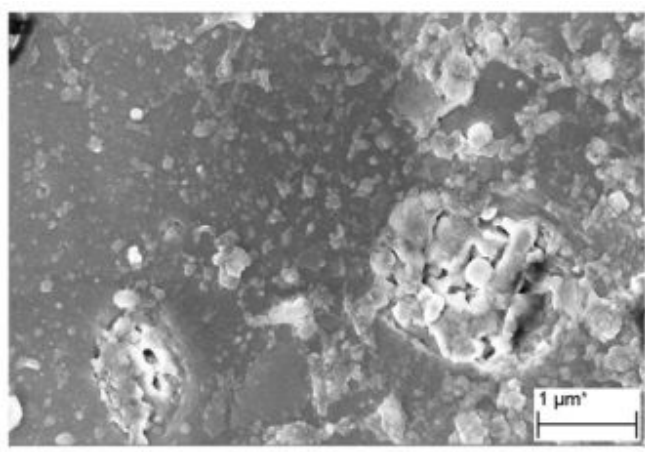

(d)

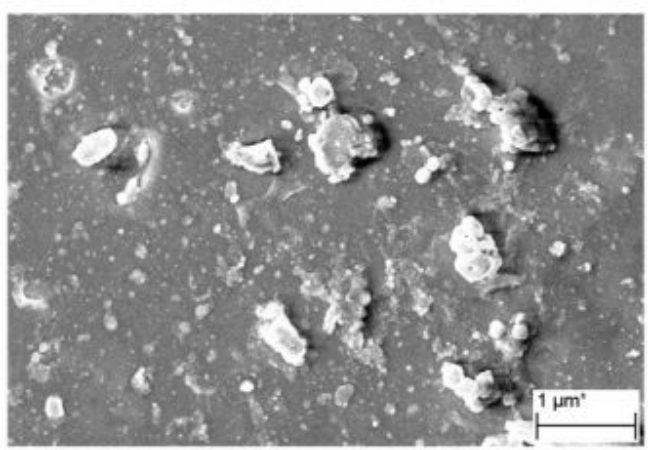

Figure S61. SEM images of (a) 5 (b) 10 (c) 15 and (d) $20 \mathrm{wt} \% \mathrm{TIAP} \cdot \mathrm{BF}_{4} / \mathrm{TPU}$ films 
(a)

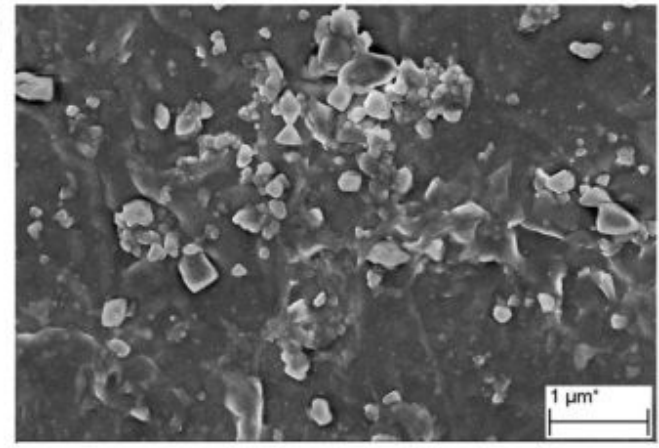

(c)

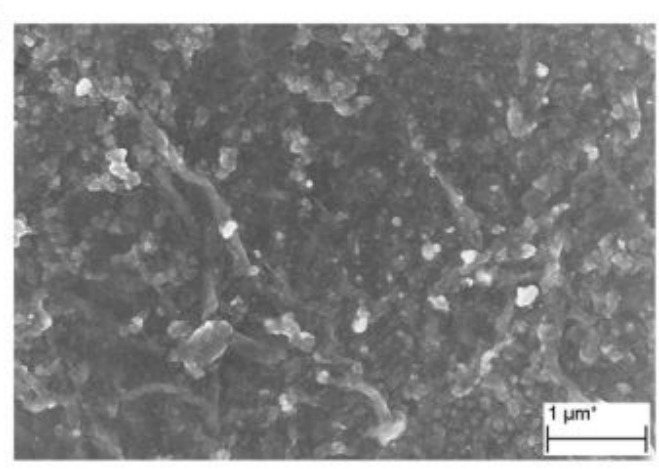

(b)

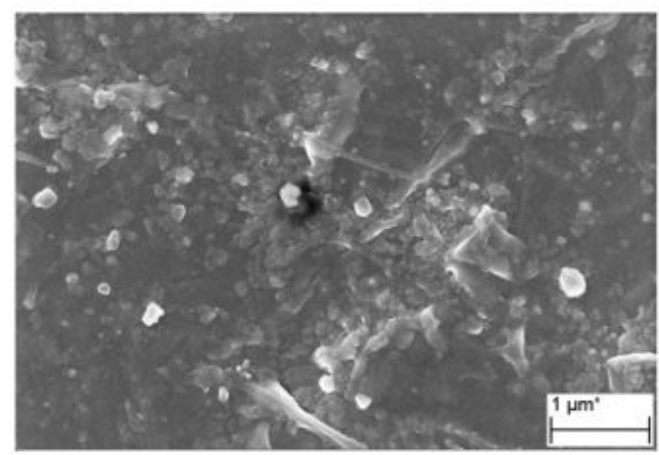

(d)

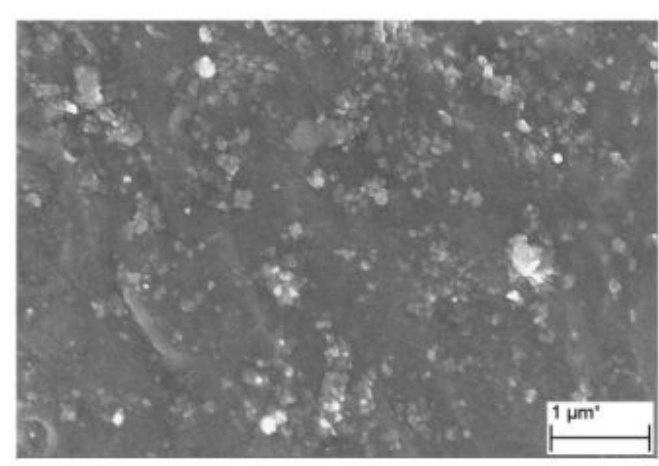

Figure S62. SEM images of (a) 5 (b) 10 (c) 15 and (d) $20 \mathrm{wt} \%$ DPDP.PF $6 /$ TPU films
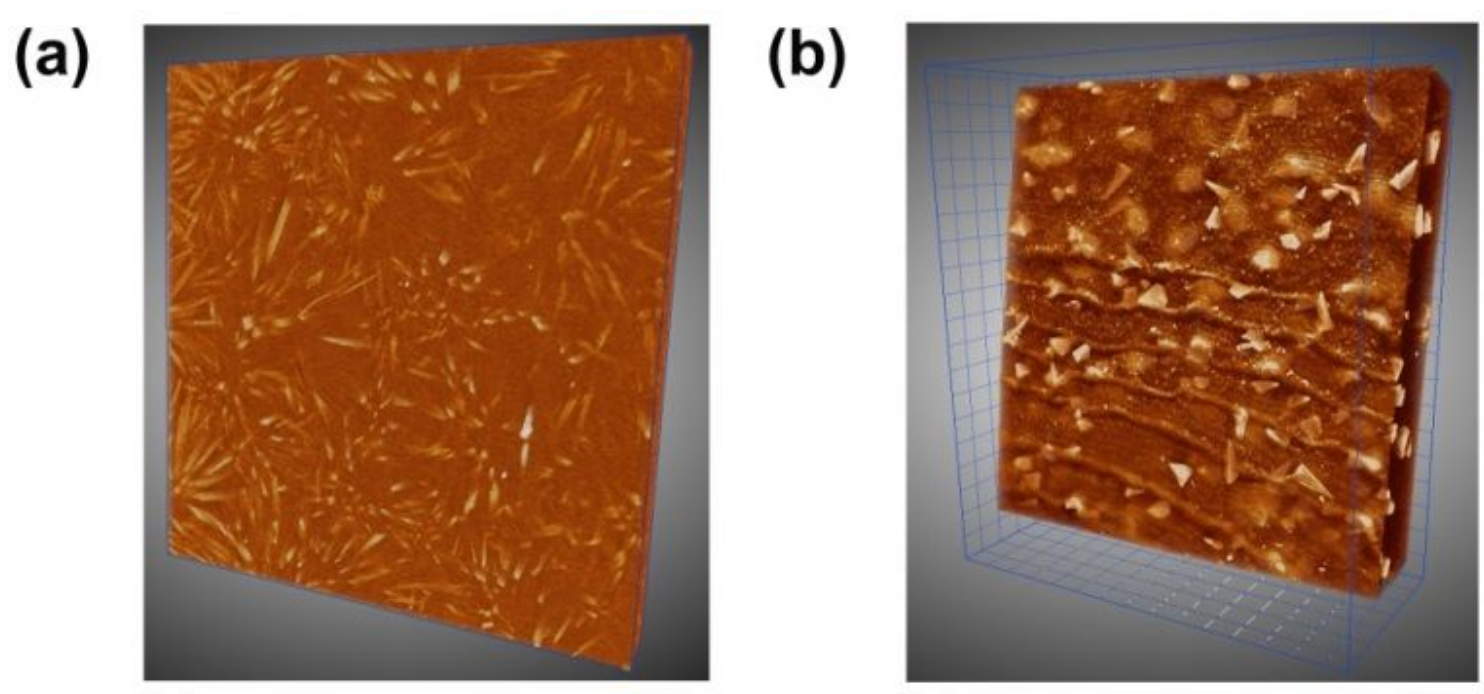

Figure S63. X-ray 3D-tomography analysis of (a) TPAP·BF $/$ TPU and (b) DPDP.PF $/$ TPU. The tomography images depicted here is for the corresponding $10 \mathrm{wt} \%$ composite films (grid-scale: $100 \mu \mathrm{m}$ ) 
(a)

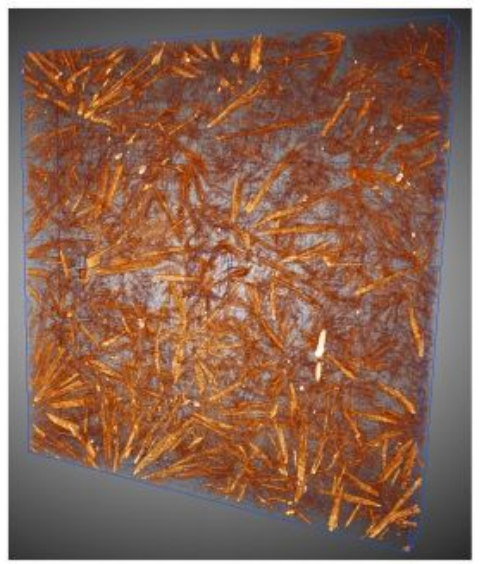

(c)

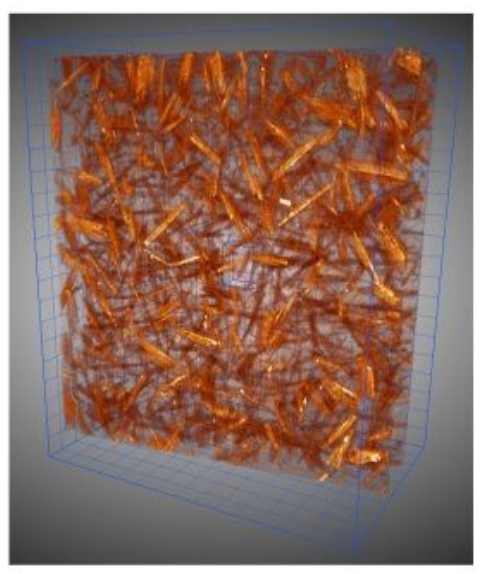

(b)

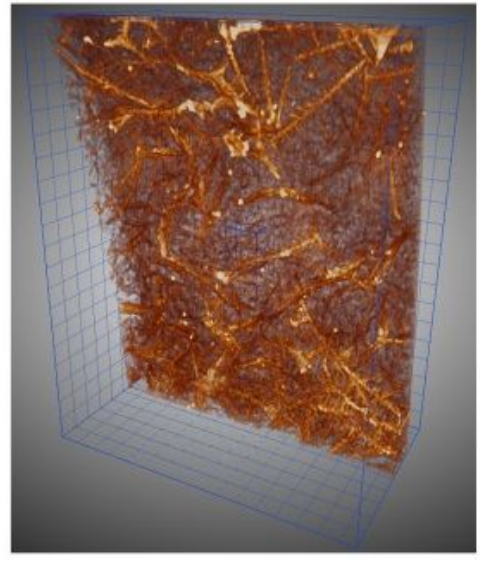

(d)

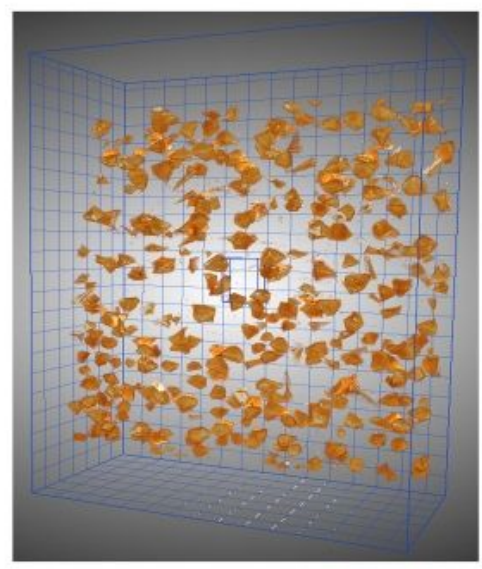

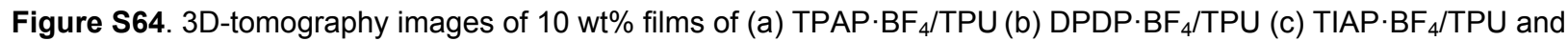
(d) DPDP.PF 6 /TPU. The polymer background has been corrected to indicate the distribution of the crystallites.

(a)

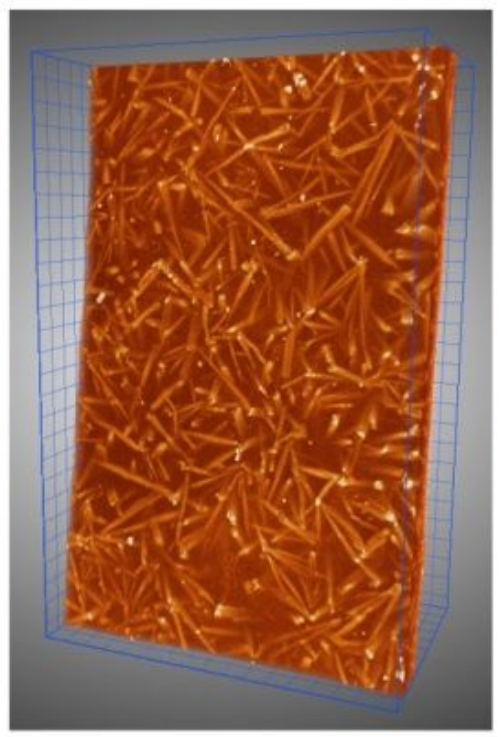

(b)

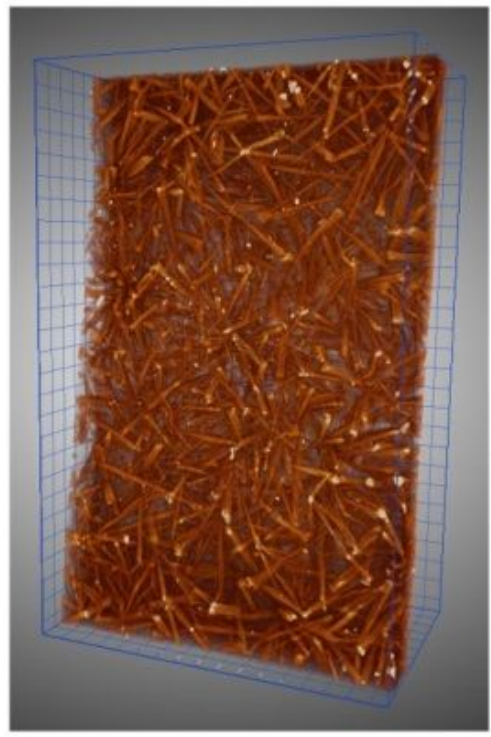

Figure S65. (a) 3D-tomography images of $20 \mathrm{wt} \% \mathrm{TPAP} \cdot \mathrm{BF}_{4} / \mathrm{TPU}$ composite film and (b) image obtained after the polymer background correction. 
(a)

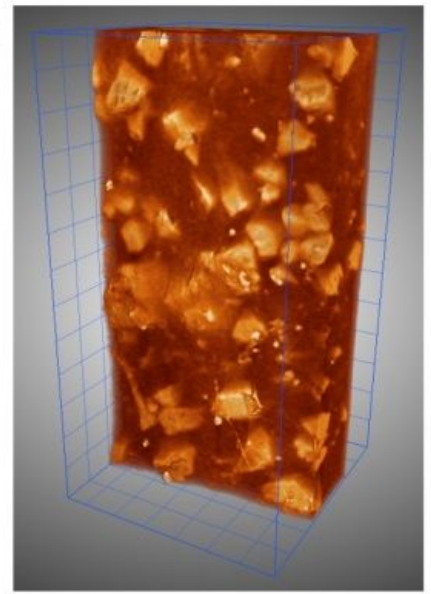

(b)

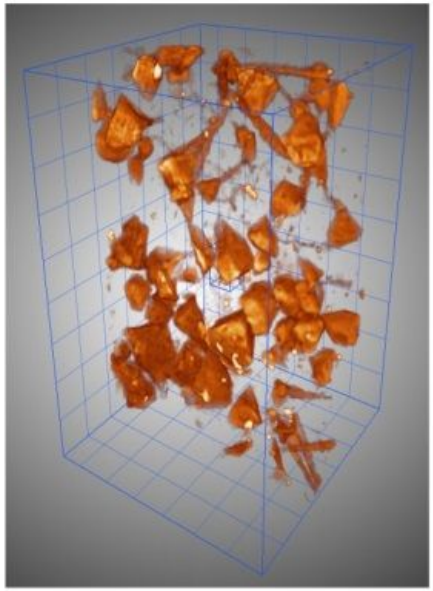

Figure S66. 3D-tomography images of $20 \mathrm{wt} \% \mathrm{DPDP} \cdot \mathrm{BF}_{4} / \mathrm{TPU}$ composite film and (b) image obtained after the polymer background correction.

(a)

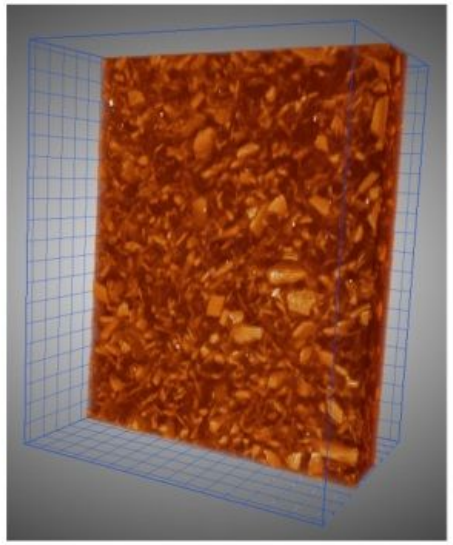

(b)

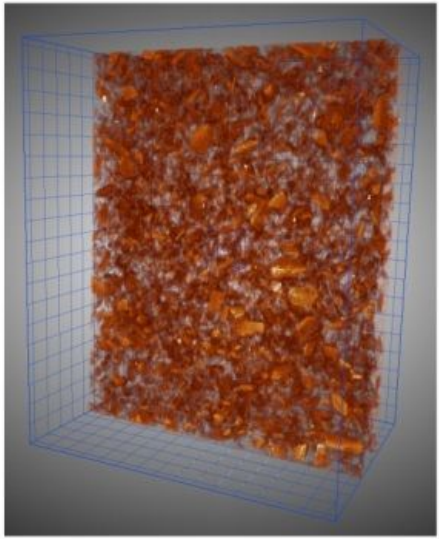

Figure S67. 3D-tomography images of $20 \mathrm{wt} \% \mathrm{TIAP} \cdot \mathrm{BF}_{4} / \mathrm{TPU}$ composite film and (b) image obtained after the polymer background correction.

(a)

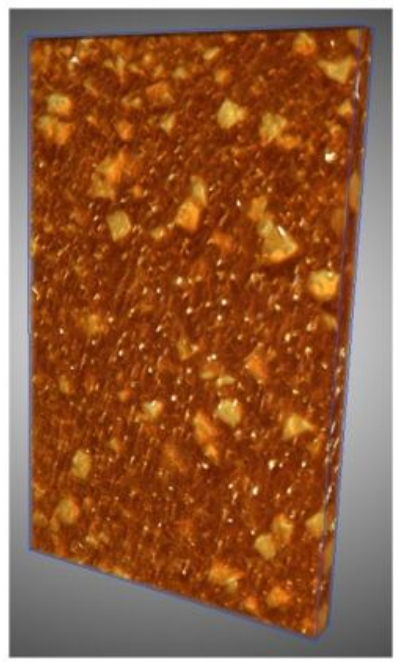

(b)

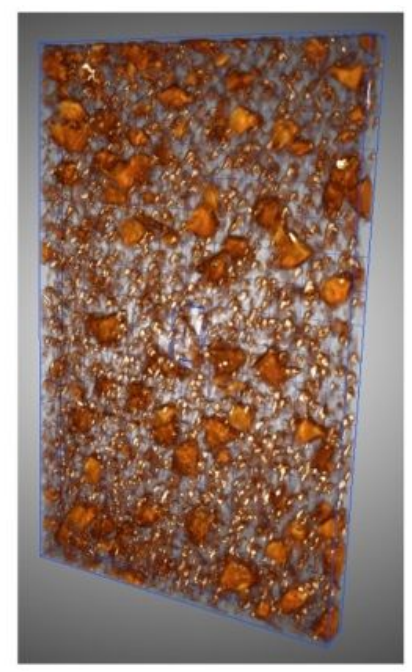

Figure S68. 3D-tomography images of $20 \mathrm{wt} \% \mathrm{DPDP} \cdot \mathrm{PF}_{6} / \mathrm{TPU}$ composite films and (b) image obtained after the polymer background correction. 

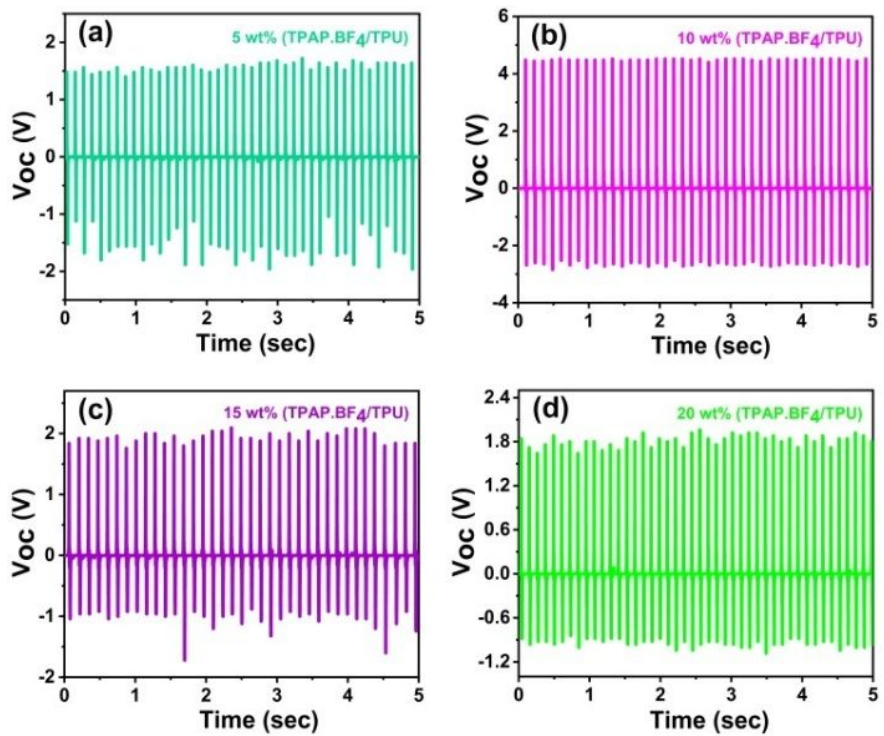

Figure S69. Voltage output performance for $\mathrm{TPAP}^{\mathrm{B} F}{ }_{4} / \mathrm{TPU}$ composites $(5,10,15$ and $20 \mathrm{wt} \%)$
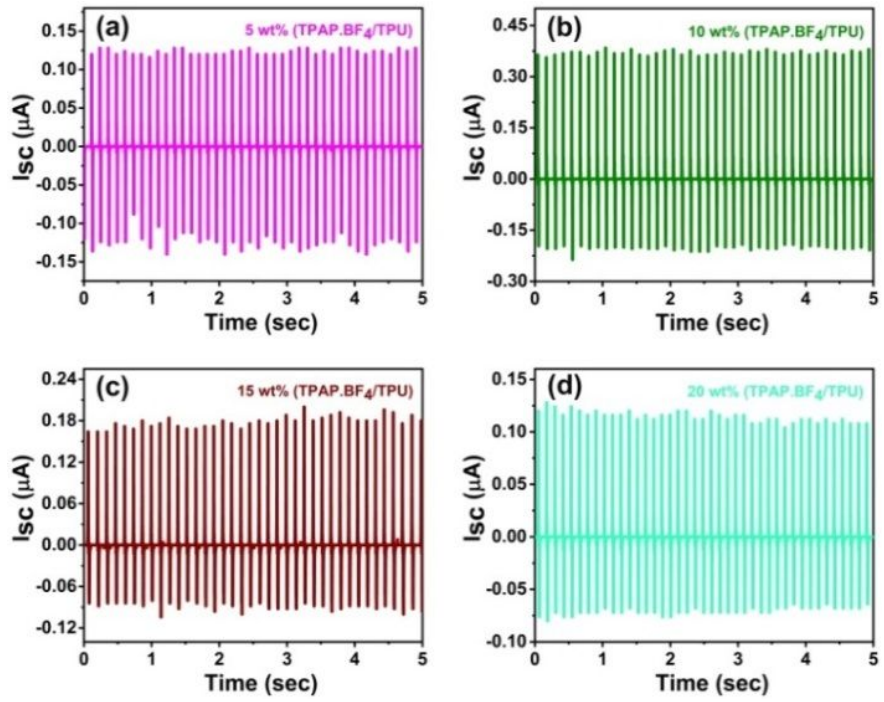

Figure S70. Current output performance for TPAP.BF 4 /TPU composites $(5,10,15$ and $20 \mathrm{wt} \%)$

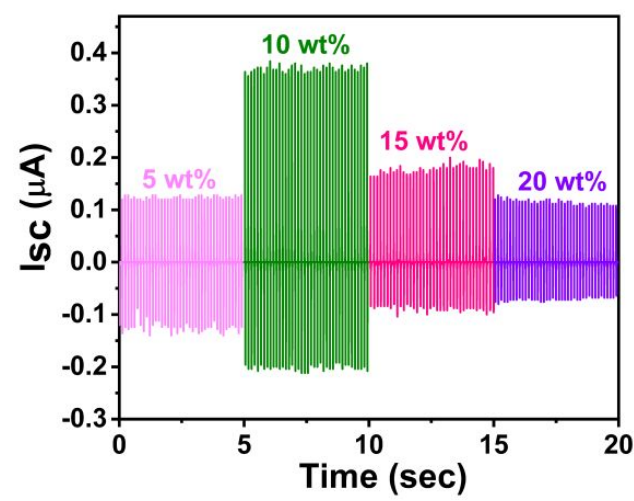

Figure S71. Comparison of the output current performances for the TPAP. $\mathrm{BF}_{4} / \mathrm{TPU}$ in all wt $\%$ composite films 


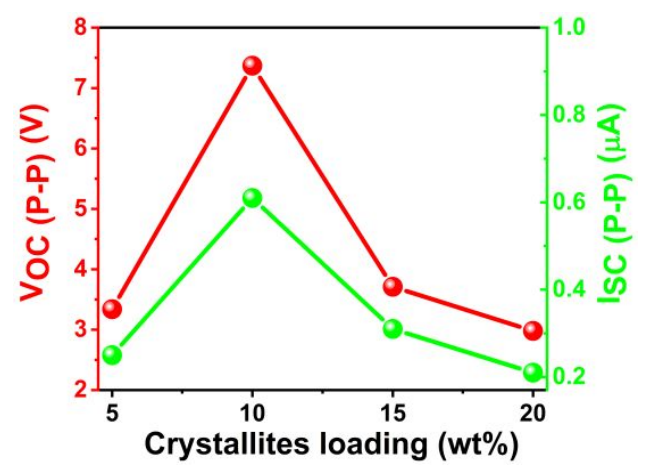

Figure S72. Output voltages and output currents of TPAP·BF $4 / T P U$ in all $w t \%$ composite films
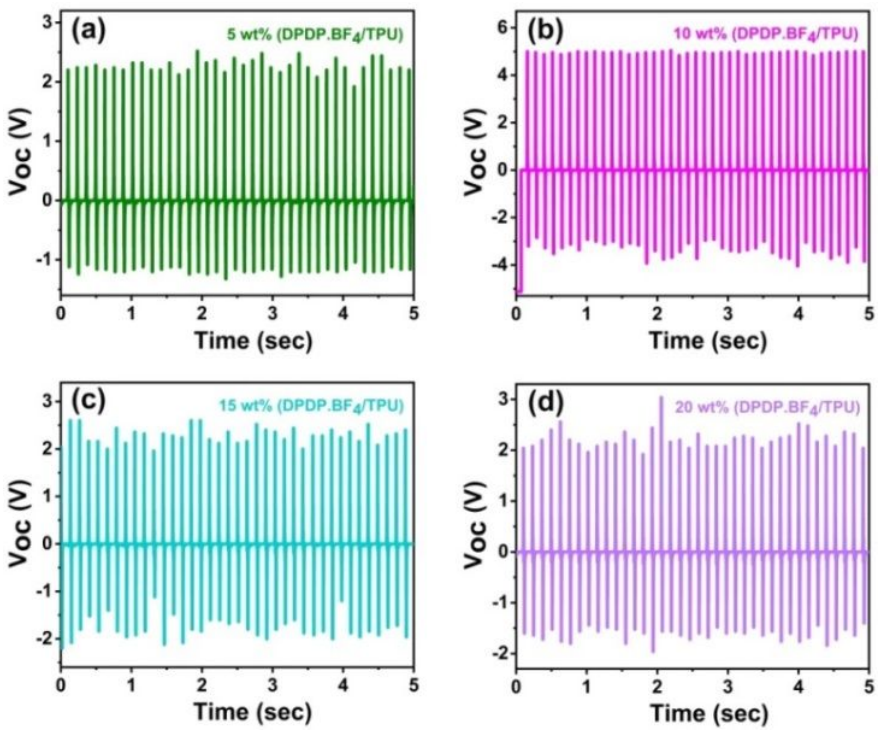

Figure S73. Voltage output performance for DPDP.BF ${ }_{4} / \mathrm{TPU}$ composites $(5,10,15$ and $20 \mathrm{wt} \%)$
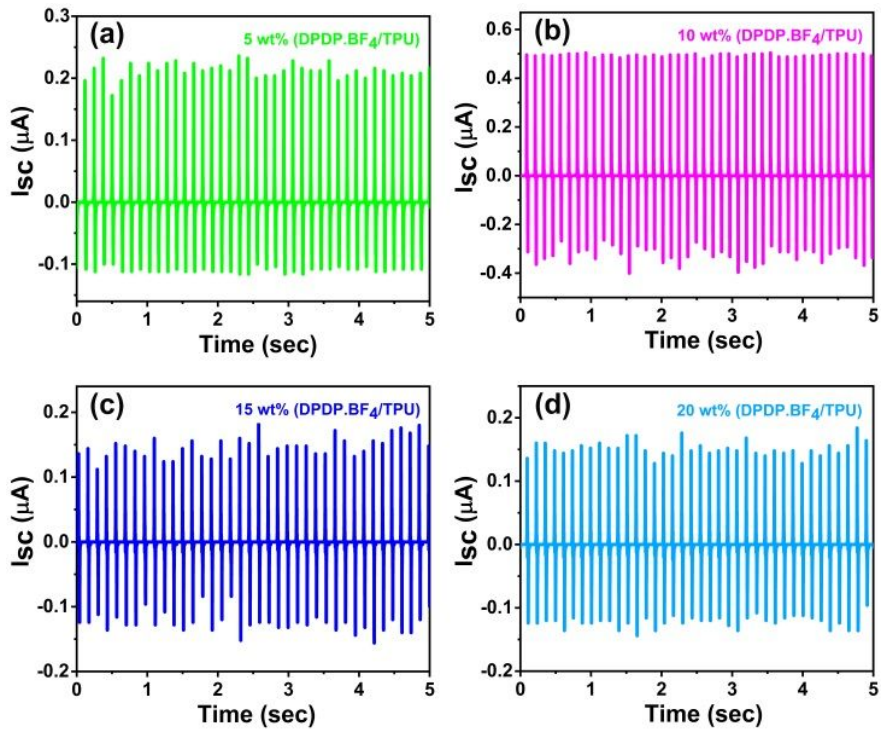

Figure S74. Current output performance for DPDP·BF $/$ TPU composites $(5,10,15$ and $20 \mathrm{wt} \%)$ 


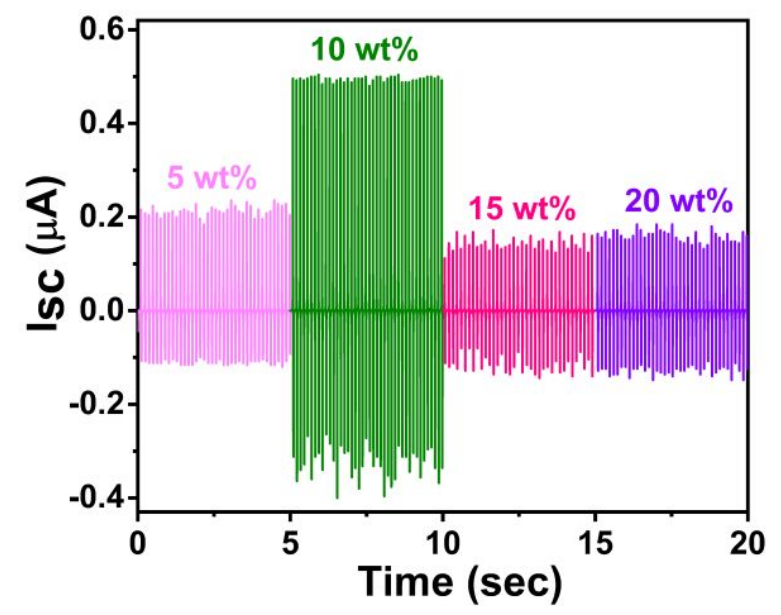

Figure S75. Comparison of output current performance for DPDP.BF $4 / T P U$ in all wt $\%$ composite films

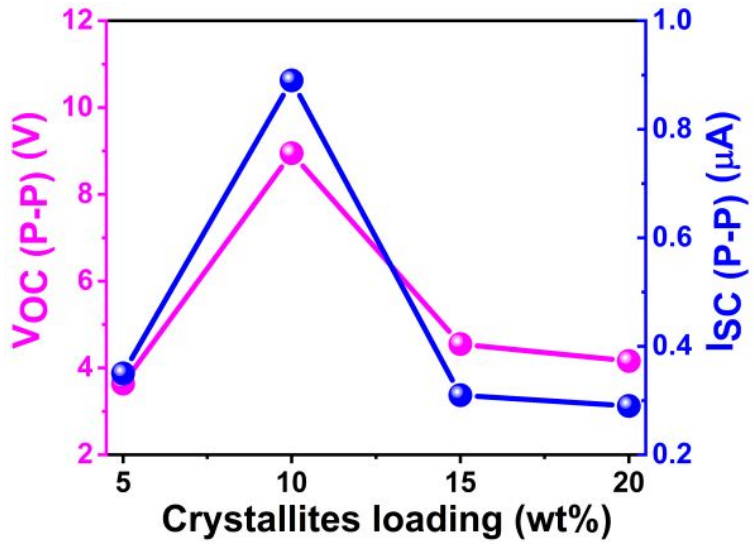

Figure S76. Output voltages and output currents of DPDP.BF $/$ TPU composite films in all wt $\%$ composite films
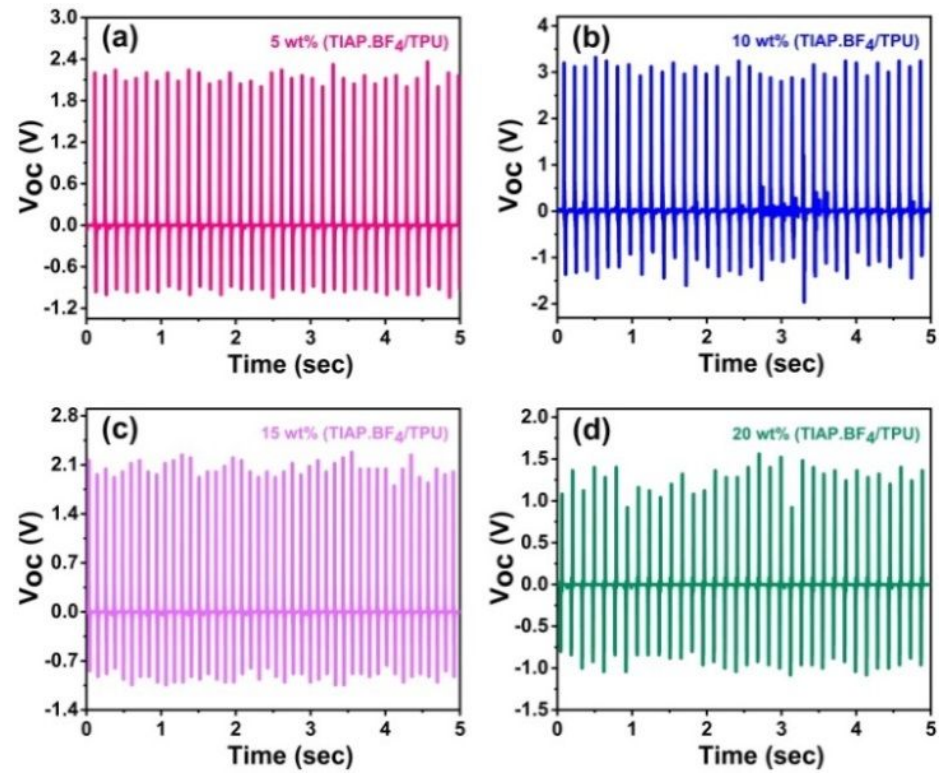

Figure S77. Voltage output performance for TIAP·BF 4 TPU composite films in all wt\% composite films 

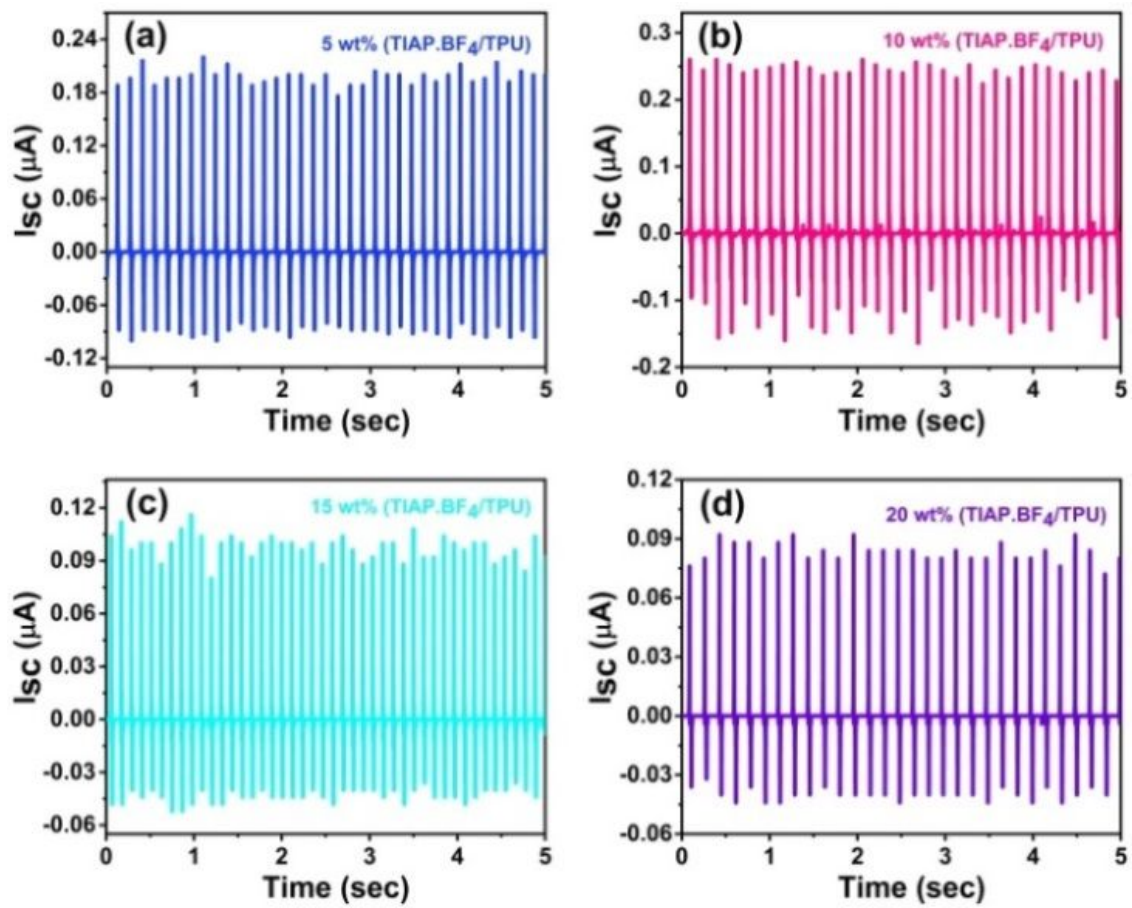

Figure S78. Current output performance for TIAP·BF$/ / T P U$ composite films in all wt $\%$ composite films

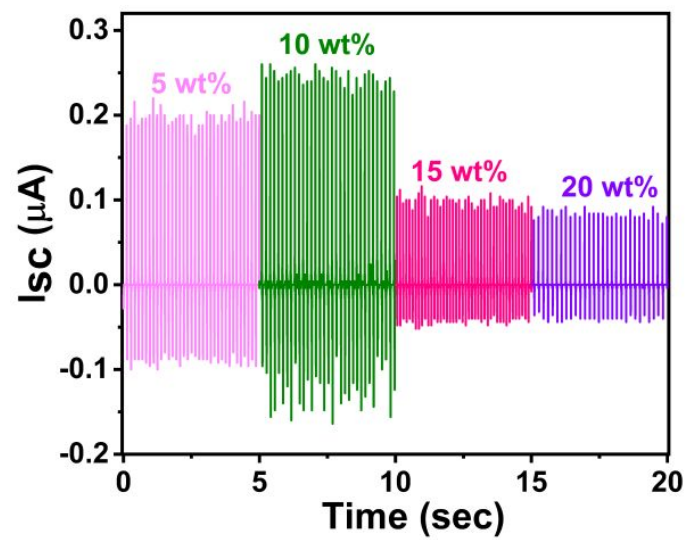

Figure S79. Comparison of output current performance for TIAP.BF $4 / T P U$ composite films in all wt $\%$ composite films

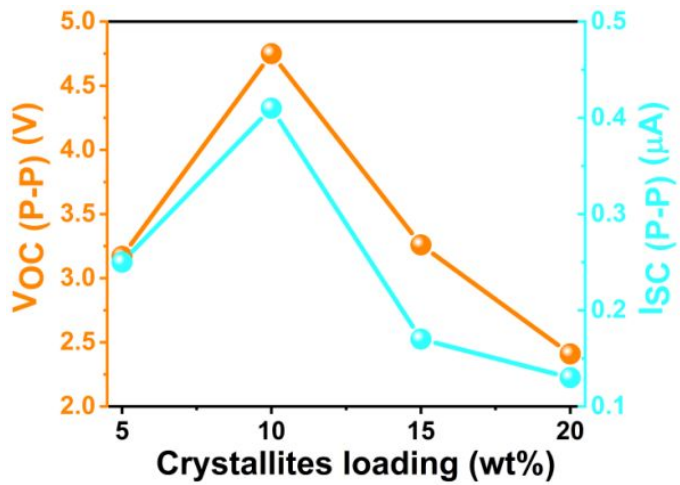

Figure S80. Output voltages and output currents of TIAP·BF $/ \mathrm{TPU}$ composite films in all $\mathrm{wt} \%$ composite films 

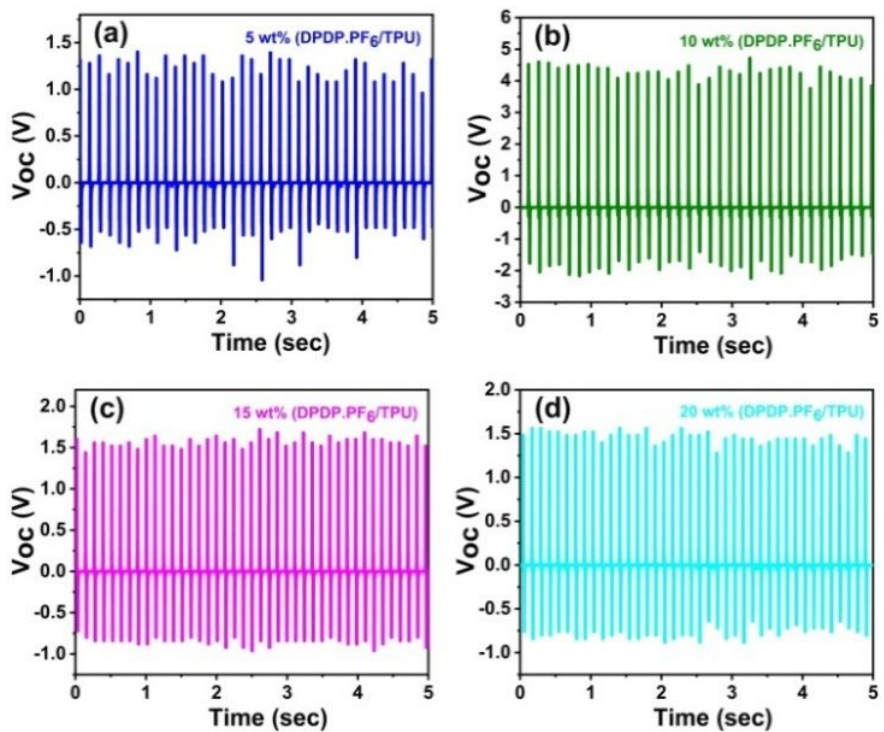

Figure S81. Voltage output performance for DPDP.PF 6 /TPU composite films in all wt $\%$ composite films
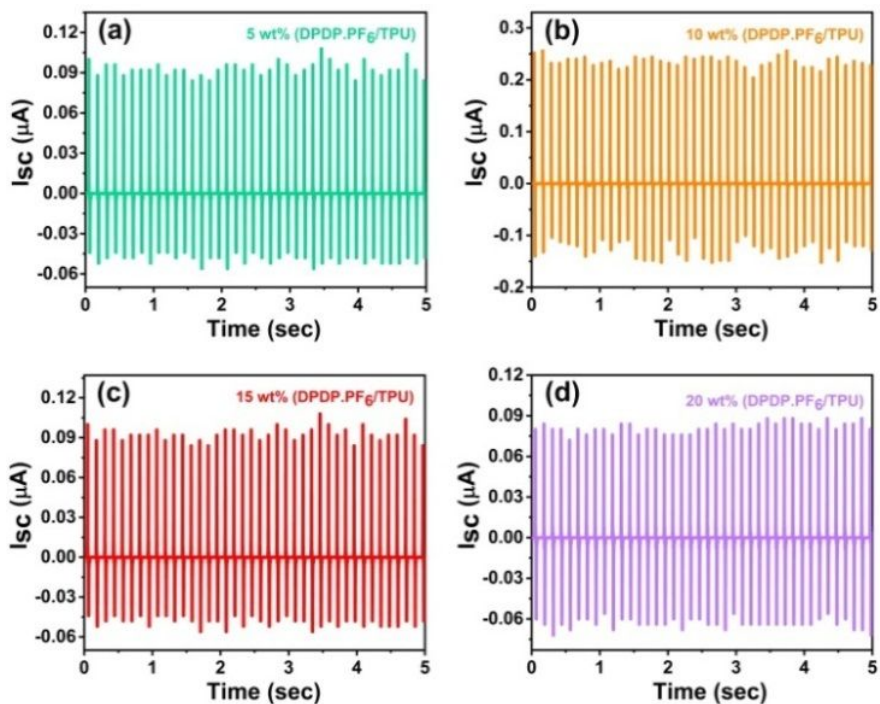

Figure S82. Current output performance for DPDP.PF $/ \mathrm{TPU}$ composite films in all $\mathrm{wt} \%$ composite films

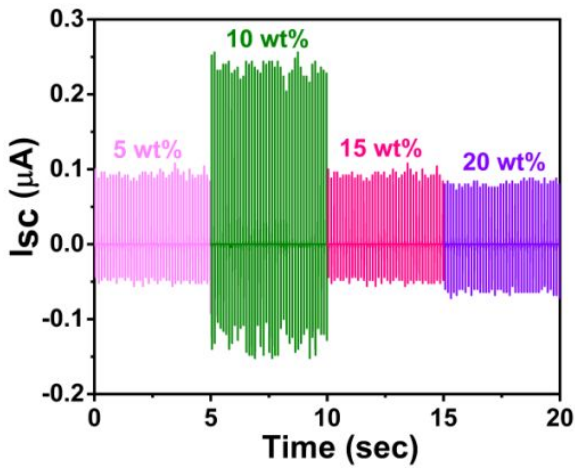

Figure S83. Comparison of output current performance for DPDP.PF $/ \mathrm{TPU}$ composite films in all wt\% composite films 


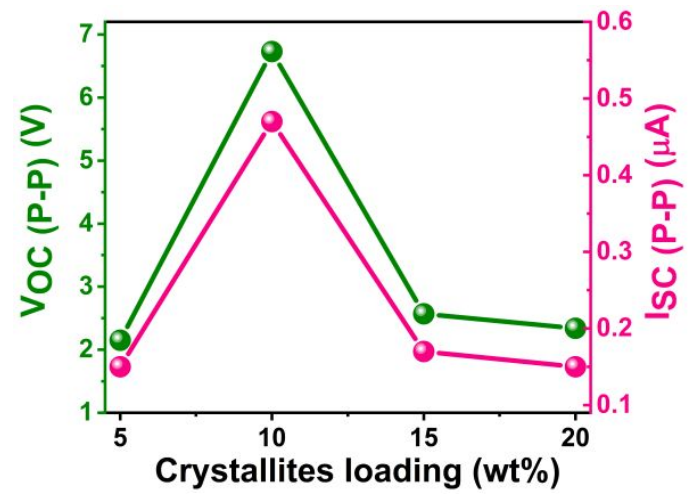

Figure S84. Output voltages and output currents of DPDP.PF ${ }_{6} / T P U$ composite films in all wt $\%$ composite films
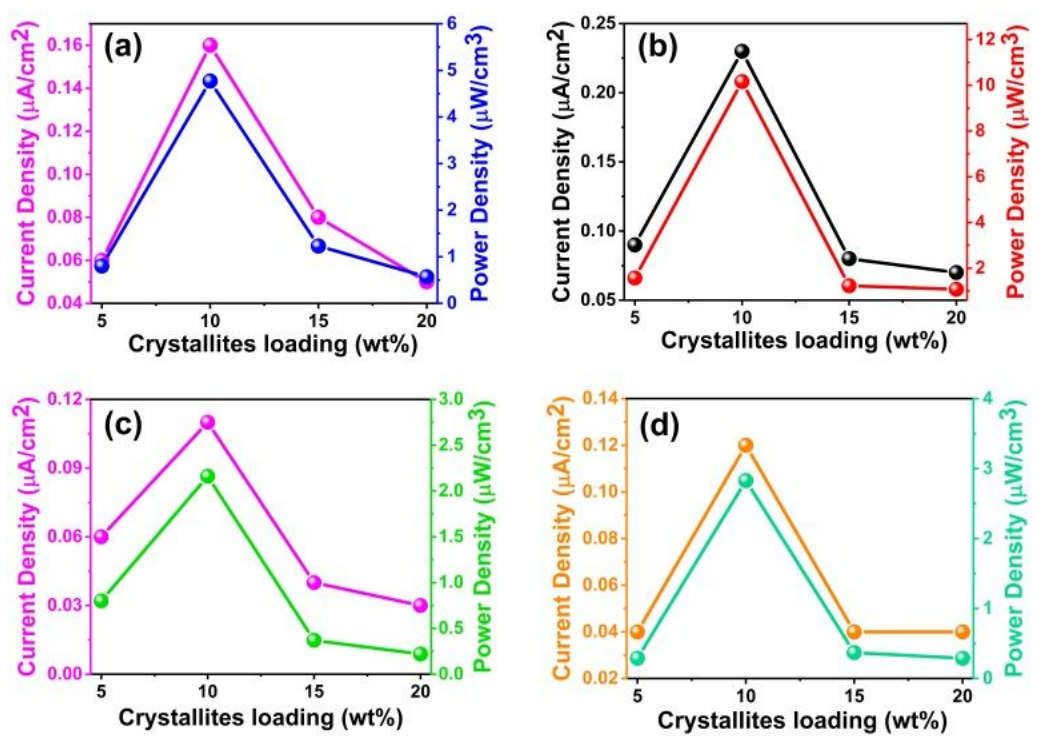

Figure S85. The output current and power density plots for the (a) TPAP·BF 4 /TPU, (b) DPDP.BF $/ \mathrm{TPU}$, (c) TIAP $\cdot \mathrm{BF}_{4} / \mathrm{TPU}$ and (d) DPDP.PF $/$ TPU devices.

Table S10. Table of device output performance of all the wt $\%$ of composites films

\begin{tabular}{|c|c|c|c|c|c|}
\hline $\begin{array}{c}\text { Organic } \\
\text { composites }\end{array}$ & $\begin{array}{c}\text { Composites } \\
\text { (wt\%) }\end{array}$ & $\begin{array}{l}\text { Output voltage } \\
\text { (VPP) }\end{array}$ & $\begin{array}{l}\text { Output current } \\
\text { (IPP) }\end{array}$ & $\begin{array}{l}\text { Power density } \\
\qquad\left(\mu \mathrm{W} / \mathrm{cm}^{3}\right)\end{array}$ & $\begin{array}{l}\text { Current density } \\
\qquad\left(\mu \mathrm{A} / \mathrm{cm}^{2}\right)\end{array}$ \\
\hline TPAP·BF ${ }_{4} / \mathrm{TPU}$ & $\begin{array}{c}5 \\
10 \\
15 \\
20\end{array}$ & $\begin{array}{l}3.34 \\
7.37 \\
3.71 \\
2.98\end{array}$ & $\begin{array}{l}0.25 \\
0.61 \\
0.31 \\
0.21\end{array}$ & $\begin{array}{l}0.80 \\
4.77 \\
1.23 \\
0.57\end{array}$ & $\begin{array}{l}0.06 \\
0.16 \\
0.08 \\
0.05\end{array}$ \\
\hline $\mathrm{DPDP} \cdot \mathrm{BF}_{4} / \mathrm{TPU}$ & $\begin{array}{c}5 \\
10 \\
15 \\
20\end{array}$ & $\begin{array}{l}3.64 \\
8.95 \\
4.55 \\
4.16\end{array}$ & $\begin{array}{l}0.35 \\
0.89 \\
0.31 \\
0.29\end{array}$ & $\begin{array}{c}1.57 \\
10.16 \\
1.23 \\
1.08\end{array}$ & $\begin{array}{l}0.09 \\
0.23 \\
0.08 \\
0.07\end{array}$ \\
\hline TIAP·BF B $_{4} / \mathrm{TPU}$ & $\begin{array}{c}5 \\
10 \\
15 \\
20\end{array}$ & $\begin{array}{l}3.17 \\
4.75 \\
3.26 \\
2.41\end{array}$ & $\begin{array}{l}0.25 \\
0.41 \\
0.17 \\
0.13\end{array}$ & $\begin{array}{l}0.80 \\
2.16 \\
0.37 \\
0.22\end{array}$ & $\begin{array}{l}0.06 \\
0.11 \\
0.04 \\
0.03\end{array}$ \\
\hline DPDP.PF $/ T P U$ & $\begin{array}{c}5 \\
10 \\
15 \\
20\end{array}$ & $\begin{array}{l}2.15 \\
6.73 \\
2.57 \\
2.34\end{array}$ & $\begin{array}{l}0.15 \\
0.47 \\
0.17 \\
0.15\end{array}$ & $\begin{array}{l}0.29 \\
2.83 \\
0.37 \\
0.29\end{array}$ & $\begin{array}{l}0.04 \\
0.12 \\
0.04 \\
0.04 \\
\end{array}$ \\
\hline
\end{tabular}


(a)

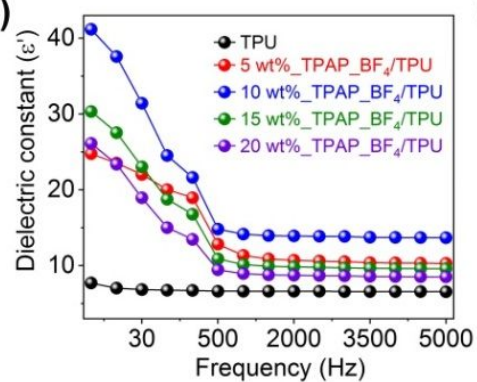

(b)

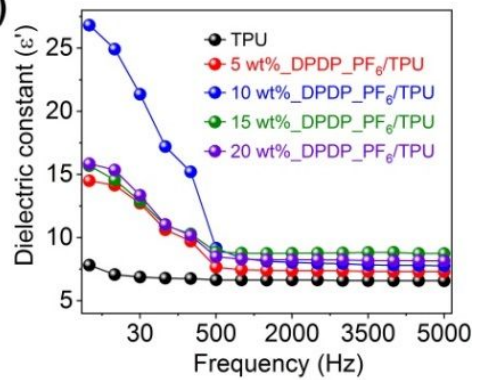

Figure S86. Room temperature $\varepsilon^{\prime}$ data for the composite films of (a) TPAP.BF ${ }_{4} / T P U$ and (b) DPDP.PF 6 /TPU at an operating electric field of $10 \mathrm{~V}$

(a)

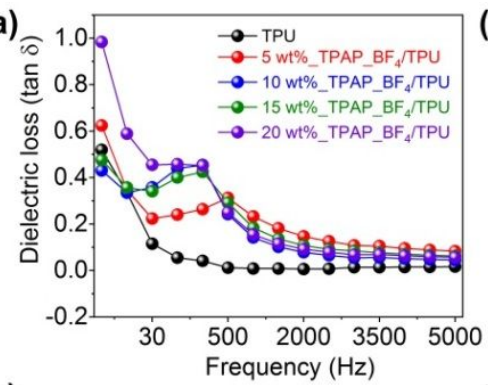

(c)

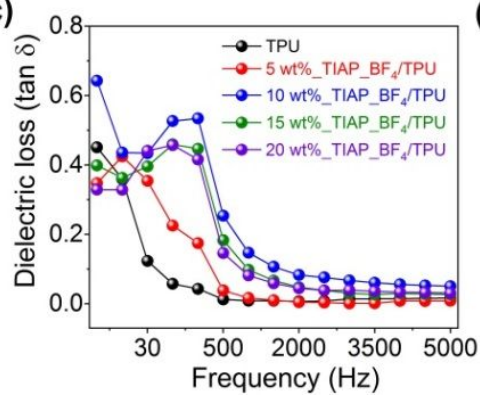

(b)

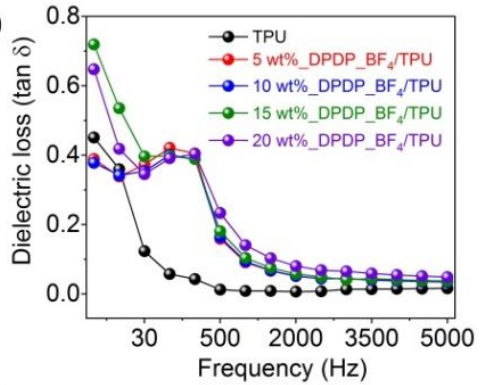

(d)

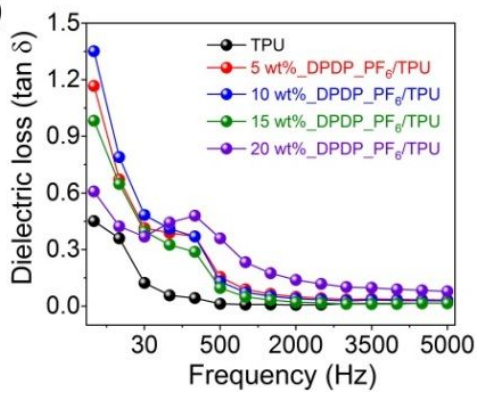

Figure S87. Room temperature tan $\delta$ data for all the composite films

Table S11. The $\varepsilon^{\prime}$ and tan $\delta$ data for all the wt\% composite films at an applied electric field of $10 \mathrm{~V}$ and operating frequency of $1 \mathrm{kHz}$

\begin{tabular}{|c|c|c|c|c|c|c|}
\hline $\begin{array}{l}\text { TPU and composite } \\
\text { materials }\end{array}$ & $\begin{array}{c}\text { Composites } \\
(w t \%)\end{array}$ & $\begin{array}{l}\text { Area } \\
(\mathrm{mm} 2)\end{array}$ & $\begin{array}{c}\text { Thickness } \\
\text { (nm) }\end{array}$ & $\begin{array}{l}\text { Capacitance } \\
\text { (F) }\end{array}$ & $\begin{array}{l}\text { Dielectric } \\
\text { constant }\left(\varepsilon^{\prime}\right)\end{array}$ & $\begin{array}{c}\text { Dielectric } \\
\text { loss (tanס) }\end{array}$ \\
\hline TPU & 0 & 25.57 & $3.1 \mathrm{e} 5$ & $4.85 \mathrm{e}-12$ & 6.64 & 0.0087 \\
\hline \multirow{4}{*}{ TPAP·BF ${ }_{4} / \mathrm{TPU}$} & 5 & 17.01 & $4.0 \mathrm{e} 5$ & $4.28 \mathrm{e}-12$ & 11.36 & 0.2327 \\
\hline & 10 & 11.88 & $4.0 \mathrm{e} 5$ & $3.72 \mathrm{e}-12$ & 14.15 & 0.1419 \\
\hline & 15 & 20.13 & $4.1 \mathrm{e} 5$ & $4.42 \mathrm{e}-12$ & 10.15 & 0.1842 \\
\hline & 20 & 23.66 & $4.1 \mathrm{e} 5$ & $4.57 e-12$ & 8.95 & 0.1551 \\
\hline \multirow{4}{*}{ DPDP. $\mathrm{BF}_{4} / \mathrm{TPU}$} & 5 & 18.79 & $3.1 \mathrm{e} 5$ & $4.44 \mathrm{e}-12$ & 8.27 & 0.0910 \\
\hline & 10 & 20.63 & $4.7 e 5$ & $3.96 e-12$ & 11.72 & 0.0937 \\
\hline & 15 & 21.75 & $5.7 e 5$ & $4.27 e-12$ & 10.99 & 0.1041 \\
\hline & 20 & 20.68 & $3.6 \mathrm{e} 5$ & $4.43 e-12$ & 8.71 & 0.1406 \\
\hline \multirow{4}{*}{ TIAP-BF ${ }_{4} / \mathrm{TPU}$} & 5 & 19.35 & $4.0 \mathrm{e} 5$ & $3.60 \mathrm{e}-12$ & 8.41 & 0.0167 \\
\hline & 10 & 22.02 & $4.8 \mathrm{e} 5$ & $4.56 e-12$ & 11.22 & 0.1472 \\
\hline & 15 & 18.14 & $5.1 \mathrm{e} 5$ & $3.20 \mathrm{e}-12$ & 10.15 & 0.0981 \\
\hline & 20 & 29.05 & $5.0 \mathrm{e} 5$ & $4.57 e-12$ & 8.88 & 0.0818 \\
\hline \multirow{4}{*}{ DPDP.PF 6 /TPU } & 5 & 22.08 & $3.4 \mathrm{e} 5$ & $4.29 e-12$ & 7.47 & 0.0889 \\
\hline & 10 & 16.56 & $3.0 \mathrm{e} 5$ & $4.05 \mathrm{e}-12$ & 8.38 & 0.0731 \\
\hline & 15 & 19.46 & $4.8 \mathrm{e} 5$ & $3.15 e-12$ & 8.77 & 0.0494 \\
\hline & 20 & 22.39 & 4.1e5 & $4.05 e-12$ & 8.29 & 0.2329 \\
\hline
\end{tabular}



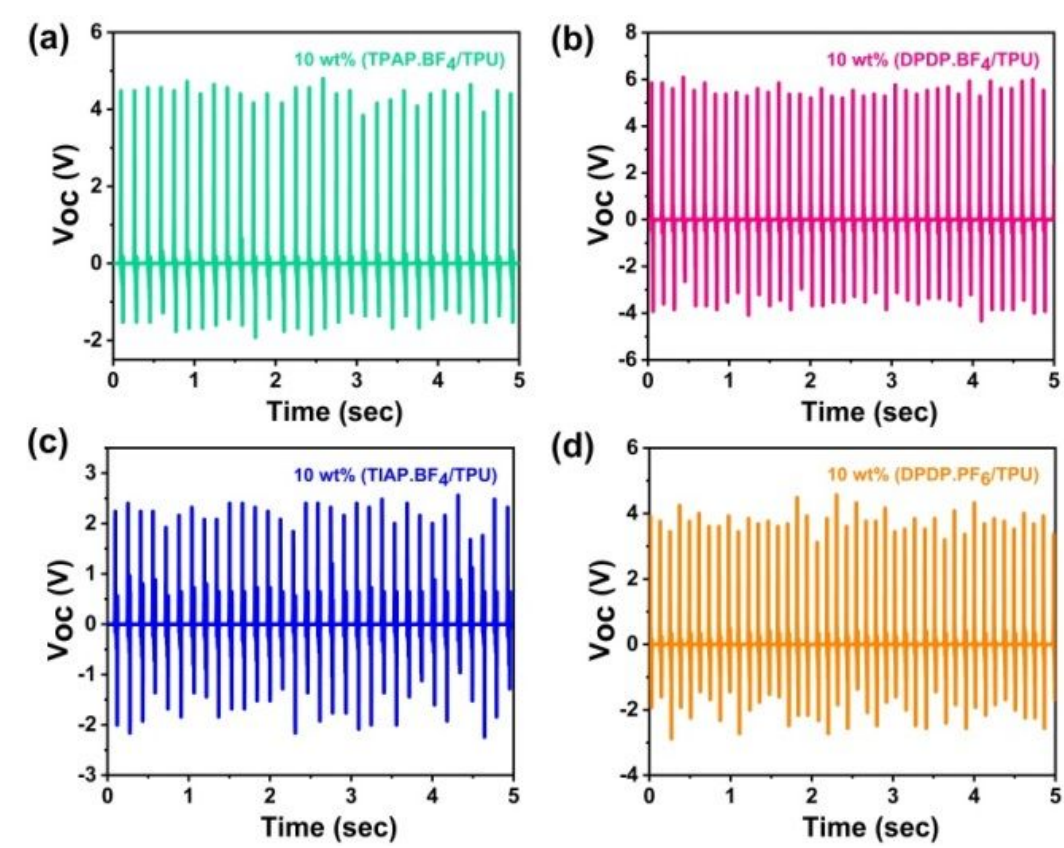

Figure S88. Durability test (after 6 months) for composites (10 wt\%) of (a) TPAP·BF $/$ TPU, (b) DPDP·BF $/$ TPU, (c) $\mathrm{TIAP} \cdot \mathrm{BF}_{4} / \mathrm{TPU}$ and (d) DPDP.PF$/ \mathrm{TPU}$

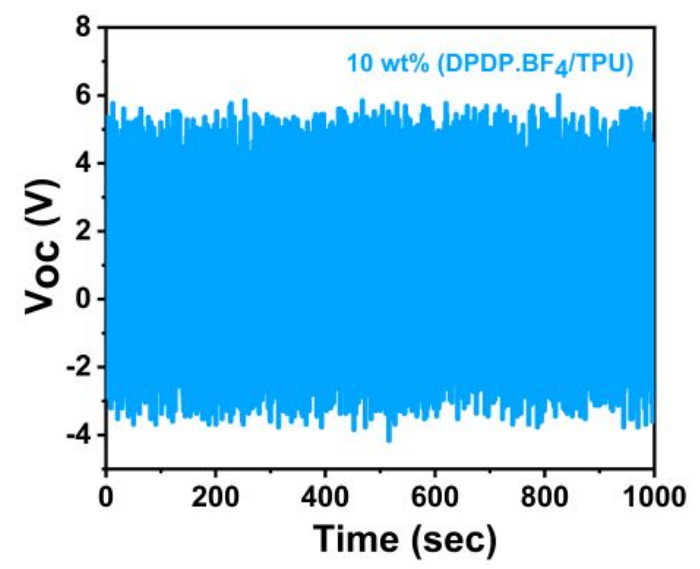

Figure S89. Fatigue test for the $10 \mathrm{wt} \% \mathrm{DPDP} \cdot \mathrm{BF}_{4} / \mathrm{TPU}$ composites with a continuous applied frequency of $8 \mathrm{~Hz}$, over a period of 8000 cycles.

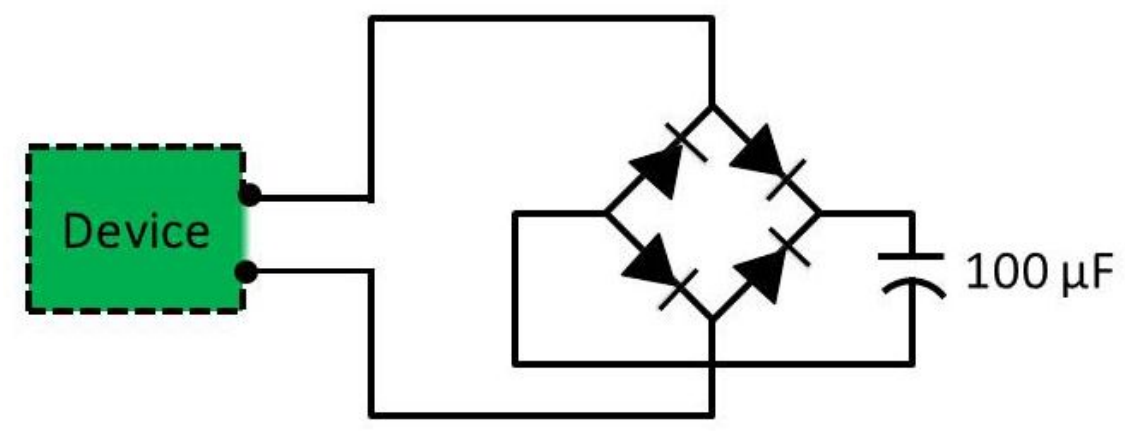

Figure S90. Capacitor charging circuit of full wave-bridge rectifier 

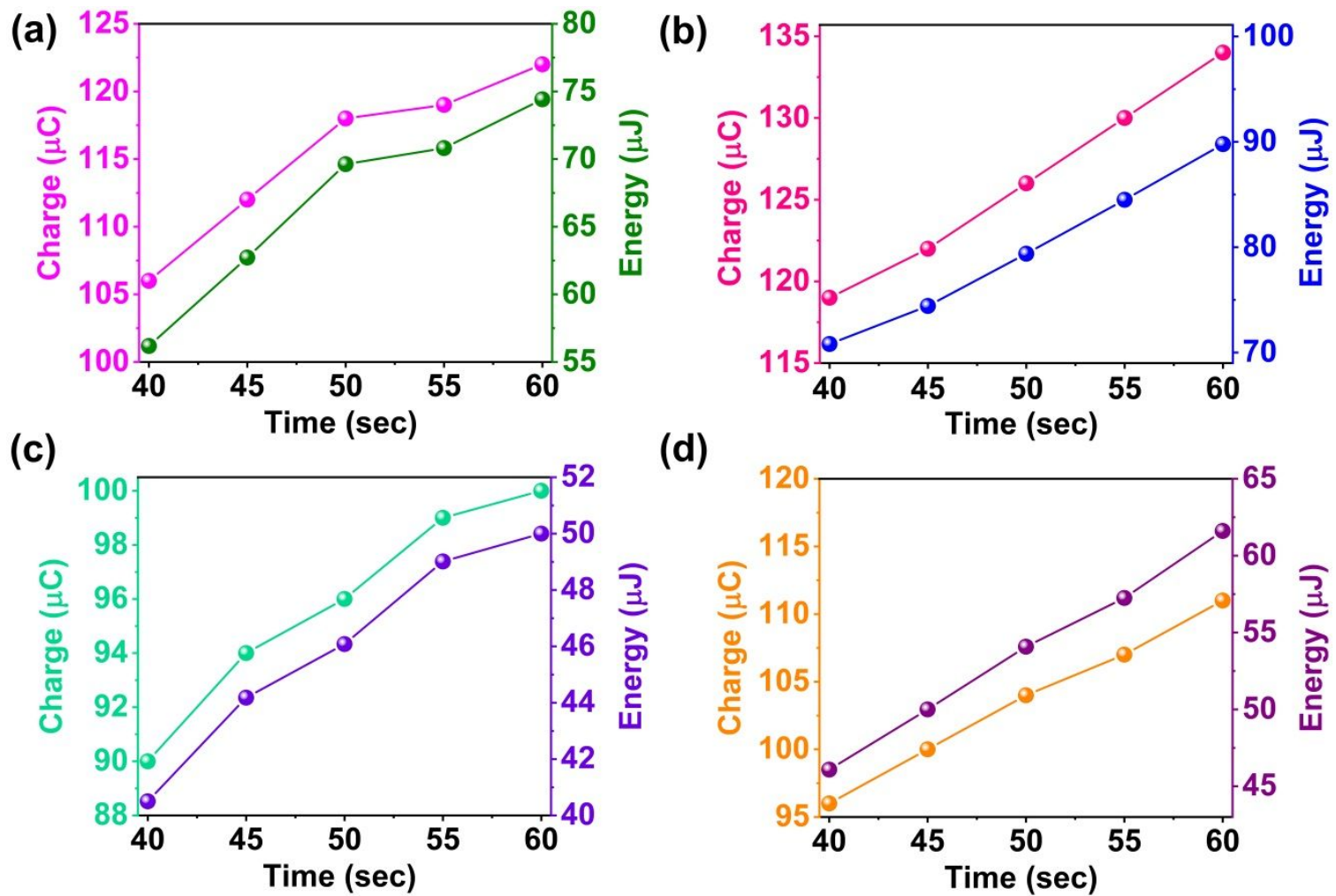

(d)

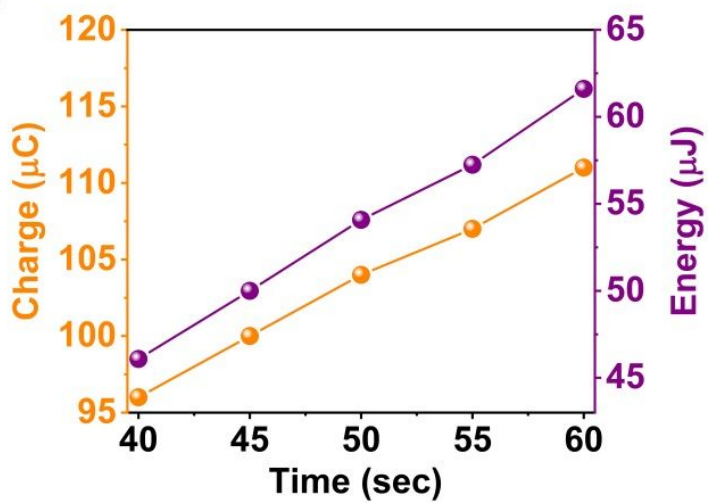

Figure S91. The stored charges $(Q)$ and energies $(E)$ for the $10 \mathrm{wt} \%$ composite capacitors of $(a) T P A P \cdot B F_{4} / T P U,(b)$

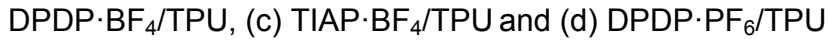

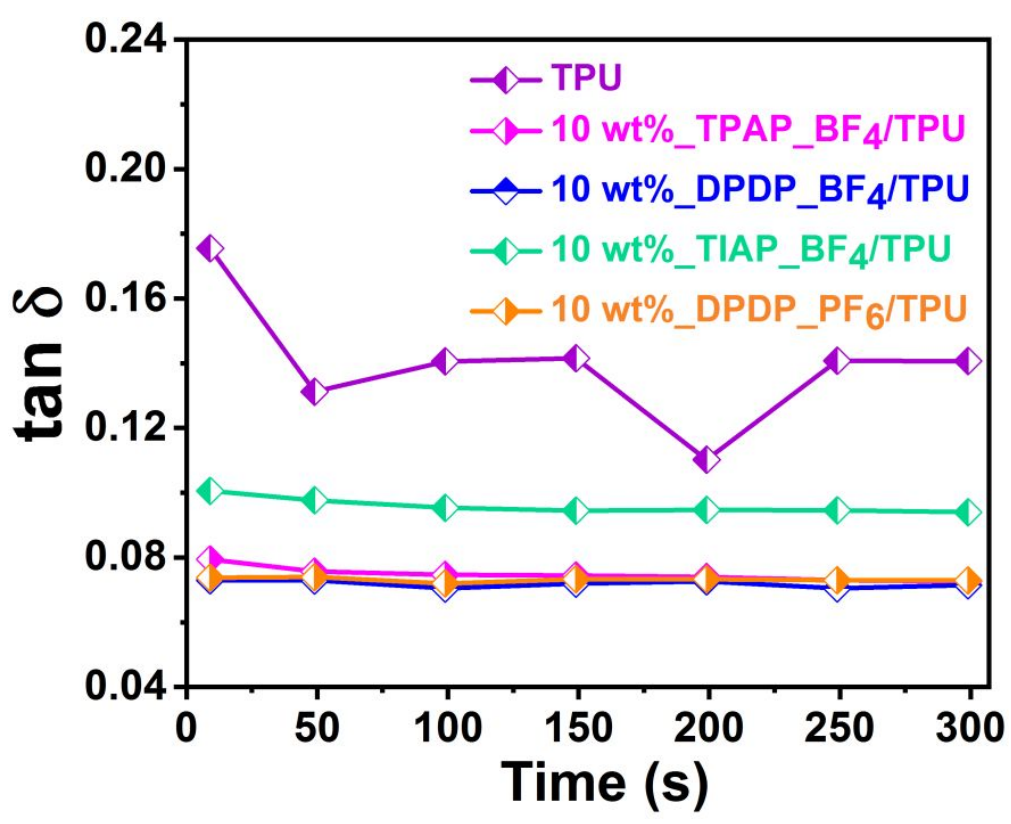

Figure S92. The damping behavior of the neat TPU and $10 \mathrm{wt} \%$ composite films of TPAP.BF $/$ /TPU, DPDP.BF $4 / T P U$, $\mathrm{TIAP} \cdot \mathrm{BF}_{4} / \mathrm{TPU}$ and DPDP.PF$/ \mathrm{TPU}$ 


\section{References}

(1) Gupta, A. K.; Nicholls, J.; Debnath, S.; Rosbottom, I.; Steiner, A.; Boomishankar, R. Cryst. Growth Des. 2011, 11, 555-564.

(2) Zhang, Y.; Ye, H. Y.; Cai, H. L.; Fu, D. W.; Ye, Q.; Zhang, W.; Zhou, Q.; Wang, J.; Yuan, G.L.; Xiong, R. G. Adv. Mater. 2014, 26, 4515-4520.

(3) Horiuchi, S.; Tsutsumi, J.; Kobayashi, K.; Kumai, R.; Ishibashi, S. J. Mater. Chem. C 2018, 6, 4714-4719.

(4) Pan, Q.; Liu, Z. B.; Zhang, H. Y.; Zhang, W. Y.; Tang, Y. Y.; You, Y. M.; Li, P. F.; Liao, W. Q.; Shi, P. P.; Ma, R. W.; Wei, R. Y.; Xiong, R. G. Adv. Mater. 2017, 29, 1700831.

(5) You, Y. M.; Liao, W. Q.; Zhao, D.; Ye, H. Y.; Zhang, Y.; Zhou, Q.; Niu, X.; Wang, J.; Li, P. F.; Fu, D. W.; Wang, Z.; Gao, S.; Yang, K.; Liu, J.; Li, J.; Yan, Y.; Xiong, R. G. Science 2017, 357, 306.

(6) Sun, Z.; Zeb, A.; Liu, S.; Ji, C.; Khan, T.; Li, L.; Hong, M.; Luo, J. Angew. Chem. Int. Ed. 2016, 55, 1185411858. 